\title{
Holomorphically finitely generated algebras
}

\begin{abstract}
Alexei Yu. Pirkovskii*
Abstract. We introduce and study holomorphically finitely generated (HFG) Fréchet algebras, which are analytic counterparts of affine (i.e., finitely generated) $\mathbb{C}$-algebras. Using a theorem of $\mathrm{O}$. Forster, we prove that the category of commutative HFG algebras is anti-equivalent to the category of Stein spaces of finite embedding dimension. We also show that the class of HFG algebras is stable under some natural constructions. This enables us to give a series of concrete examples of HFG algebras, including Arens-Michael envelopes of affine algebras (such as the algebras of holomorphic functions on the quantum affine space and on the quantum torus), the algebras of holomorphic functions on the free polydisk, on the quantum polydisk, and on the quantum polyannulus.
\end{abstract}

Mathematics Subject Classification (2010). 58B34, 32A38, 46H30, 14A22, 16S38, 16S10, $16 \mathrm{~S} 40$.

Keywords. Fréchet algebra, HFG algebra, Stein space, Forster's theorem, smash product, free polydisk, quantum polydisk.

\section{Introduction}

The present paper is motivated by two classical results that underlie noncommutative geometry. The first result, which is essentially a categorical consequence of Hilbert's Nullstellensatz, states that the category of affine algebraic varieties over $\mathbb{C}$ is anti-equivalent to the category of finitely generated commutative unital $\mathbb{C}$ algebras without nilpotents. Explicitly, the anti-equivalence is given by sending an affine variety $X$ to the algebra $\mathscr{O}^{\text {reg }}(X)$ of regular functions on $X$. The second result of the same nature is the Gelfand-Naimark theorem, which establishes an anti-equivalence between the category of locally compact Hausdorff topological spaces and the category of commutative $C^{*}$-algebras by sending each locally compact space $X$ to the algebra $C_{0}(X)$ of continuous functions on $X$ vanishing at infinity. Both results are traditionally viewed as starting points for noncommutative geometry and are often mentioned in introductory textbooks on the subject (see, e.g., $[3,15,22,29,43,48])$.

\footnotetext{
*This work was supported by the RFBR grants no. 12-01-00577 and 15-01-08392.
} 
Our first goal here is to prove a complex analytic analog of the above results. In fact, a significant step in this direction has already been taken by O. Forster [13]. In order to formulate his result, recall (see, e.g., [17]) that for each complex space $\left(X, \mathscr{O}_{X}\right)$ the algebra $\mathscr{O}(X)$ has a canonical topology making it into a Fréchet algebra. If $X$ is reduced (e.g., if $X$ is a complex manifold), then the elements of $\mathscr{O}(X)$ are holomorphic functions on $X$, and the canonical topology on $\mathscr{O}(X)$ is the topology of compact convergence. By definition, a Fréchet algebra $A$ is a Stein algebra if $A$ is topologically isomorphic to $\mathscr{O}(X)$ for some Stein space $\left(X, \mathscr{O}_{X}\right)$.

Theorem 1.1 (O. Forster [13]). The functor $\left(X, \mathscr{O}_{X}\right) \mapsto \mathscr{O}(X)$ is an antiequivalence between the category of Stein spaces and the category of Stein algebras.

Forster's theorem, although somewhat similar to the Nullstellensatz and to the Gelfand-Naimark theorem, still lacks an important feature of the above results. Indeed, it does not identify the category of Stein algebras with the "commutative part" of any category of algebras, and so it does not give us any idea of what "noncommutative Stein spaces" could be. Our goal is to propose a possible approach to this problem for the special case of Stein spaces having finite embedding dimension.

In Section 3, we introduce a category of "holomorphically finitely generated" (HFG for short) Fréchet algebras. Such algebras may be viewed as "analytic counterparts" of affine (i.e., finitely generated) $\mathbb{C}$-algebras. We show that a commutative Fréchet algebra is holomorphically finitely generated if and only if it is topologically isomorphic to the algebra $\mathscr{O}(X)$ for a Stein space $\left(X, \mathscr{O}_{X}\right)$ of finite embedding dimension. Together with Forster's theorem, this implies that the category of Stein spaces of finite embedding dimension in anti-equivalent to the category of commutative HFG algebras. We hope that such a "refinement" of Forster's theorem may contribute to the development of noncommutative complex analytic geometry, a field much less investigated than other types of noncommutative geometry (cf. discussion in [1,23]).

In Sections 4-6, we show that the category of HFG algebras is stable under a number of natural constructions. Section 4 is devoted to Arens-Michael free products [4]. We give several explicit constructions for the Arens-Michael free product, and we show that the Arens-Michael free product of finitely many HFG algebras is again an HFG algebra. In Section 5, we consider two analytic versions of the smash product construction, the $\widehat{\otimes}$-smash product [37] and the ArensMichael smash product. The former is more explicit and easier to deal with, while the latter is better suited for the category of Arens-Michael algebras. We give sufficient conditions for the two smash products to coincide, and we show that the Arens-Michael smash product of HFG algebras is an HFG algebra. In view of further applications, we pay a special attention to the case of semigroup actions and semigroup graded Arens-Michael algebras. Algebras of skew holomorphic functions, or Arens-Michael Ore extensions [38], are discussed in Section 6. They 
can be viewed as "analytic counterparts" of algebraic Ore extensions, and are often reduced to Arens-Michael smash products. Given a balanced domain (respectively, a Reinhardt domain) $D \subset \mathbb{C}^{n}$ and an HFG algebra $A$ endowed with an equicontinuous action $\sigma$ of $\mathbb{Z}_{+}$(respectively, $\left.\mathbb{Z}^{n}\right)$, we define the algebra $\mathscr{O}(D, A ; \sigma)$ of skew $A$ valued holomorphic functions on $D$, and we show that $\mathscr{O}(D, A ; \sigma)$ is an HFG algebra.

Section 7 is entirely devoted to examples of HFG algebras. One source of examples comes from the notion of an Arens-Michael envelope [20, 45]. In Subsection 7.1, we observe that the Arens-Michael envelope of any finitely generated algebra is holomorphically finitely generated. As a consequence, the algebras $\mathscr{O}_{\mathbf{q}}\left(\mathbb{C}^{n}\right)$ and $\mathscr{O}_{\mathbf{q}}\left(\left(\mathbb{C}^{\times}\right)^{n}\right)$ of holomorphic functions on the quantum affine space and on the quantum torus [38,39], the algebra of entire functions on a basis of a Lie algebra [9,11], and some other algebras studied in [38] are holomorphically finitely generated. Other examples are specializations of the constructions discussed in Sections 4-6. In Subsection 7.2, we define the HFG algebra $\mathscr{F}\left(\mathbb{D}_{R}^{n}\right)$ of holomorphic functions on the free polydisk, and we give an explicit power series representation of it. Deformed algebras of holomorphic functions on products of balanced and Reinhardt domains are discussed in Subsection 7.3. Such algebras, being special cases of Arens-Michael Ore extensions, are also HFG algebras. Subsections 7.4 and 7.5 are devoted to the algebras $\mathscr{O}_{\mathbf{q}}\left(\mathbb{D}_{R}^{n}\right)$ and $\mathscr{O}_{\mathbf{q}}\left(\mathbb{D}_{r, R}^{n}\right)$ of holomorphic functions on the quantum polydisk and on the quantum polyannulus, respectively. The former is shown to be a quotient of $\mathscr{F}\left(\mathbb{D}_{R}^{n}\right)$, while the latter is an iterated Arens-Michael Ore extension of the algebra of holomorphic functions on the one-dimensional annulus. As a consequence, both $\mathscr{O}_{\mathbf{q}}\left(\mathbb{D}_{R}^{n}\right)$ and $\mathscr{O}_{\mathbf{q}}\left(\mathbb{D}_{r, R}^{n}\right)$ are HFG algebras.

Some of the results of the present paper were announced in [40].

\section{Preliminaries and notation}

We shall work over the field $\mathbb{C}$ of complex numbers. All algebras are assumed to be associative and unital, and all algebra homomorphisms are assumed to be unital (i.e., to preserve identity elements). In particular, a subalgebra of an algebra $A$ is assumed to contain the identity of $A$.

By a topological algebra we mean an algebra $A$ endowed with a topology making $A$ into a topological vector space and such that the multiplication $A \times A \rightarrow A$ is separately continuous. A Fréchet algebra is a complete metrizable locally convex topological algebra (i.e., a topological algebra whose underlying space is a Fréchet space). A locally $m$-convex algebra [31] is a topological algebra $A$ whose topology can be defined by a family of submultiplicative seminorms (i.e., seminorms $\|\cdot\|$ satisfying $\|a b\| \leq\|a\|\|b\|$ for all $a, b \in A$ ). Equivalently, a topological algebra $A$ is locally $m$-convex if and only if there exists a base $\mathscr{U}$ of 0 -neighborhoods in $A$ 
such that each $U \in \mathscr{U}$ is absolutely convex and idempotent (i.e., satisfies $U^{2} \subseteq$ $U$ ). A complete locally $m$-convex algebra is called an Arens-Michael algebra [19]. The semigroup of all continuous endomorphisms of a topological algebra $A$ will be denoted by $\operatorname{End}(A)$, and the group of all topological automorphisms of $A$ will be denoted by $\operatorname{Aut}(A)$.

The completed projective tensor product of locally convex spaces (see, e.g., $[16,19,24])$ will be denoted by $\widehat{\otimes}$. A complete locally convex topological algebra $A$ with jointly continuous multiplication $A \times A \rightarrow A$ is called a $\widehat{\otimes}$-algebra [19]. For example, each Fréchet algebra and each Arens-Michael algebra are $\widehat{\otimes}$-algebras. The multiplication on a $\widehat{\otimes}$-algebra $A$ uniquely extends to a continuous linear map $\mu_{A}: A \widehat{\otimes} A \rightarrow A$ satisfying the usual associativity conditions. In other words, a $\widehat{\otimes}$ algebra is the same thing as an algebra in the tensor category of complete locally convex spaces endowed with the bifunctor $\widehat{\otimes}$. The notions of $\widehat{\otimes}$-coalgebra, $\widehat{\otimes}$ bialgebra, Hopf $\widehat{\otimes}$-algebra, left/right $\widehat{\otimes}$-module over a $\widehat{\otimes}$-algebra, etc. have obvious meanings: roughly, we just replace $\otimes$ by $\widehat{\otimes}$ in the usual algebraic definitions. In fact, such notions can be defined in a straightforward manner in any symmetric tensor category (cf. discussion in [27]). By an Arens-Michael bialgebra we mean a $\widehat{\otimes}$-bialgebra that is locally $m$-convex as a topological algebra. Similarly, if $H$ is a $\widehat{\otimes}$-bialgebra, then by a left $H$ - $\widehat{\otimes}$-module Arens-Michael algebra we mean a left $H$ - $\widehat{\otimes}$-module algebra that is locally $m$-convex as a topological algebra. If $A$ is a $\widehat{\otimes}$-algebra, we let $\eta_{A}: \mathbb{C} \rightarrow A$ denote the unit map taking $\lambda \in \mathbb{C}$ to $\lambda 1 \in A$. If $C$ is a $\widehat{\otimes}$-coalgebra, then the comultiplication (respectively, the counit) on $C$ will be denoted by $\Delta_{C}$ (respectively, $\varepsilon_{C}$ ). Finally, if $H$ is a Hopf $\widehat{\otimes}$-algebra, then the antipode of $H$ will be denoted by $S_{H}$.

Let $A$ be a topological algebra. Recall from [20] (cf. [45]) that the ArensMichael envelope of $A$ is the completion of $A$ with respect to the family of all continuous submultiplicative seminorms on $A$. Thus we have a canonical continuous homomorphism $i_{A}: A \rightarrow \widehat{A}$, and it is easy to show [20,45] that for each ArensMichael algebra $B$ and each continuous homomorphism $\varphi: A \rightarrow B$ there exists a unique continuous homomorphism $\widehat{\varphi}: \widehat{A} \rightarrow B$ such that $\widehat{\varphi} \circ i_{A}=\varphi$. In other words, we have a natural bijection

$$
\operatorname{Hom}_{\text {AM }}(\widehat{A}, B) \cong \operatorname{Hom}_{\text {Topalg }}(A, B) \quad(B \in \mathrm{AM}),
$$

where Topalg is the category of topological algebras and AM $\subset$ Topalg is the full subcategory consisting of Arens-Michael algebras. Moreover, the correspondence $A \mapsto \widehat{A}$ is a functor from Topalg to AM, and this functor is left adjoint to the inclusion AM $\hookrightarrow$ Topalg (see (2.1)). In what follows, the functor $A \mapsto \widehat{A}$ will be called the Arens-Michael functor.

Here is an important special case. Given an algebra $A$, we can always make $A$ into a topological algebra $A_{\text {str }}$ by endowing $A$ with the strongest locally convex topology (i.e., the topology generated by all seminorms on $A$ ). We define the ArensMichael envelope of $A$ to be the Arens-Michael envelope of $A_{\text {str }}$. 
Arens-Michael envelopes were introduced by J. L. Taylor [45] under the name of "completed locally $m$-convex envelopes". Now it is customary to call them "ArensMichael envelopes", following the terminology suggested by A. Ya. Helemskii [20]. For a more detailed study of Arens-Michael envelopes we refer to [7-11,36-38].

Throughout we will use the following multi-index notation. Let $\mathbb{Z}_{+}=\mathbb{N} \cup\{0\}$ denote the set of all nonnegative integers. For each $n \in \mathbb{N}$ and each $d \in \mathbb{Z}_{+}$, let $W_{n, d}=\{1, \ldots, n\}^{d}$, and let $W_{n}=\bigsqcup_{d \in \mathbb{Z}_{+}} W_{n, d}$. Thus a typical element of $W_{n}$ is a $d$-tuple $\alpha=\left(\alpha_{1}, \ldots, \alpha_{d}\right)$ of arbitrary length $d \in \mathbb{Z}_{+}$, where $\alpha_{j} \in\{1, \ldots, n\}$ for all $j$. The only element of $W_{n, 0}$ will be denoted by $*$. For each $\alpha \in W_{n, d} \subset W_{n}$, let $|\alpha|=d$. Given an algebra $A$, an $n$-tuple $a=\left(a_{1}, \ldots, a_{n}\right) \in A^{n}$, and $\alpha=\left(\alpha_{1}, \ldots, \alpha_{d}\right) \in W_{n}$, we let $a_{\alpha}=a_{\alpha_{1}} \cdots a_{\alpha_{d}} \in A$ if $d>0$; it is also convenient to set $a_{*}=1 \in A$. Given $k=\left(k_{1}, \ldots, k_{n}\right) \in \mathbb{Z}_{+}^{n}$, we let $a^{k}=a_{1}^{k_{1}} \cdots a_{n}^{k_{n}}$. If the $a_{i}$ 's are invertible, then $a^{k}$ makes sense for all $k \in \mathbb{Z}^{n}$. As usual, for each $k=\left(k_{1}, \ldots, k_{n}\right) \in \mathbb{Z}^{n}$ we let $|k|=\left|k_{1}\right|+\cdots+\left|k_{n}\right|$.

Let $S$ be a set, and let $\operatorname{Fin}(S)$ denote the collection of all finite subsets of $S$ ordered by inclusion. Suppose that $E$ is a complete locally convex space, and that $\left(x_{s}\right)_{s \in S}$ is a family of elements of $E$. For each $I \in \operatorname{Fin}(S)$, let $x_{I}=\sum_{s \in I} x_{s}$. By definition $[35,1.3 .8]$, the family $\left(x_{s}\right)_{s \in S}$ is summable if the net $\left(x_{I}\right)_{I \in \operatorname{Fin}(S)}$ converges in $E$. The limit of the above net is denoted by $\sum_{s \in S} x_{s}$. The family $\left(x_{s}\right)_{s \in S}$ is absolutely summable $[35,1.4 .1]$ if for each continuous seminorm $\|\cdot\|$ on $E$ we have $\sum_{s \in S}\left\|x_{s}\right\|<\infty$. Each absolutely summable family in $E$ is summable [35, 1.4.5].

Let $\mathscr{O}\left(\mathbb{C}^{n}\right)$ denote the Fréchet algebra of all holomorphic functions on $\mathbb{C}^{n}$. Recall that for each Arens-Michael algebra $A$ and each commuting $n$-tuple $a=$ $\left(a_{1}, \ldots, a_{n}\right) \in A^{n}$ there exists an entire functional calculus, i.e., a unique continuous homomorphism $\gamma_{a}^{\text {hol }}: \mathscr{O}\left(\mathbb{C}^{n}\right) \rightarrow A$ taking the complex coordinates $z_{1}, \ldots, z_{n}$ to $a_{1}, \ldots, a_{n}$, respectively. Explicitly, $\gamma_{a}^{\text {hol }}$ is given by

$$
\gamma_{a}^{\mathrm{hol}}(f)=f(a)=\sum_{k \in \mathbb{Z}_{+}^{n}} c_{k} a^{k} \quad\left(f=\sum_{k \in \mathbb{Z}_{+}^{n}} c_{k} z^{k} \in \mathscr{O}\left(\mathbb{C}^{n}\right)\right) .
$$

In the language of category theory, the above result means that the algebra $\mathscr{O}\left(\mathbb{C}^{n}\right)$ together with the $n$-tuple $\left(z_{1}, \ldots, z_{n}\right) \in \mathscr{O}\left(\mathbb{C}^{n}\right)^{n}$ represents the functor $A \mapsto A^{n}$ acting from the category CommAM of commutative Arens-Michael algebras to the category of sets. Thus we have a natural bijection

$$
\operatorname{Hom}_{\text {CommaM }}\left(\mathscr{O}\left(\mathbb{C}^{n}\right), A\right) \cong A^{n} \quad(A \in \operatorname{CommAM})
$$

Equivalently, we have $\mathbb{C}\left[z_{1}, \ldots, z_{n}\right]^{\wedge} \cong \mathscr{O}\left(\mathbb{C}^{n}\right)$ (cf. [46]).

A noncommutative (or, more exactly, free) version of the entire functional calculus was introduced by J. L. Taylor [46]. Let $F_{n}=\mathbb{C}\left\langle\zeta_{1}, \ldots, \zeta_{n}\right\rangle$ be the free algebra with generators $\zeta_{1}, \ldots, \zeta_{n}$. The set $\left\{\zeta_{\alpha}: \alpha \in W_{n}\right\}$ of all words in $\zeta_{1}, \ldots, \zeta_{n}$ 
is the standard vector space basis of $F_{n}$. The algebra of free entire functions $[46,47]$ is defined by

$$
\mathscr{F}_{n}=\left\{a=\sum_{\alpha \in W_{n}} c_{\alpha} \zeta_{\alpha}:\|a\|_{\rho}=\sum_{\alpha \in W_{n}}\left|c_{\alpha}\right| \rho^{|\alpha|}<\infty \forall \rho>0\right\} .
$$

The topology on $\mathscr{F}_{n}$ is given by the norms $\|\cdot\|_{\rho}(\rho>0)$, and the multiplication is given by concatenation (like on $F_{n}$ ). Each norm $\|\cdot\|_{\rho}$ is easily seen to be submultiplicative, so $\mathscr{F}_{n}$ is a Fréchet-Arens-Michael algebra containing $F_{n}$ as a dense subalgebra. As was observed by D. Luminet [25], $\mathscr{F}_{n}$ is nuclear. Note also that $\mathscr{F}_{1}$ is topologically isomorphic to $\mathscr{O}(\mathbb{C})$.

Taylor [46] observed that for each Arens-Michael algebra $A$ and each $n$-tuple $a=\left(a_{1}, \ldots, a_{n}\right) \in A^{n}$ there exists a free entire functional calculus, i.e., a unique continuous homomorphism $\gamma_{a}^{\text {free }}: \mathscr{F}_{n} \rightarrow A$ taking the free generators $\zeta_{1}, \ldots, \zeta_{n}$ to $a_{1}, \ldots, a_{n}$, respectively. Explicitly, $\gamma_{a}^{\text {free }}$ is given by

$$
\gamma_{a}^{\text {free }}(f)=f(a)=\sum_{\alpha \in W_{n}} c_{\alpha} a_{\alpha} \quad\left(f=\sum_{\alpha \in W_{n}} c_{\alpha} \zeta_{\alpha} \in \mathscr{F}_{n}\right) .
$$

Similarly to the commutative case, the above result means that the algebra $\mathscr{F}_{n}$ together with the $n$-tuple $\left(\zeta_{1}, \ldots, \zeta_{n}\right) \in \mathscr{F}_{n}^{n}$ represents the functor $A \mapsto A^{n}$ acting from the category AM of Arens-Michael algebras to the category of sets. Thus we have a natural bijection

$$
\operatorname{Hom}_{\mathrm{AM}}\left(\mathscr{F}_{n}, A\right) \cong A^{n} \quad(A \in \mathrm{AM})
$$

Equivalently, we have $\widehat{F}_{n} \cong \mathscr{F}_{n}$ (cf. [46]).

Given $n \in \mathbb{N}$ and $R=\left(R_{1}, \ldots, R_{n}\right) \in(0,+\infty]^{n}$, let

$$
\mathbb{D}_{R}^{n}=\left\{z=\left(z_{1}, \ldots, z_{n}\right) \in \mathbb{C}^{n}:\left|z_{i}\right|<R_{i} \forall i=1, \ldots, n\right\}
$$

denote the open polydisk in $\mathbb{C}^{n}$ of polyradius $R$. If $R_{i}=\rho \in(0,+\infty]$ for all $i$, then we write $\mathbb{D}_{\rho}^{n}$ for $\mathbb{D}_{R}^{n}$. In particular, $\mathbb{D}_{\infty}^{n}=\mathbb{C}^{n}$. If $r, R \in[0,+\infty]^{n}$ and $r_{i}<R_{i}$ for all $i$, let

$$
\mathbb{D}_{r, R}^{n}=\left\{z=\left(z_{1}, \ldots, z_{n}\right) \in \mathbb{C}^{n}: r_{i}<\left|z_{i}\right|<R_{i} \forall i=1, \ldots, n\right\}
$$

denote the open polyannulus of polyradii $r$ and $R$. Clearly, $\mathbb{D}_{R}^{n}=\mathbb{D}_{R_{1}}^{1} \times \cdots \times \mathbb{D}_{R_{n}}^{1}$ and $\mathbb{D}_{r, R}^{n}=\mathbb{D}_{r_{1}, R_{1}}^{1} \times \cdots \times \mathbb{D}_{r_{n}, R_{n}}^{1}$.

The structure sheaf of a complex space $X$ will always be denoted by $\mathscr{O}_{X}$, while the structure sheaf of a scheme $S$ will be denoted by $\mathscr{O}_{S}^{\text {reg }}$. If $X$ is a complex manifold and $E$ is a complete locally convex space, then $\mathscr{O}(X, E)$ stands for the space of all holomorphic $E$-valued functions on $X$. Recall (see, e.g., [16]) that there exists a topological isomorphism $\mathscr{O}(X) \widehat{\otimes} E \cong \mathscr{O}(X, E)$ taking each $f \otimes u \in \mathscr{O}(X) \widehat{\otimes} E$ 
to the function $x \mapsto f(x) u$. Recall also (loc. cit.) that, if $X$ and $Y$ are complex manifolds, then we have a topological isomorphism $\mathscr{O}(X) \widehat{\otimes} \mathscr{O}(Y) \cong \mathscr{O}(X \times Y)$ taking each $f \otimes g \in \mathscr{O}(X) \widehat{\otimes} \mathscr{O}(Y)$ to the function $(x, y) \mapsto f(x) g(y)$.

The completion of a locally convex space $E$ will be denoted by $E^{\sim}$.

\section{HFG algebras and a refinement of Forster's theorem}

We start this section with some elementary observations on the algebra $\mathscr{F}_{n}$. Letting $A=\mathscr{O}\left(\mathbb{C}^{n}\right)$ in $(2.5)$, we obtain a continuous homomorphism

$$
\pi_{n}: \mathscr{F}_{n} \rightarrow \mathscr{O}\left(\mathbb{C}^{n}\right), \quad \zeta_{i} \mapsto z_{i} \quad(i=1, \ldots, n) .
$$

The following is immediate from the universal properties of $\mathscr{O}\left(\mathbb{C}^{n}\right)$ and $\mathscr{F}_{n}$.

Proposition 3.1. For each commutative Arens-Michael algebra A and each $a \in A^{n}$ we have a commutative diagram<smiles>C[C@@H]1[CH][CH]C1</smiles>

Given a topological algebra $A$, let $\overline{[A, A]}$ denote the closure of the two-sided ideal of $A$ generated by the commutators $[a, b]=a b-b a(a, b \in A)$.

Proposition 3.2. The map $\pi_{n}$ given by (3.1) induces a topological algebra isomorphism

$$
\mathscr{F}_{n} / \overline{\left[\mathscr{F}_{n}, \mathscr{F}_{n}\right]} \stackrel{\sim}{\longrightarrow} \mathscr{O}\left(\mathbb{C}^{n}\right), \quad \bar{\zeta}_{i}=\zeta_{i}+\overline{\left[\mathscr{F}_{n}, \mathscr{F}_{n}\right]} \mapsto z_{i} \quad(i=1, \ldots, n) .
$$

Proof. Each continuous homomorphism from $\mathscr{F}_{n}$ to a commutative Arens-Michael

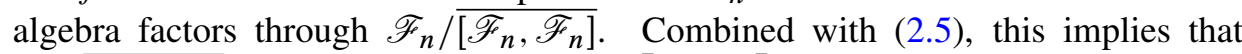
$\mathscr{F}_{n} / \overline{\left[\mathscr{F}_{n}, \mathscr{F}_{n}\right]}$ together with the $n$-tuple $\left(\bar{\zeta}_{1}, \ldots, \bar{\zeta}_{n}\right)$ has the same universal property (2.2) as $\mathscr{O}\left(\mathbb{C}^{n}\right)$. The rest is clear.

Let $A$ and $B$ be Arens-Michael algebras, and let $\varphi: A \rightarrow B$ be a continuous homomorphism. Given $a=\left(a_{1}, \ldots, a_{n}\right) \in A^{n}$, we denote the $n$-tuple $\left(\varphi\left(a_{1}\right), \ldots, \varphi\left(a_{n}\right)\right) \in B^{n}$ simply by $\varphi(a)$.

Proposition 3.3. For each $a \in A^{n}$ and each $f \in \mathscr{F}_{n}$, we have $\varphi(f(a))=f(\varphi(a))$.

Proof. This is immediate either from the power series expansion (2.4) or from the observation that $\varphi \circ \gamma_{a}^{\text {free }}: \mathscr{F}_{n} \rightarrow B, f \mapsto \varphi(f(a))$, is a continuous homomorphism taking each $\zeta_{i}$ to $\varphi\left(a_{i}\right)$. 
Let us introduce some notation. Given an Arens-Michael algebra $A$, an $m$-tuple $a \in A^{m}$, and an $n$-tuple $f=\left(f_{1}, \ldots, f_{n}\right) \in \mathscr{F}_{m}^{n}$, let $f(a)=\left(f_{1}(a), \ldots, f_{n}(a)\right) \in$ $A^{n}$. If now $g \in \mathscr{F}_{n}^{k}$, we define $g \circ f \in \mathscr{F}_{m}^{k}$ by $g \circ f=g(f)$. Thus we obtain a "free superposition" operation

$$
\mathscr{F}_{n}^{k} \times \mathscr{F}_{m}^{n} \rightarrow \mathscr{F}_{m}^{k}, \quad(g, f) \mapsto g \circ f .
$$

Proposition 3.4. For each Arens-Michael algebra A, each $a \in A^{m}$, each $f \in \mathscr{F}_{m}^{n}$, and each $g \in \mathscr{F}_{n}^{k}$ we have $(g \circ f)(a)=g(f(a))$.

Proof. We may assume that $k=1$. Consider the maps $\varphi, \psi: \mathscr{F}_{n} \rightarrow A$ given by

$$
\varphi(g)=g(f(a)), \quad \psi(g)=(g \circ f)(a) \quad\left(g \in \mathscr{F}_{n}\right) .
$$

Clearly, $\varphi=\gamma_{f(a)}^{\mathrm{free}}$ is a continuous homomorphism, and so is $\psi=\gamma_{a}^{\mathrm{free}} \circ \gamma_{f}^{\mathrm{free}}$. For each $i=1, \ldots, n$ we have

$$
\begin{aligned}
\varphi\left(\zeta_{i}\right) & =\zeta_{i}(f(a))=f_{i}(a), \\
\psi\left(\zeta_{i}\right) & =\left(\zeta_{i} \circ f\right)(a)=\left(\zeta_{i}(f)\right)(a)=f_{i}(a) .
\end{aligned}
$$

Therefore $\varphi=\psi$, as required.

Corollary 3.5. The free superposition (3.2) is associative.

Proof. Take $f \in \mathscr{F}_{m}^{n}, g \in \mathscr{F}_{n}^{k}$, and $h \in \mathscr{F}_{k}^{\ell}$. Using Proposition 3.4, we obtain

$$
(h \circ g) \circ f=(h \circ g)(f)=h(g(f))=h \circ(g \circ f) .
$$

From now on, we assume that $A$ is an Arens-Michael algebra.

Definition 3.6. We say that a subalgebra $B \subseteq A$ is holomorphically closed if for each $n \in \mathbb{N}$, each $b \in B^{n}$, and each $f \in \mathscr{F}_{n}$ we have $f(b) \in B$.

In the commutative case, we can use $\mathscr{O}\left(\mathbb{C}^{n}\right)$ instead of $\mathscr{F}_{n}$ :

Proposition 3.7. Let A be a commutative Arens-Michael algebra. Then a subalgebra $B \subseteq A$ is holomorphically closed if and only if for each $n \in \mathbb{N}$, each $b \in B^{n}$, and each $f \in \mathscr{O}\left(\mathbb{C}^{n}\right)$ we have $f(b) \in B$.

Proof. By Propositions 3.1 and 3.2, we have

$$
\left\{f(b): f \in \mathscr{O}\left(\mathbb{C}^{n}\right)\right\}=\left\{g(b): g \in \mathscr{F}_{n}\right\} .
$$

The rest is clear.

Examples 3.8. Of course, if $B$ is closed in $A$, then it is holomorphically closed. More generally, if $B$ is an Arens-Michael algebra under a topology stronger than the topology inherited from $A$, then $B$ is holomorphically closed in $A$. For example, the algebra $C^{\infty}(M)$ of smooth functions on a manifold $M$ is holomorphically closed in $C(M)$. 
Remark 3.9. Our notion of a holomorphically closed subalgebra should not be confused with the more common notion of a subalgebra stable under the holomorphic functional calculus (see, e.g., [3,15]). Recall that a subalgebra $B \subseteq A$ is stable under the holomorphic functional calculus if for each $b \in B$, each neighborhood $U$ of the spectrum $\sigma_{A}(b)$, and each $f \in \mathscr{O}(U)$ we have $f(b) \in B$. Such a subalgebra is necessarily spectrally invariant in $A$, i.e., $\sigma_{B}(b)=\sigma_{A}(b)$ for all $b \in B$. In contrast, a holomorphically closed subalgebra need not be spectrally invariant. For example, for each domain $D \subseteq \mathbb{C}$ the algebra $\mathscr{O}(\mathbb{C})$ may be viewed (via the restriction map) as a holomorphically closed subalgebra of $\mathscr{O}(D)$, but it is not spectrally invariant in $\mathscr{O}(D)$ unless $D=\mathbb{C}$.

It is clear from the definition that the intersection of any family of holomorphically closed subalgebras of $A$ is holomorphically closed. This leads naturally to the following definition.

Definition 3.10. The holomorphic closure, $\operatorname{Hol}(S)$, of a subset $S \subseteq A$ is the intersection of all holomorphically closed subalgebras of $A$ containing $S$.

Clearly, $\operatorname{Hol}(S)$ is the smallest holomorphically closed subalgebra of $A$ containing $S$. It can also be described more explicitly as follows.

Proposition 3.11. For each nonempty subset $S \subseteq A$ we have

$$
\operatorname{Hol}(S)=\left\{f(a): f \in \mathscr{F}_{n}, a \in S^{n}, n \in \mathbb{Z}_{+}\right\} .
$$

Proof. Let $B$ denote the right-hand side of (3.4). Clearly, $S \subseteq B \subseteq \operatorname{Hol}(S)$, so it suffices to show that $B$ is a holomorphically closed subalgebra of $A$. First observe that $1_{A} \in B$ (take any $a \in S$ and $f=1_{\mathscr{F}_{1}}$ ). Let now $a \in S^{m}, b \in S^{n}, f \in \mathscr{F}_{m}$, and $g \in \mathscr{F}_{n}$. We have to show that $f(a)+g(b) \in B$ and $f(a) g(b) \in B$. To this end, define $F, G \in \mathscr{F}_{m+n}$ by

$$
F=f\left(\zeta_{1}, \ldots, \zeta_{m}\right), \quad G=g\left(\zeta_{m+1}, \ldots, \zeta_{m+n}\right),
$$

where the $\zeta_{i}$ 's are now considered as elements of $\mathscr{F}_{m+n}$. We clearly have $f(a)=$ $F(a, b)$ and $g(b)=G(a, b)$. Therefore

$$
f(a)+g(b)=F(a, b)+G(a, b)=(F+G)(a, b) \in B,
$$

and similarly $f(a) g(b) \in B$. Thus $B$ is a subalgebra of $A$.

Let us now show that $B$ is holomorphically closed. Take any $b \in B^{k}$, and represent it in the form

$$
b=\left(f_{1}\left(a^{(1)}\right), \ldots, f_{k}\left(a^{(k)}\right)\right),
$$

where $a^{(i)} \in S^{n_{i}}$ and $f_{i} \in \mathscr{F}_{n_{i}}(i=1, \ldots, k)$. Let $n=n_{1}+\cdots+n_{k}$. By replacing $f_{1}, \ldots, f_{k}$ with suitable elements $\bar{f}_{1}, \ldots, \bar{f}_{k}$ of $\mathscr{F}_{n}$ (where $\bar{f}_{1}=f_{1}\left(\zeta_{1}, \ldots, \zeta_{n_{1}}\right.$ ), $\bar{f}_{2}=f_{2}\left(\zeta_{n_{1}+1}, \ldots, \zeta_{n_{1}+n_{2}}\right)$, etc.), we see that $b=\bar{f}(a)$, where

$$
\bar{f}=\left(\bar{f}_{1}, \ldots, \bar{f}_{k}\right) \in \mathscr{F}_{n}^{k}, \quad a=\left(a^{(1)}, \ldots, a^{(k)}\right) \in S^{n} .
$$


Now Proposition 3.4 implies that for each $g \in \mathscr{F}_{k}$ we have $g(b)=g(\bar{f}(a))=$ $(g \circ \bar{f})(a) \in B$. Thus $B$ is holomorphically closed, as required.

Corollary 3.12. If $A$ is commutative, then for each nonempty subset $S \subseteq A$ we have

$$
\operatorname{Hol}(S)=\left\{f(a): f \in \mathscr{O}\left(\mathbb{C}^{n}\right), a \in S^{n}, n \in \mathbb{Z}_{+}\right\} .
$$

Proof. This is immediate from (3.4) and (3.3).

Corollary 3.13. Let $A$ and $B$ be Arens-Michael algebras, and let $\varphi: A \rightarrow B$ be a continuous homomorphism. Then for each $S \subseteq A$ we have $\varphi(\operatorname{Hol}(S))=\operatorname{Hol}(\varphi(S))$. As a consequence, if $A_{0} \subseteq A$ is a holomorphically closed subalgebra, then $\varphi\left(A_{0}\right)$ is holomorphically closed in $B$.

Proof. Apply $\varphi$ to both sides of (3.4) and use Proposition 3.3.

In the case where $S$ is finite, an even more explicit characterization of $\operatorname{Hol}(S)$ is possible.

Proposition 3.14. For each $a=\left(a_{1}, \ldots, a_{n}\right) \in A^{n}$ we have

$$
\operatorname{Hol}\left(\left\{a_{1}, \ldots, a_{n}\right\}\right)=\left\{f(a): f \in \mathscr{F}_{n}\right\} .
$$

Proof. Let $B$ denote the right-hand side of (3.5), and let $S=\left\{a_{1}, \ldots, a_{n}\right\}$. Clearly, $S \subseteq B \subseteq \operatorname{Hol}(S)$, and $B$ is a subalgebra of $A$. So it suffices to show that $B$ is holomorphically closed in $A$. Each element $b \in B^{k}$ has the form $b=f(a)$ for some $f \in \mathscr{F}_{n}^{k}$. Thus for each $g \in \mathscr{F}_{k}$ we have $g(b)=g(f(a))=(g \circ f)(a) \in B$. Thus $B$ is holomorphically closed, as required.

Corollary 3.15. If $A$ is commutative, then for each $a=\left(a_{1}, \ldots, a_{n}\right) \in A^{n}$ we have

$$
\operatorname{Hol}\left(\left\{a_{1}, \ldots, a_{n}\right\}\right)=\left\{f(a): f \in \mathscr{O}\left(\mathbb{C}^{n}\right)\right\} .
$$

Now we are ready to introduce our main objects of study.

Definition 3.16. Let $A$ be a Fréchet-Arens-Michael algebra. We say that $A$ is holomorphically generated by a subset $S \subseteq A$ if $\operatorname{Hol}(S)=A$. We say that $A$ is holomorphically finitely generated (HFG for short) if $A$ is holomorphically generated by a finite subset.

Example 3.17. By Proposition 3.14, $\mathscr{F}_{n}$ is holomorphically generated by $\zeta_{1}, \ldots, \zeta_{n}$. Similarly, Corollary 3.15 shows that $\mathscr{O}\left(\mathbb{C}^{n}\right)$ is holomorphically generated by $z_{1}, \ldots, z_{n}$. Thus $\mathscr{F}_{n}$ and $\mathscr{O}\left(\mathbb{C}^{n}\right)$ are HFG algebras.

Proposition 3.18. If $A$ is an $H F G$ algebra and $I \subseteq A$ is a closed two-sided ideal, then $A / I$ is also an HFG algebra.

Proof. Clear from Corollary 3.13. 
Remark 3.19. A closed subalgebra of an HFG algebra is not necessarily an HFG algebra (see Remark 7.3 below).

HFG algebras can also be characterized as follows.

Proposition 3.20. Let $A$ be a Fréchet-Arens-Michael algebra.

(i) $A$ is holomorphically finitely generated if and only if $A$ is topologically isomorphic to $\mathscr{F}_{n} / I$ for some $n \in \mathbb{Z}_{+}$and for some closed two-sided ideal $I \subseteq \mathscr{F}_{n}$.

(ii) If $A$ is commutative, then $A$ is holomorphically finitely generated if and only if $A$ is topologically isomorphic to $\mathscr{O}\left(\mathbb{C}^{n}\right) /$ I for some $n \in \mathbb{Z}_{+}$and for some closed two-sided ideal $I \subseteq \mathscr{O}\left(\mathbb{C}^{n}\right)$.

Proof. (i) The "if" part is immediate from Example 3.17 and Proposition 3.18. Conversely, if $A$ is an HFG algebra holomorphically generated by $a_{1}, \ldots, a_{n} \in A$, then Proposition 3.14 implies that $\gamma_{a}^{\text {free }}: \mathscr{F}_{n} \rightarrow A$ (where $a=\left(a_{1}, \ldots, a_{n}\right)$ ) is onto. Applying the Open Mapping Theorem, we obtain a topological algebra isomorphism $A \cong \mathscr{F}_{n} / \operatorname{Ker} \gamma_{a}^{\text {free }}$. This completes the proof of (i). The proof of (ii) is similar (use Corollary 3.15 instead of Proposition 3.14).

Corollary 3.21. Each HFG algebra is nuclear.

The following theorem characterizes commutative HFG algebras as algebras of holomorphic functions.

Theorem 3.22. A commutative Fréchet-Arens-Michael algebra is holomorphically finitely generated if and only if it is topologically isomorphic to $\mathscr{O}(X)$ for some Stein space $\left(X, \mathscr{O}_{X}\right)$ of finite embedding dimension.

Proof. By the Remmert-Bishop-Narasimhan-Wiegmann Embedding Theorem [51], each Stein space $\left(X, \mathscr{O}_{X}\right)$ of finite embedding dimension is biholomorphically equivalent to a closed analytic subspace of $\mathbb{C}^{n}$, where $n \in \mathbb{N}$ is large enough. By H. Cartan's Theorem B, the restriction map $\mathscr{O}\left(\mathbb{C}^{n}\right) \rightarrow \mathscr{O}(X)$ is onto. Applying Proposition 3.20 (ii) and using the Open Mapping Theorem, we conclude that $\mathscr{O}(X)$ is an HFG algebra.

Conversely, let $A$ be a commutative HFG algebra. Taking into account Proposition 3.20 (ii), we may assume that $A=\mathscr{O}\left(\mathbb{C}^{n}\right) / I$, where $I \subseteq \mathscr{O}\left(\mathbb{C}^{n}\right)$ is a closed two-sided ideal. By [12, Satz 2], $\mathscr{O}\left(\mathbb{C}^{n}\right) / I \cong \mathscr{O}(X)$ for a certain closed analytic subspace $X$ of $\mathbb{C}^{n}$ (specifically, $X$ is the zero set of $I$, and $\mathscr{O}_{X}=\mathscr{O}_{\mathbb{C}^{n}} / \mathscr{I}$, where $\mathscr{I} \subseteq \mathscr{O}_{\mathbb{C}^{n}}$ is the ideal sheaf generated by $I$ ). Clearly, $X$ has finite embedding dimension.

Combining Theorem 3.22 with Forster's Theorem 1.1, we obtain the following.

Theorem 3.23. The functor $\left(X, \mathscr{O}_{X}\right) \mapsto \mathscr{O}(X)$ is an anti-equivalence between the category of Stein spaces of finite embedding dimension and the category of commutative $H F G$ algebras. 
Theorem 3.23 looks similar to the Gelfand-Naimark Theorem and to the categorical consequence of the Nullstellensatz (see Section 1). Thus HFG algebras may be considered as candidates for "noncommutative Stein spaces of finite embedding dimension". Of course, this naive point of view needs a solid justification, and it is perhaps too early to say whether it will lead to an interesting theory. As a first step towards this goal, we will give some concrete examples of HFG algebras in Section 7, showing thereby that the class of HFG algebras is rather large. To this end, we will need to show that the class of HFG algebras is stable under a number of natural constructions. This will be done in Sections 4-6.

\section{Free products}

Definition 4.1 (cf. [4]). Let $\left(A_{i}\right)_{i \in I}$ be a family of Arens-Michael algebras. The Arens-Michael free product of $\left(A_{i}\right)_{i \in I}$ is the coproduct of $\left(A_{i}\right)_{i \in I}$ in the category of Arens-Michael algebras, i.e., an Arens-Michael algebra $A$ together with a natural isomorphism

$$
\operatorname{Hom}_{\mathrm{AM}}(A, B) \cong \prod_{i \in I} \operatorname{Hom}_{\mathrm{AM}}\left(A_{i}, B\right) \quad(B \in \mathrm{AM})
$$

More explicitly, the Arens-Michael free product of $\left(A_{i}\right)_{i \in I}$ is an Arens-Michael algebra $A$ together with a family $\left(j_{i}: A_{i} \rightarrow A\right)_{i \in I}$ of continuous homomorphisms such that for each Arens-Michael algebra $B$ and each family $\left(\varphi_{i}: A_{i} \rightarrow B\right)_{i \in I}$ of continuous homomorphisms there exists a unique continuous homomorphism $A \rightarrow B$ making the following diagram (for each $i \in I$ ) commute:

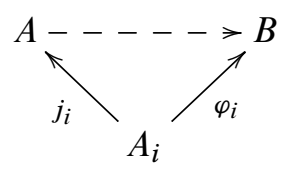

Clearly, if the Arens-Michael free product $A$ of $\left(A_{i}\right)_{i \in I}$ exists, then it is unique up to a unique topological algebra isomorphism over the $A_{i}$ 's. In what follows, we will write $A=\widehat{*}_{i \in I} A_{i}$.

To show that $\widehat{*}_{i \in I} A_{i}$ exists, recall the definition (due to J. Cuntz [4]; cf. also $[38,49,50])$ of an Arens-Michael tensor algebra. Let $E$ be a complete locally convex space, and let $\left\{\|\cdot\|_{\lambda}: \lambda \in \Lambda\right\}$ be a directed defining family of seminorms on $E$. For each $\lambda \in \Lambda$ and each $n \in \mathbb{Z}_{+}$, let $\|\cdot\|_{\lambda}^{(n)}$ denote the $n$th projective tensor power of $\|\cdot\|_{\lambda}$ (we let $\|\cdot\|_{\lambda}^{(0)}=|\cdot|$ by definition). The Arens-Michael tensor algebra (or a 
smooth tensor algebra) $\widehat{T}(E)$ is defined by

$$
\begin{aligned}
\widehat{T}(E)=\left\{a=\sum_{n=0}^{\infty} a_{n}: a_{n} \in E^{\widehat{\otimes} n},\right. & \|a\|_{\lambda, \rho} \\
& \left.=\sum_{n}\left\|a_{n}\right\|_{\lambda}^{(n)} \rho^{n}<\infty \forall \lambda \in \Lambda, \forall \rho>0\right\} .
\end{aligned}
$$

The topology on $\widehat{T}(E)$ is defined by the seminorms $\|\cdot\|_{\lambda, \rho}(\lambda \in \Lambda, \rho>0)$, and the multiplication on $\widehat{T}(E)$ is given by concatenation, like on the usual tensor algebra $T(E)$. Each seminorm $\|\cdot\|_{\lambda, \rho}$ is easily seen to be submultiplicative, and so $\widehat{T}(E)$ is an Arens-Michael algebra containing $T(E)$ as a dense subalgebra. As was observed by Cuntz [4], $\widehat{T}(E)$ has the universal property that, for every Arens-Michael algebra $A$, each continuous linear map $E \rightarrow A$ uniquely extends to a continuous homomorphism $\widehat{T}(E) \rightarrow A$. In other words, there is a natural isomorphism

$$
\operatorname{Hom}_{\mathrm{AM}}(\widehat{T}(E), A) \cong \mathscr{L}(E, A) \quad(A \in \mathrm{AM}),
$$

where $\mathscr{L}(E, A)$ is the space of all continuous linear maps from $E$ to $A$. Note that $\widehat{T}\left(\mathbb{C}^{n}\right) \cong \mathscr{F}_{n}$, and that (2.5) is a special case of (4.2).

Proposition 4.2. Let $\left(A_{i}\right)_{i \in I}$ be a family of Arens-Michael algebras, and let $A$ be the completion of the quotient of $\widehat{T}=\widehat{T}\left(\bigoplus_{i \in I} A_{i}\right)$ by the two-sided closed ideal $J$ generated by all elements of the form

$$
a_{i} \otimes b_{i}-a_{i} b_{i}, \quad 1_{\widehat{T}}-1_{A_{i}} \quad\left(a_{i}, b_{i} \in A_{i}, i \in I\right) .
$$

For each $i \in I$, define $j_{i}: A_{i} \rightarrow A$ to be the composition of the canonical embedding of $A_{i}$ into $\widehat{T}$ with the quotient map $\widehat{T} \rightarrow A$. Then $j_{i}$ is a continuous homomorphism, and $A$ together with $\left(j_{i}: A_{i} \rightarrow A\right)_{i \in I}$ is the Arens-Michael free product of $\left(A_{i}\right)_{i \in I}$.

Proof. Clearly, each $j_{i}$ is a continuous linear map, and it is immediate from the definition of $J$ that $j_{i}$ is an algebra homomorphism. For each Arens-Michael algebra $B$ we have natural isomorphisms

$$
\begin{aligned}
\operatorname{Hom}_{\mathrm{AM}}(A, B) & \cong\left\{\varphi \in \operatorname{Hom}_{\mathrm{AM}}(\widehat{T}, B):\left.\varphi\right|_{J}=0\right\} \\
& \cong\left\{\psi \in \mathscr{L}\left(\bigoplus_{i \in I} A_{i}, B\right):\left.\psi\right|_{A_{i}} \text { is an algebra homomorphism } \forall i \in I\right\} \\
& \cong \prod_{i \in I} \operatorname{Hom}_{\mathrm{AM}}\left(A_{i}, B\right) .
\end{aligned}
$$

A more explicit construction of $\widehat{\star}_{i \in I} A_{i}$ (in the nonunital category, and in the case where $I$ is finite) was given by Cuntz [4]. We need to adapt his construction 
to the unital case, assuming that for each $i \in I$ there exists a closed two-sided ideal $A_{i}^{\circ} \subset A_{i}$ such that $A_{i}=\mathbb{C} 1_{A_{i}} \oplus A_{i}^{\circ}$ as locally convex spaces.

For each $d \geq 2$, let

$$
I_{d}=\left\{\alpha=\left(\alpha_{1}, \ldots, \alpha_{d}\right) \in I^{d}: \alpha_{k} \neq \alpha_{k+1} \forall k=1, \ldots, d-1\right\} .
$$

Let also $I_{1}=I, I_{0}=\{*\}$ (the singleton), and $I_{\infty}=\bigsqcup_{d \in \mathbb{Z}_{+}} I_{d}$. For each $d \in \mathbb{Z}_{+}$ and each $\alpha \in I_{d}$, we let $|\alpha|=d$. Given $d>0$ and $\alpha=\left(\alpha_{1}, \ldots, \alpha_{d}\right) \in I_{\infty}$, let $A_{\alpha}=A_{\alpha_{1}}^{\circ} \widehat{\otimes} \cdots \widehat{\otimes} A_{\alpha_{d}}^{\circ}$. For $d=0$, we let $A_{*}=\mathbb{C}$. For each $i \in I$, choose a directed defining family $\left\{\|\cdot\|_{v}: v \in \Lambda_{i}\right\}$ of submultiplicative seminorms on $A_{i}^{\circ}$. Let $\Lambda=\prod_{i \in I} \Lambda_{i}$, and, for each $\lambda=\left(\lambda_{i}\right)_{i \in I} \in \Lambda$ and each $\alpha=\left(\alpha_{1}, \ldots, \alpha_{d}\right) \in I_{\infty}$, let $\|\cdot\|_{\lambda}^{(\alpha)}$ denote the projective tensor product of the seminorms $\|\cdot\|_{\lambda_{\alpha_{1}}}, \ldots,\|\cdot\|_{\lambda_{\alpha_{k}}}$. For $d=0$, it is convenient to set $\|\cdot\|_{\lambda}^{(*)}=|\cdot|$. Let $P(I)=[1,+\infty)^{I}$ denote the family of all functions on $I$ with values in $[1,+\infty)$. Given $p \in P(I)$, we let $p_{i}=p(i)$ for $i \in I, p_{\alpha}=p_{\alpha_{1}} \cdots p_{\alpha_{d}}$ for $\alpha=\left(\alpha_{1}, \ldots, \alpha_{d}\right) \in I_{\infty}$ with $d>0$, and $p_{*}=1$. Let

$$
\begin{aligned}
A=\left\{a=\sum_{\alpha \in I_{\infty}} a_{\alpha}: a_{\alpha}\right. & \in A_{\alpha},\|a\|_{\lambda, p} \\
& \left.=\sum_{\alpha \in I_{\infty}}\left\|a_{\alpha}\right\|_{\lambda}^{(\alpha)} p_{\alpha}<\infty \forall \lambda \in \Lambda, \forall p \in P(I)\right\} .
\end{aligned}
$$

A standard argument shows that $A$ is a complete locally convex space with respect to the topology determined by the seminorms $\|\cdot\|_{\lambda, p}(\lambda \in \Lambda, p \in P(I))$.

Now, given $\alpha=\left(\alpha_{1}, \ldots, \alpha_{d}\right) \in I_{\infty}$, let $A_{\alpha}^{\text {alg }}=A_{\alpha_{1}}^{\circ} \otimes \cdots \otimes A_{\alpha_{d}}^{\circ}$. The algebraic sum

$$
A^{\mathrm{alg}}=\bigoplus_{\alpha \in I_{\infty}} A_{\alpha}^{\mathrm{alg}}
$$

is clearly a dense subspace of $A$. Recall from [34] that there is a multiplication on $A^{\text {alg }}$ such that each $A_{i}$ becomes a subalgebra of $A^{\text {alg }}$ and such that $A^{\text {alg }}=*_{i \in I} A_{i}$, the algebraic free product of $\left(A_{i}\right)_{i \in I}$. Specifically, the multiplication on $A^{\text {alg }}$ is given by concatenation composed (if necessary) with the product maps $A_{i}^{\circ} \otimes A_{i}^{\circ} \rightarrow$ $A_{i}^{\circ}(i \in I)$.

Proposition 4.3. The multiplication on $A^{\mathrm{alg}}$ uniquely extends to a continuous multiplication on $A$. The resulting algebra A together with the canonical embeddings $A_{i} \rightarrow A$ is the Arens-Michael free product of $\left(A_{i}\right)_{i \in I}$. Moreover, each seminorm $\|\cdot\|_{\lambda, p}(\lambda \in \Lambda, p \in P(I))$ is submultiplicative.

Proof. Let us show that each seminorm $\|\cdot\|_{\lambda, p}$ is submultiplicative on $A^{\text {alg. Take }}$ any $a \in A_{\alpha}^{\mathrm{alg}}$ and $b \in A_{\beta}^{\mathrm{alg}}$, where $\alpha=\left(\alpha_{1}, \ldots, \alpha_{d}\right) \in I_{\infty}, \beta=\left(\beta_{1}, \ldots, \beta_{m}\right) \in I_{\infty}$. 
Suppose first that $\alpha_{d} \neq \beta_{1}$. Then $\gamma=(\alpha, \beta) \in I_{\infty}, a b=a \otimes b \in A_{\alpha}^{\mathrm{alg}} \otimes A_{\beta}^{\mathrm{alg}}=$ $A_{\gamma}^{\mathrm{alg}}$, and

$$
\|a b\|_{\lambda, p}=\|a \otimes b\|_{\lambda}^{(\gamma)} p_{\gamma}=\|a\|_{\lambda}^{(\alpha)}\|b\|_{\lambda}^{(\beta)} p_{\alpha} p_{\beta}=\|a\|_{\lambda, p}\|b\|_{\lambda, p} .
$$

Now consider the case where $\alpha_{d}=\beta_{1}$. Let $\gamma=\left(\alpha_{1}, \ldots, \alpha_{d}, \beta_{2}, \ldots, \beta_{m}\right) \in I_{\infty}$. Denote by $\mu: A_{\alpha_{d}}^{\circ} \otimes A_{\beta_{1}}^{\circ} \rightarrow A_{\alpha_{d}}^{\circ}$ the product map on $A_{\alpha_{d}}^{\circ}$, and let

$$
\bar{\mu}=1 \otimes \mu \otimes 1: A_{\alpha}^{\mathrm{alg}} \otimes A_{\beta}^{\mathrm{alg}} \rightarrow A_{\gamma}^{\mathrm{alg}} .
$$

Then $a b=\bar{\mu}(a \otimes b) \in A_{\gamma}^{\mathrm{alg}}$. Clearly, for each $u \in A_{\alpha}^{\mathrm{alg}} \otimes A_{\beta}^{\mathrm{alg}}$ we have $\|\bar{\mu}(u)\|_{\lambda}^{(\gamma)} \leq$ $\|u\|_{\lambda}^{(\alpha, \beta)}$, where $\|\cdot\|_{\lambda}^{(\alpha, \beta)}$ is the projective tensor product of the seminorms $\|\cdot\|_{\lambda}^{(\alpha)}$ and $\|\cdot\|_{\lambda}^{(\beta)}$. Therefore

$$
\|a b\|_{\lambda, p}=\|\bar{\mu}(a \otimes b)\|_{\lambda}^{(\gamma)} p_{\gamma} \leq\|a\|_{\lambda}^{(\alpha)}\|b\|_{\lambda}^{(\beta)} p_{\alpha} p_{\beta}=\|a\|_{\lambda, p}\|b\|_{\lambda, p} .
$$

Finally, take any $a, b \in A^{\text {alg }}$ and decompose them as

$$
a=\sum_{\alpha \in I_{\infty}} a_{\alpha}, \quad b=\sum_{\beta \in I_{\infty}} b_{\beta} \quad\left(a_{\alpha} \in A_{\alpha}^{\mathrm{alg}}, b_{\beta} \in A_{\beta}^{\mathrm{alg}}\right) .
$$

By using (4.5) and (4.6), we obtain

$$
\|a b\|_{\lambda, p}=\left\|\sum_{\alpha, \beta} a_{\alpha} b_{\beta}\right\|_{\lambda, p} \leq \sum_{\alpha, \beta}\left\|a_{\alpha}\right\|_{\lambda, p}\left\|b_{\beta}\right\|_{\lambda, p}=\|a\|_{\lambda, p}\|b\|_{\lambda, p} .
$$

Thus each seminorm $\|\cdot\|_{\lambda, p}$ is submultiplicative on $A^{\text {alg }}$, and so $A^{\text {alg }}$ is a locally $m$ convex algebra. Since $A$ is the completion of $A^{\text {alg }}$, it follows that the multiplication on $A^{\text {alg }}$ uniquely extends to a continuous multiplication on $A$ making $A$ into an Arens-Michael algebra. Moreover, each seminorm $\|\cdot\|_{\lambda, p}$ is submultiplicative on A.

Let now $B$ be an Arens-Michael algebra, and let $\left(\varphi_{i}: A_{i} \rightarrow B\right)_{i \in I}$ be a family of continuous homomorphisms. Since $A^{\text {alg }}=*_{i \in I} A_{i}$, there exists a unique algebra homomorphism $\varphi^{\text {alg: }} A^{\text {alg }} \rightarrow B$ such that $\left.\varphi^{\text {alg }}\right|_{A_{i}}=\varphi_{i}$ for all $i \in I$. We claim that $\varphi^{\text {alg }}$ is continuous with respect to the topology inherited from $A$. Indeed, let $\|\cdot\|$ be a continuous submultiplicative seminorm on $B$. Then for each $i \in I$ there exists $p_{i} \geq 1$ and $\lambda_{i} \in \Lambda_{i}$ such that for each $a \in A_{i}^{\circ}$ we have

$$
\left\|\varphi_{i}(a)\right\| \leq p_{i}\|a\|_{\lambda_{i}} .
$$

Given $\alpha=\left(\alpha_{1}, \ldots, \alpha_{d}\right) \in I_{\infty}$, let $\varphi_{\alpha}^{\text {alg }}=\left.\varphi^{\text {alg }}\right|_{A_{\alpha}^{\text {alg }}}$. By construction (cf. [34]), $\varphi_{\alpha}^{\text {alg }}$ is the composition of the maps

$$
A_{\alpha_{1}}^{\circ} \otimes \cdots \otimes A_{\alpha_{d}}^{\circ} \stackrel{\varphi_{\alpha_{1}} \otimes \cdots \otimes \varphi_{\alpha_{d}}}{\longrightarrow} B \otimes \cdots \otimes B \stackrel{\mu_{d}}{\longrightarrow} B,
$$


where $\mu_{d}$ is the product map. Now it follows from (4.7) and from the submultiplicativity of $\|\cdot\|$ that for each $a \in A_{\alpha}^{\text {alg }}$ we have

$$
\left\|\varphi_{\alpha}^{\mathrm{alg}}(a)\right\| \leq p_{\alpha}\|a\|_{\lambda}^{(\alpha)},
$$

where $\lambda=\left(\lambda_{i}\right)_{i \in I} \in \Lambda$ and $p=\left(p_{i}\right)_{i \in I} \in P(I)$. Finally, for each $a=$ $\sum_{|\alpha|>0} a_{\alpha} \in A^{\text {alg }}$ (where $a_{\alpha} \in A_{\alpha}^{\text {alg }}$ ) we obtain

$$
\left\|\varphi^{\mathrm{alg}}\left(\sum_{\alpha} a_{\alpha}\right)\right\| \leq \sum_{\alpha}\left\|\varphi_{\alpha}^{\mathrm{alg}}\left(a_{\alpha}\right)\right\| \leq \sum_{\alpha}\left\|a_{\alpha}\right\|_{\lambda}^{(\alpha)} p_{\alpha}=\|a\|_{\lambda, p} .
$$

Therefore $\varphi^{\text {alg }}$ is continuous, and so it uniquely extends to a continuous homomorphism $\varphi: A \rightarrow B$. Thus $A=\widehat{*}_{i \in I} A_{i}$, as required.

Corollary 4.4. Under the above assumptions, suppose that I is finite. Then

$$
\begin{aligned}
\underset{i \in I}{\widehat{*}} A_{i}=\left\{a=\sum_{\alpha \in I_{\infty}} a_{\alpha}: a_{\alpha}\right. & \in A_{\alpha},\|a\|_{\lambda, \tau} \\
& \left.=\sum_{\alpha \in I_{\infty}}\left\|a_{\alpha}\right\|_{\lambda}^{(\alpha)} \tau^{|\alpha|}<\infty \forall \lambda \in \Lambda, \forall \tau \geq 1\right\} .
\end{aligned}
$$

Moreover, each seminorm $\|\cdot\|_{\lambda, \tau}(\lambda \in \Lambda, \tau \geq 1)$ is submultiplicative.

Proof. For each $\tau \geq 1$ we have $\|\cdot\|_{\lambda, \tau}=\|\cdot\|_{\lambda, p}$, where $p_{i}=\tau$ for all $i \in I$. Conversely, for each $p \in P(I)$ we have $\|\cdot\|_{\lambda, p} \leq\|\cdot\|_{\lambda, \tau}$, where $\tau=\max _{i \in I} p_{i}$. Now (4.8) follows from (4.4) and Proposition 4.3.

Here is a simple example.

Proposition 4.5. For each family $\left(E_{i}\right)_{i \in I}$ of complete locally convex spaces there exists a topological algebra isomorphism

$$
\underset{i \in I}{\widehat{*}} \widehat{T}\left(E_{i}\right) \cong \widehat{T}\left(\bigoplus_{i \in I} E_{i}\right)
$$

In particular,

$$
\begin{aligned}
& \mathscr{F}_{m} \widehat{*} \mathscr{F}_{n} \cong \mathscr{F}_{m+n}, \\
& \mathscr{F}_{n} \cong \mathscr{O}(\mathbb{C}) \widehat{*} \cdots \widehat{*} \mathscr{O}(\mathbb{C}) \quad(\text { f factors }) .
\end{aligned}
$$

Proof. This is immediate from (4.1), from (4.2), and from the fact that the locally convex direct sum is the coproduct in the category of locally convex spaces.

The next observation shows that the Arens-Michael free product commutes with quotients (modulo completions). 
Proposition 4.6. Let $\left(A_{i}\right)_{i \in I}$ be a family of Arens-Michael algebras, and let, for each $i \in I, J_{i}$ be a closed two-sided ideal of $A_{i}$. Denote by $J$ the closed twosided ideal of $\widehat{*}_{i \in I} A_{i}$ generated by all the $J_{i}$ 's. There exists a topological algebra isomorphism

$$
\underset{i \in I}{\widehat{*}}\left(\left(A_{i} / J_{i}\right)^{\sim}\right) \cong\left(\left(\underset{i \in I}{\widehat{*}} A_{i}\right) / J\right)^{\sim} .
$$

Proof. For each Arens-Michael algebra $B$ we have natural isomorphisms

$$
\begin{aligned}
\operatorname{Hom}_{\mathrm{AM}}\left(\left(\left(\underset{i \in I}{\widehat{*}} A_{i}\right) / J\right)^{\sim}, B\right) & \cong\left\{\varphi \in \operatorname{Hom}_{\mathrm{AM}}\left(\underset{i \in I}{\widehat{*}} A_{i}, B\right): \varphi(J)=0\right\} \\
& \cong\left\{\varphi \in \operatorname{Hom}_{\mathrm{AM}}\left(\underset{i \in I}{\widehat{*}} A_{i}, B\right): \varphi\left(J_{i}\right)=0 \forall i \in I\right\} \\
& \cong\left\{\varphi=\left(\varphi_{i}\right)_{i \in I} \in \prod_{i \in I} \operatorname{Hom}_{\mathrm{AM}}\left(A_{i}, B\right): \varphi_{i}\left(J_{i}\right)=0 \forall i \in I\right\} \\
& \cong \prod_{i \in I} \operatorname{Hom}_{\mathrm{AM}}\left(\left(A_{i} / J_{i}\right)^{\sim}, B\right) .
\end{aligned}
$$

Corollary 4.7. If $A_{1}$ and $A_{2}$ are $H F G$ algebras, then so is $A_{1} \widehat{*} A_{2}$.

Proof. By Proposition 3.20, each $A_{k}(k=1,2)$ is a quotient of $\mathscr{F}_{n_{k}}$ for some $n_{k} \in \mathbb{N}$. Applying Proposition 4.6 and using (4.9), we see that $A_{1} \widehat{*} A_{2}$ is a quotient of $\mathscr{F}_{n_{1}+n_{2}}$. Hence $A_{1} \widehat{*} A_{2}$ is an HFG algebra by Proposition 3.20.

\section{Smash products}

In this section we consider two analytic versions of the smash product construction (see, e.g., $[5,14,27,44]$ ), the $\widehat{\otimes}$-smash product and the Arens-Michael smash product. While the former is a special case of smash products in tensor categories [27], the latter appears to be new. Let us start by recalling some definitions [26,27].

Let $H$ be a $\widehat{\otimes}$-bialgebra. Similarly to the algebraic case (see, e.g., [21]), the projective tensor product of two left $H$ - $\widehat{\otimes}$-modules (respectively, comodules) has a natural structure of a left $H-\widehat{\otimes}$-module (respectively, comodule). A left $H-\widehat{\otimes}$ module algebra is a $\widehat{\otimes}$-algebra $A$ endowed with the structure of a left $H$ - $\widehat{\otimes}$-module in such a way that the product $\mu_{A}: A \widehat{\otimes} A \rightarrow A$ and the unit map $\eta_{A}: \mathbb{C} \rightarrow A$ are $H$ - $\widehat{\otimes}$-module morphisms. Similarly, a left $H-\widehat{\otimes}$-comodule algebra is a $\widehat{\otimes}$-algebra $B$ endowed with the structure of a left $H-\widehat{\otimes}$-comodule in such a way that the product $\mu_{B}: B \widehat{\otimes} B \rightarrow B$ and the unit map $\eta_{B}: \mathbb{C} \rightarrow B$ are $H$ - $\widehat{\otimes}$-comodule morphisms. Given a left $H$ - $\widehat{\otimes}$-module algebra $A$ and a left $H$ - $\widehat{\otimes}$-comodule algebra $B$, the $\widehat{\otimes}$ smash product $A \widehat{\#}^{H} B$ is defined as follows. As a topological vector space, $A \widehat{\#}^{H} B$ is equal to $A \widehat{\otimes} B$. To define multiplication, denote by $\mu_{H, A}: H \widehat{\otimes} A \rightarrow A$ the 
action of $H$ on $A$, by $\Delta_{H, B}: B \rightarrow H \widehat{\otimes} B$ the coaction of $H$ on $B$, and define $\tau_{B, A}: B \widehat{\otimes} A \rightarrow A \widehat{\otimes} B$ to be the composition

$$
B \widehat{\otimes} A \stackrel{\Delta_{H, B} \otimes 1_{A}}{\longrightarrow} H \widehat{\otimes} B \widehat{\otimes} A \stackrel{1_{H} \otimes c_{B, A}}{\longrightarrow} H \widehat{\otimes} A \widehat{\otimes} B \stackrel{\mu_{H, A} \otimes 1_{B}}{\longrightarrow} A \widehat{\otimes} B
$$

(here $c_{B, A}$ stands for the flip $B \widehat{\otimes} A \rightarrow A \widehat{\otimes} B$ ). Then the map

$$
(A \widehat{\otimes} B) \widehat{\otimes}(A \widehat{\otimes} B) \stackrel{1_{A} \otimes \tau_{B, A} \otimes 1_{B}}{\longrightarrow} A \widehat{\otimes} A \widehat{\otimes} B \widehat{\otimes} B \stackrel{\mu_{A} \otimes \mu_{B}}{\longrightarrow} A \widehat{\otimes} B
$$

is an associative multiplication on $A \widehat{\otimes} B$ (the associativity was proved in [26] for the special case $B=H$; the proof extends verbatim to an arbitrary $B$ ). The resulting $\widehat{\otimes}$-algebra is denoted by $A \widehat{\#}^{H} B$ and is called the $\widehat{\otimes}$-smash product of $A$ and $B$ over $H$.

To motivate our further constructions, let us show that $A \widehat{\#}^{H} B$ is characterized by a universal property. We need a simple lemma.

Lemma 5.1. Let $H$ be a $\widehat{\otimes}$-bialgebra, $A$ be a left $H-\widehat{\otimes}$-module algebra, and $B$ be a left $H-\widehat{\otimes}$-comodule algebra. We have the following identities in $A \widehat{\#}^{H} B$ :

$$
\begin{aligned}
(a \otimes 1)\left(a^{\prime} \otimes b^{\prime}\right) & =a a^{\prime} \otimes b^{\prime}, \\
(a \otimes b)\left(1 \otimes b^{\prime}\right) & =a \otimes b b^{\prime}, \\
(1 \otimes b)(a \otimes 1) & =\tau_{B, A}(b \otimes a), \\
(a \otimes b)\left(a^{\prime} \otimes b^{\prime}\right) & =(a \otimes 1) \tau_{B, A}\left(b \otimes a^{\prime}\right)\left(1 \otimes b^{\prime}\right) .
\end{aligned}
$$

Proof. Identities (5.3) and (5.5) are immediate from (5.2). To prove (5.4), let $\varepsilon^{\prime}: H \widehat{\otimes} \mathbb{C} \rightarrow \mathbb{C}$ be given by $\varepsilon^{\prime}(h \otimes \lambda)=\varepsilon_{\boldsymbol{H}}(h) \lambda$. We have a commutative diagram

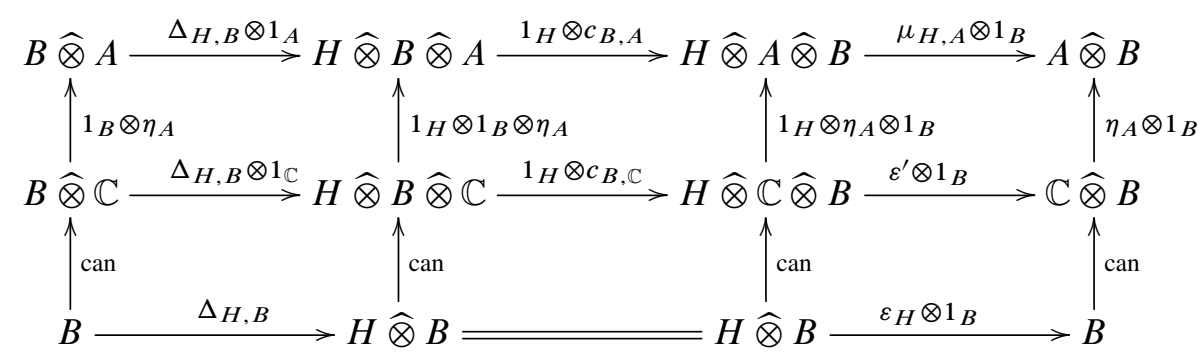

where the arrows marked by "can" are the canonical isomorphisms. Since $\left(\varepsilon_{H} \otimes\right.$ $\left.1_{B}\right) \Delta_{H, B}=1_{B}$, (5.4) now follows from (5.7). Identity (5.6) is immediate from (5.3)-(5.5).

Lemma 5.1 implies, in particular, that the maps

$$
\begin{aligned}
& i_{A}: A \rightarrow A \widehat{\#}^{H} B, \quad a \mapsto a \otimes 1, \\
& i_{B}: B \rightarrow A \widehat{\#}^{H} B, \quad b \mapsto 1 \otimes b,
\end{aligned}
$$

are $\widehat{\otimes}$-algebra homomorphisms. 
Definition 5.2. Given a $\widehat{\otimes}$-algebra $C$, we say that a pair $\left(\varphi_{A}: A \rightarrow C, \varphi_{B}: B \rightarrow C\right)$ of $\widehat{\otimes}$-algebra homomorphisms is compatible if the diagram

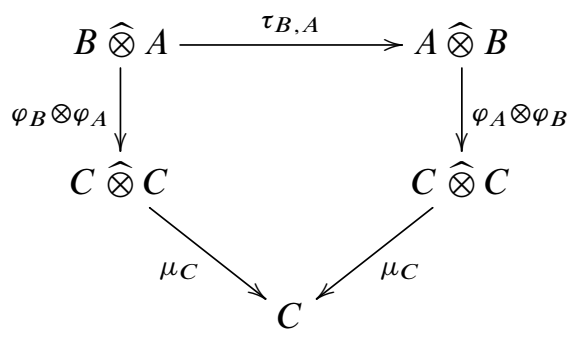

is commutative.

Proposition 5.3. Let $H$ be a $\widehat{\otimes}$-bialgebra, $A$ be a left $H-\widehat{\otimes}$-module algebra, and $B$ be a left $H$ - $\widehat{\otimes}$-comodule algebra. Let the homomorphisms $i_{A}, i_{B}$ be given by (5.8). Then

(i) the pair $\left(i_{A}, i_{B}\right)$ is compatible;

(ii) for each $\widehat{\otimes}$-algebra $C$ and each compatible pair $\left(\varphi_{A}: A \rightarrow C, \varphi_{B}: B \rightarrow C\right)$ of continuous homomorphisms there exists a unique continuous homomorphism $A \widehat{\#}^{H} B \rightarrow C$ making the diagram

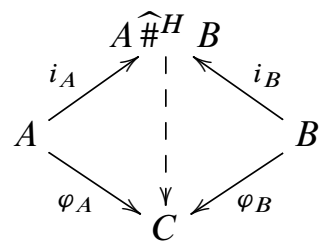

commute.

In other words, we have a natural isomorphism

$\operatorname{Hom}_{\widehat{\otimes} \text {-alg }}\left(A \widehat{\#}^{H} B, C\right) \cong\left\{\right.$ compatible $\left.\left(\varphi_{A}, \varphi_{B}\right) \in \operatorname{Hom}_{\widehat{\otimes} \text {-alg }}(A, C) \times \operatorname{Hom}_{\widehat{\otimes} \text {-alg }}(B, C)\right\}$,

where $\widehat{\otimes}$-alg is the category of all $\widehat{\otimes}$-algebras.

Proof.

(i) It is immediate from (5.3) and (5.5) that

$$
\mu_{A \widehat{\#}^{H} B}\left(i_{A} \otimes i_{B}\right)=1_{A \widehat{\otimes} B}, \quad \mu_{A \widehat{\#}^{H} B}\left(i_{B} \otimes i_{A}\right)=\tau_{B, A} .
$$

This readily implies that the pair $\left(i_{A}, i_{B}\right)$ is compatible.

(ii) Define $\psi: A \widehat{\#}^{H} B \rightarrow C$ by $\psi=\mu_{C}\left(\varphi_{A} \otimes \varphi_{B}\right)$. Clearly, $\psi$ makes (5.9) commute. We claim that $\psi$ is an algebra homomorphism. Indeed, by using (5.3), for each $a, a^{\prime} \in A$ and each $b^{\prime} \in B$ we obtain

$\psi\left((a \otimes 1)\left(a^{\prime} \otimes b^{\prime}\right)\right)=\psi\left(a a^{\prime} \otimes b^{\prime}\right)=\varphi_{A}(a) \varphi_{A}\left(a^{\prime}\right) \varphi_{B}\left(b^{\prime}\right)=\varphi_{A}(a) \psi\left(a^{\prime} \otimes b^{\prime}\right)$. 
By linearity and continuity, we conclude that

$$
\psi((a \otimes 1) u)=\varphi_{A}(a) \psi(u) \quad\left(a \in A, u \in A \widehat{\#}^{H} B\right) .
$$

Similarly, (5.4) yields

$$
\psi(u(1 \otimes b))=\psi(u) \varphi_{B}(b) \quad\left(b \in B, u \in A \widehat{\#}^{H} B\right) .
$$

Since $\left(\varphi_{A}, \varphi_{B}\right)$ is a compatible pair, we have

$$
\psi\left(\tau_{B, A}(b \otimes a)\right)=\varphi_{B}(b) \varphi_{A}(a) \quad(b \in B, a \in A) .
$$

Finally, by using (5.6) and (5.10)-(5.12), for each $a, a^{\prime} \in A$ and each $b, b^{\prime} \in B$ we obtain

$$
\begin{aligned}
\psi\left((a \otimes b)\left(a^{\prime} \otimes b^{\prime}\right)\right) & =\psi\left((a \otimes 1) \tau_{B, A}\left(b \otimes a^{\prime}\right)\left(1 \otimes b^{\prime}\right)\right) \\
& =\varphi_{A}(a) \varphi_{B}(b) \varphi_{A}\left(a^{\prime}\right) \varphi_{B}\left(b^{\prime}\right)=\psi(a \otimes b) \psi\left(a^{\prime} \otimes b^{\prime}\right) .
\end{aligned}
$$

Thus $\psi$ is an algebra homomorphism. The uniqueness of $\psi$ is immediate from the fact that $\operatorname{Im} i_{A}$ and $\operatorname{Im} i_{B}$ generate a dense subalgebra of $A \widehat{\#}^{H} B$.

Remark 5.4. Proposition 5.3 has an obvious "purely algebraic" version which characterizes the algebraic smash product $A \#^{H} B$.

Proposition 5.3 motivates the following definition.

Definition 5.5. Let $H$ be a $\widehat{\otimes}$-bialgebra, $A$ be a left $H$ - $\widehat{\otimes}$-module Arens-Michael algebra, and $B$ be a left $H$ - $\widehat{\otimes}$-comodule Arens-Michael algebra. The ArensMichael smash product of $A$ and $B$ over $H$ is an Arens-Michael algebra $A \#_{\mathrm{AM}}^{H} B$ together with a compatible pair $\left(i_{A}: A \rightarrow A \#_{\mathrm{AM}}^{H} B, i_{B}: B \rightarrow A \#_{\mathrm{AM}}^{H} B\right)$ of continuous homomorphisms such that for each Arens-Michael algebra $C$ and each compatible pair $\left(\varphi_{A}: A \rightarrow C, \varphi_{B}: B \rightarrow C\right)$ of continuous homomorphisms there exists a unique continuous homomorphism $A \#_{\text {AM }}^{H} B \rightarrow C$ making the diagram

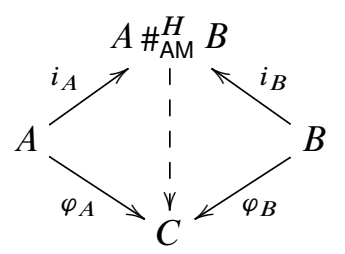

commute.

In other words, the Arens-Michael smash product is an Arens-Michael algebra $A \#_{\text {AM }}^{H} B$ together with a natural isomorphism

$\operatorname{Hom}_{\mathrm{AM}}\left(A \#_{\mathrm{AM}}^{H} B, C\right) \cong\left\{\right.$ compatible $\left.\left(\varphi_{A}, \varphi_{B}\right) \in \operatorname{Hom}_{\mathrm{AM}}(A, C) \times \operatorname{Hom}_{\mathrm{AM}}(B, C)\right\}$.

Proposition 5.3 easily implies that $A \#_{\mathrm{AM}}^{H} B$ exists. Indeed, we have

$$
A \#_{\mathrm{AM}}^{H} B=\left(A \widehat{\#}^{H} B\right) \text {. }
$$

Here is one more characterization of $A \#_{\mathrm{AM}}^{H} B$. 
Proposition 5.6. Let $H$ be a $\widehat{\otimes}$-bialgebra, A be a left $H$ - $\widehat{\otimes}$-module Arens-Michael algebra, and $\mathrm{B}$ be a left $\mathrm{H}-\widehat{\otimes}$-comodule Arens-Michael algebra. Denote by

$$
j_{A}: A \rightarrow A \widehat{*} B, \quad j_{B}: B \rightarrow A \widehat{*} B
$$

the canonical homomorphisms, and let $J$ be the closed two-sided ideal of $A \widehat{*} B$ generated by

$$
\operatorname{Im}\left(\mu_{A \widehat{*}_{B}}\left(j_{B} \otimes j_{A}-\left(j_{A} \otimes j_{B}\right) \tau_{B, A}\right)\right) .
$$

Finally, let $S$ be the completion of $(A \widehat{*} B) / J$, and let the homomorphisms $i_{A}: A \rightarrow S$ and $i_{B}: B \rightarrow S$ be the compositions of $j_{A}, j_{B}$ with the quotient map $A \widehat{*} B \rightarrow S$. Then $\left(S, i_{A}, i_{B}\right)$ is the Arens-Michael smash product of $A$ and $B$.

Proof. For each Arens-Michael algebra $C$ we have natural bijections

$$
\begin{aligned}
\operatorname{Hom}_{\mathrm{AM}}(S, C) & \cong\left\{\varphi \in \operatorname{Hom}_{\mathrm{AM}}(A \widehat{*} B, C): \varphi(J)=0\right\} \\
& \cong\left\{\varphi \in \operatorname{Hom}_{\mathrm{AM}}(A \widehat{*} B, C): \varphi \mu_{A \widehat{*} B}\left(j_{B} \otimes j_{A}-\left(j_{A} \otimes j_{B}\right) \tau_{B, A}\right)=0\right\} \\
& \cong\left\{\varphi \in \operatorname{Hom}_{\mathrm{AM}}(A \widehat{*} B, C): \mu_{C}(\varphi \otimes \varphi)\left(j_{B} \otimes j_{A}-\left(j_{A} \otimes j_{B}\right) \tau_{B, A}\right)=0\right\} \\
& \cong\left\{\varphi \in \operatorname{Hom}_{\mathrm{AM}}(A \widehat{*} B, C): \mu_{C}\left(\varphi j_{B} \otimes \varphi j_{A}-\left(\varphi j_{A} \otimes \varphi j_{B}\right) \tau_{B, A}\right)=0\right\} \\
& \cong\left\{\varphi \in \operatorname{Hom}_{\mathrm{AM}}(A \widehat{*} B, C):\left(\varphi j_{A}, \varphi j_{B}\right) \text { is compatible }\right\} \\
& \cong\left\{\operatorname{compatible}\left(\varphi_{A}, \varphi_{B}\right) \in \operatorname{Hom}_{\mathrm{AM}}(A, C) \times \operatorname{Hom}_{\mathrm{AM}}(B, C)\right\} \\
& \cong \operatorname{Hom}_{\mathrm{AM}}\left(A \#_{\mathrm{AM}}^{H} B, C\right) .
\end{aligned}
$$

Corollary 5.7. Let $H$ be a $\widehat{\otimes}$-bialgebra, A be a left $H$ - $\widehat{\otimes}$-module Arens-Michael algebra, and $B$ be a left $H$ - $\widehat{\otimes}$-comodule Arens-Michael algebra. If both $A$ and $B$ are holomorphically finitely generated, then so is $A \#_{\mathrm{AM}}^{H} B$.

Proof. Immediate from Proposition 5.6, Corollary 4.7, and Proposition 3.18.

So far we have two constructions of $A \#_{A M}^{H} B$, the one given by (5.14) and the one given by Proposition 5.6. However, both of them are rather implicit. In particular, they tell us almost nothing about the underlying locally convex space of $A \#_{\mathrm{AM}}^{H} B$. Fortunately, as we will see in Proposition 5.16 and Corollary 5.18, we actually have $A \#_{\mathrm{AM}}^{H} B=A \widehat{\#}^{H} H$ under appropriate conditions. To this end, we need some definitions and a lemma.

Definition 5.8. Let $X$ be a vector space, and let $\mathscr{T}$ be a set of linear operators on $X$. We say that a seminorm $\|\cdot\|$ on $X$ is $\mathscr{T}$-stable [37] if for each $T \in \mathscr{T}$ there exists $C>0$ such that for each $x \in X$ we have $\|T x\| \leq C\|x\|$. A subset $U \subseteq X$ is said to be $\mathscr{T}$-stable if for each $T \in \mathscr{T}$ there exists $C>0$ such that $T(U) \subseteq C U$.

The following is a "continuous version" of Definition 5.8. 
Definition 5.9. Let $A$ be a locally convex algebra, and let $X$ be a left $A$-module. We say that a seminorm $\|\cdot\|_{X}$ on $X$ is $A$-stable if there exists a continuous seminorm $\|\cdot\|_{A}$ on $A$ such that for all $a \in A, x \in X$ we have $\|a \cdot x\|_{X} \leq\|a\|_{A}\|x\|_{X}$. A subset $U \subseteq X$ is said to be $A$-stable if there exists a 0 -neighborhood $V \subseteq A$ such that $V \cdot U \subseteq U$.

Remark 5.10. Observe that a seminorm $\|\cdot\|$ is $\mathscr{T}$-stable (respectively, $A$-stable) if and only if so is the unit ball $\{x \in X:\|x\| \leq 1\}$.

Remark 5.11. Clearly, each $A$-stable seminorm on $X$ is stable with respect to the set $\{x \mapsto a \cdot x: a \in A\}$ of multiplication operators. If $A$ is endowed with the strongest locally convex topology, then the converse is also true. Indeed, suppose that a seminorm $\|\cdot\|_{X}$ on $X$ is stable with respect to $\{x \mapsto a \cdot x: a \in A\}$. Then $\|a\|_{A}=\sup \left\{\|a \cdot x\|_{X}:\|x\|_{X} \leq 1\right\}$ is a seminorm on $A$ satisfying $\|a \cdot x\|_{X} \leq$ $\|a\|_{A}\|x\|_{X}$. Thus $\|\cdot\|_{X}$ is $A$-stable.

Definition 5.12. Let $B$ be an Arens-Michael algebra, and let $\mathscr{T}$ be a set of continuous linear operators on $B$. We say that $\mathscr{T}$ is $m$-localizable [38] if there exists a defining family of submultiplicative, $\mathscr{T}$-stable seminorms on $B$. A linear operator $T: B \rightarrow B$ is $m$-localizable if so is the singleton $\{T\}$.

Finally, we have the following "continuous" version of Definition 5.12.

Definition 5.13. Let $A$ be a locally convex algebra, and let $B$ be an Arens-Michael algebra endowed with a left $A$-module structure. We say that the action of $A$ on $B$ is $m$-localizable if there exists a defining family of submultiplicative, $A$-stable seminorms on $B$. Equivalently, the action of $A$ on $B$ is $m$-localizable if there exists a base of idempotent, $A$-stable 0 -neighborhoods in $B$.

Remark 5.14. Let $A$ be an Arens-Michael algebra, and let $B$ be an Arens-Michael algebra endowed with a left $A$ - $\widehat{\otimes}$-module structure. By [38, Proposition 3.4], there always exists a defining family of $A$-stable seminorms on $B$. However, it is unclear whether it is possible to find a defining family of seminorms that are $A$-stable and submultiplicative simultaneously.

The following lemma (see [37, Corollary 1.3]) is a special case of a useful result due to Mitiagin, Rolewicz, and Żelazko [32].

Lemma 5.15. Let $A$ be a topological algebra. Suppose that $A$ has a base $\mathscr{U}$ of absolutely convex 0-neighborhoods with the property that for each $V \in \mathscr{U}$ there exist $U \in \mathscr{U}$ and $C>0$ such that $U V \subset C V$. Then $A$ is locally $m$-convex.

Recall a notation from the theory of topological vector spaces (see, e.g., [16, 24, 42]). Let $E$ and $F$ be complete locally convex spaces, and let $U \subseteq E$ and $V \subseteq F$ be 0-neighborhoods. By a standard abuse of notation, we let $\overline{\Gamma(U \otimes V)}$ denote the closed absolutely convex hull of the set $U \otimes V=\{u \otimes v: u \in U, v \in V\} \subset E \widehat{\otimes} F$. If now $\mathscr{U}$ (respectively, $\mathscr{V}$ ) is a base of 0-neighborhoods in $E$ (respectively, $F$ ), then $\{\overline{\Gamma(U \otimes V)}: U \in \mathscr{U}, V \in \mathscr{V}\}$ is a base of 0-neighborhoods in $E \widehat{\otimes} F$ (loc. cit.). 
Now we are in a position to show that, under appropriate conditions, the $\widehat{\otimes}$-smash product and the Arens-Michael smash product are the same.

Proposition 5.16. Let $H$ be $a \widehat{\otimes}$-bialgebra, A be a left $H$ - $\widehat{\otimes}$-module Arens-Michael algebra, and $B$ be a left $H$ - $\widehat{\otimes}$-comodule Arens-Michael algebra. Suppose that the action of $H$ on $A$ is $m$-localizable. Then $A \widehat{\#}^{H} B$ is an Arens-Michael algebra. Equivalently, $A \#_{\mathrm{AM}}^{H} B=A \widehat{\#}^{H} B$.

Proof. Let $\mathscr{U}$ be a base of idempotent, $H$-stable 0-neighborhoods in $A$, and let $\mathscr{V}$ be a base of idempotent 0 -neighborhoods in $B$. Then the family

$$
\mathscr{B}=\{\overline{\Gamma(U \otimes V)}: U \in \mathscr{U}, V \in \mathscr{V}\}
$$

is a 0-neighborhood base in $A \widehat{\#}^{H} B$. Given $U \in \mathscr{U}$ and $V \in \mathscr{V}$, let $W \subseteq H$ be a 0-neighborhood such that $W \cdot U \subseteq U$. Choose $V^{\prime} \in \mathscr{V}$ such that $\Delta_{H, B}\left(V^{\prime}\right) \subseteq$ $\overline{\Gamma(W \otimes V)}$. We claim that

$$
\overline{\Gamma\left(U \otimes V^{\prime}\right)} \overline{\Gamma(U \otimes V)} \subseteq \overline{\Gamma(U \otimes V)} .
$$

Indeed, take $u_{1}, u_{2} \in U, v_{1} \in V^{\prime}$, and $v_{2} \in V$. Then $\Delta_{H, B}\left(v_{1}\right) \in \overline{\Gamma(W \otimes V)}$. Since $W \cdot U \subseteq U$, i.e., $\mu_{H, A}(W \otimes U) \subseteq U$, it follows that

$$
\begin{aligned}
\tau_{B, A}\left(v_{1} \otimes u_{2}\right) & =\left(\mu_{H, A} \otimes 1_{B}\right)\left(1_{H} \otimes c_{B, A}\right)\left(\Delta_{H, B}\left(v_{1}\right) \otimes u_{2}\right) \\
& \in\left(\mu_{H, A} \otimes 1_{B}\right)\left(1_{H} \otimes c_{B, A}\right)(\overline{\Gamma(W \otimes V \otimes U)}) \\
& =\left(\mu_{H, A} \otimes 1_{B}\right)(\overline{\Gamma(W \otimes U \otimes V)} \subseteq \overline{\Gamma(U \otimes V)} .
\end{aligned}
$$

Therefore,

$$
\begin{aligned}
\left(u_{1} \otimes v_{1}\right)\left(u_{2} \otimes v_{2}\right) & =\left(\mu_{A} \otimes \mu_{B}\right)\left(u_{1} \otimes \tau_{B, A}\left(v_{1} \otimes u_{2}\right) \otimes v_{2}\right) \\
& \in\left(\mu_{A} \otimes \mu_{B}\right)(U \otimes \overline{\Gamma(U \otimes V)} \otimes V) \\
& \subseteq\left(\mu_{A} \otimes \mu_{B}\right)(\overline{\Gamma(U \otimes U \otimes V \otimes V)}) \subseteq \overline{\Gamma(U \otimes V)} .
\end{aligned}
$$

This proves (5.15). Thus the base $\mathscr{B}$ satisfies the conditions of Lemma 5.15, whence $A \widehat{\#}^{H} B$ is an Arens-Michael algebra.

Proposition 5.17. Let $H$ be a Banach bialgebra, and let $A$ be a left $H$ - $\widehat{\otimes}$-module Arens-Michael algebra. Then the action of $H$ on $A$ is m-localizable.

Proof. Let $\left\{\|\cdot\|_{\lambda}: \lambda \in \Lambda\right\}$ be a directed defining family of submultiplicative seminorms on $A$. Without loss of generality, we assume that the norm $\|\cdot\|$ on $H$ is submultiplicative, and that $\left\|1_{H}\right\|=1$. Given $\lambda \in \Lambda$, find $\mu \in \Lambda$ and $C_{1}>0$ such that

$$
\|h \cdot a\|_{\lambda} \leq C_{1}\|h\|\|a\|_{\mu} \quad(h \in H, a \in A) .
$$

Define a seminorm $\|\cdot\|_{\lambda}^{\prime}$ on $A$ by

$$
\|a\|_{\lambda}^{\prime}=\sup \left\{\|h \cdot a\|_{\lambda}:\|h\| \leq 1\right\} .
$$


By (5.16), we have $\|\cdot\|_{\lambda}^{\prime} \leq C_{1}\|\cdot\|_{\mu}$, whence $\|\cdot\|_{\lambda}^{\prime}$ is a continuous seminorm on $A$. Letting $h=1$, we see that $\|\cdot\|_{\lambda} \leq\|\cdot\|_{\lambda}^{\prime}$. Let now $h \in H, a \in A$, and assume that $\|h\| \leq 1$. Then

$$
\|h \cdot a\|_{\lambda}^{\prime}=\sup \left\{\left\|h_{1} h \cdot a\right\|_{\lambda}:\left\|h_{1}\right\| \leq 1\right\} \leq\|a\|_{\lambda}^{\prime},
$$

due to the submultiplicativity of $\|\cdot\|$. Hence

$$
\|h \cdot a\|_{\lambda}^{\prime} \leq\|h\|\|a\|_{\lambda}^{\prime} \quad(h \in H, a \in A),
$$

and so $\|\cdot\|_{\lambda}^{\prime}$ is $H$-stable.

Let $\|\cdot\|_{\pi}$ denote the projective tensor norm on $H \widehat{\otimes} H$, and let $\|\cdot\|_{\lambda, \pi}^{\prime}$ denote the projective tensor seminorm on $A \widehat{\otimes} A$ associated to $\|\cdot\|_{\lambda}^{\prime}$. Observe that (5.17) implies the estimate

$$
\|u \cdot v\|_{\lambda, \pi}^{\prime} \leq\|u\|_{\pi}\|v\|_{\lambda, \pi}^{\prime} \quad(u \in H \widehat{\otimes} H, v \in A \widehat{\otimes} A) .
$$

Choose $C \geq 1$ such that

$$
\left\|\Delta_{H}(h)\right\|_{\pi} \leq C\|h\| \quad(h \in H) .
$$

For each $a, b \in A$ we have

$$
\begin{aligned}
\|a b\|_{\lambda}^{\prime} & =\sup \left\{\|h \cdot a b\|_{\lambda}:\|h\| \leq 1\right\} & & \\
& =\sup \left\{\left\|\mu_{A}\left(\Delta_{H}(h) \cdot(a \otimes b)\right)\right\|_{\lambda}:\|h\| \leq 1\right\} & & \left(\text { since } \mu_{A} \text { is an } H\right. \text {-module morphism) } \\
& \leq \sup \left\{\left\|\Delta_{H}(h) \cdot(a \otimes b)\right\|_{\lambda, \pi}:\|h\| \leq 1\right\} & & \left(\text { since }\|\cdot\|_{\lambda}\right. \text { is submultiplicative) } \\
& \leq \sup \left\{\left\|\Delta_{H}(h) \cdot(a \otimes b)\right\|_{\lambda, \pi}^{\prime}:\|h\| \leq 1\right\} & & \left(\text { since }\|\cdot\|_{\lambda} \leq\|\cdot\|_{\lambda}^{\prime}\right) \\
& \leq \sup \left\{\left\|\Delta_{H}(h)\right\|_{\pi}\|a \otimes b\|_{\lambda, \pi}^{\prime}:\|h\| \leq 1\right\} & & (\text { by (5.18)) } \\
& \leq C\|a \otimes b\|_{\lambda, \pi}^{\prime} & & \\
& =C\|a\|_{\lambda}^{\prime}\|b\|_{\lambda}^{\prime} . & &
\end{aligned}
$$

Hence $\|\cdot\|_{\lambda}^{\prime \prime}=C\|\cdot\|_{\lambda}^{\prime}$ is a continuous, $H$-stable, submultiplicative seminorm on $A$, and $\|\cdot\|_{\lambda} \leq\|\cdot\|_{\lambda}^{\prime \prime}$. Therefore $\left\{\|\cdot\|_{\lambda}^{\prime \prime}: \lambda \in \Lambda\right\}$ is a directed defining family of $H$-stable, submultiplicative seminorms on $A$, and the action of $H$ on $A$ is $m$-localizable.

Corollary 5.18. Let $H$ be a Banach bialgebra, $A$ be a left $H-\widehat{\otimes}$-module ArensMichael algebra, and $B$ be a left $H$ - $\widehat{\otimes}$-comodule Arens-Michael algebra. Then $A \widehat{\#}^{H} B$ is an Arens-Michael algebra. Equivalently, $A \#_{\mathrm{AM}}^{H} B=A \widehat{\#}^{H} B$.

Proof. Combine Propositions 5.16 and 5.17.

Remark 5.19. An inspection of the above proof shows that Proposition 5.17 holds for each Arens-Michael bialgebra $H$ that can be represented as an inverse limit of Banach bialgebras. Equivalently, this means that the topology on $H$ can be generated 
by a family $\left\{\|\cdot\|_{\lambda}: \lambda \in \Lambda\right\}$ of submultiplicative seminorms with the additional property that $\left\|\Delta_{H}(h)\right\|_{\lambda, \pi} \leq C_{\lambda}\|h\|_{\lambda}(h \in H)$, for suitable constants $C_{\lambda}>0$. We restrict ourselves to Banach bialgebras mostly because this is enough for our purposes, and also because the vast majority of natural nonnormable Arens-Michael bialgebras do not have the above property. We do not know, however, whether Proposition 5.17 holds for each Arens-Michael bialgebra $H$.

We now specialize to smash products coming from semigroup actions and semigroup graded algebras. Given a unital semigroup $S$, we endow the Banach algebra $\ell^{1}(S)$ with the structure of a Banach bialgebra by letting

$$
\Delta(s)=s \otimes s, \quad \varepsilon(s)=1 \quad(s \in S) .
$$

It is elementary to check that $\Delta$ and $\varepsilon$ uniquely extend to continuous comultiplication and counit on $\ell^{1}(S)$, respectively, and that $\left(\ell^{1}(S), \Delta, \varepsilon\right)$ is indeed a Banach bialgebra.

Proposition 5.20. Let $S$ be a unital semigroup, and let $A$ be a $\widehat{\otimes}$-algebra.

(i) Suppose that $S$ acts on $A$ by endomorphisms in such a way that the action is equicontinuous. Then $A$ is a left $\ell^{1}(S)-\widehat{\otimes}$-module algebra via the map

$$
\mu_{S, A}: \ell^{1}(S) \times A \rightarrow A, \quad\left(\sum_{s \in S} c_{s} s, a\right) \mapsto \sum_{s \in S} c_{s}(s \cdot a) .
$$

(ii) Conversely, if $A$ is endowed with a left $\ell^{1}(S)-\widehat{\otimes}$-module algebra structure, then the respective action of $S$ on $A$ is equicontinuous.

Proof. (i) Let $\left\{\|\cdot\|_{\lambda}: \lambda \in \Lambda\right\}$ be a directed defining family of seminorms on $A$. Given $\lambda \in \Lambda$, find $\mu \in \Lambda$ and $C>0$ such that $\|s \cdot a\|_{\lambda} \leq C\|a\|_{\mu}$ for all $s \in S, a \in A$. Then for each $h=\sum_{s} c_{s} s \in \ell^{1}(S)$ we have

$$
\sum_{s \in S}\left\|c_{s}(s \cdot a)\right\|_{\lambda} \leq C \sum_{s \in S}\left|c_{s}\right|\|a\|_{\mu}=C\|h\|\|a\|_{\mu} .
$$

Thus the family $\left(c_{S}(s \cdot a)\right)_{s \in S}$ is absolutely summable in $A$, and (5.20) yields a jointly continuous bilinear map from $\ell^{1}(S) \times A$ to $A$. The axioms of an $\ell^{1}(S)$ - $\widehat{\otimes}$-module algebra are readily verified.

(ii) Let $\left\{\|\cdot\|_{\lambda}: \lambda \in \Lambda\right\}$ be a directed defining family of seminorms on $A$. Given $\lambda \in \Lambda$, choose $\mu \in \Lambda$ and $C>0$ such that

$$
\|h \cdot a\|_{\lambda} \leq C\|h\|\|a\|_{\mu} \quad\left(h \in \ell^{1}(S), a \in A\right) .
$$

Letting $h=s \in S$, we see that the action of $S$ on $A$ is equicontinuous.

To introduce an appropriate "analytic" version of a semigroup graded algebra, we need a definition from the theory of locally convex spaces. 
Definition 5.21. Let $E$ be a complete locally convex space. Following [6, 3.3], we say that a family $\left\{E_{s}: s \in S\right\}$ of vector subspaces of $E$ is an absolute Schauder decomposition of $E$ if the following conditions hold:

(i) for each $x \in E$ there exists a unique family $\left(x_{s}\right)_{s \in S} \in \prod_{s \in S} E_{s}$ such that $x=\sum_{s \in S} x_{s}$

(ii) for each continuous seminorm $\|\cdot\|$ on $E$ we have $\|x\|^{\prime}=\sum_{s \in S}\left\|x_{s}\right\|<\infty$ (i.e., the family $\left(x_{s}\right)_{s \in S}$ is absolutely summable to $x$ ), and, moreover, the seminorm $\|\cdot\|^{\prime}$ is continuous.

We say that a seminorm $\|\cdot\|$ on $E$ is graded if for each $x \in E$ we have $\|x\|=$ $\sum_{s \in S}\left\|x_{s}\right\|$. It follows from the above definition that the family of all continuous graded seminorms generates the original topology on $E$.

Definition 5.22. Let $S$ be a unital semigroup, and let $A$ be a $\widehat{\otimes}$-algebra. By an absolute $S$-grading on $A$ we mean an absolute Schauder decomposition $\left\{A_{s}: s \in S\right\}$ of $A$ such that for each $s, t \in S$ we have $A_{s} A_{t} \subseteq A_{s t}$. An absolutely $S$-graded $\widehat{\otimes}$ algebra is a $\widehat{\otimes}$-algebra endowed with an absolute $S$-grading.

Similarly to the purely algebraic case, it is easy to see that for each $a, b \in A$ and each $r \in S$ we have $(a b)_{r}=\sum_{s t=r} a_{s} b_{t}$. We also have $1 \in A_{e}$, where $e$ is the identity element of $S$ (cf. [33, 1.1.1]).

Proposition 5.23. Let $S$ be a unital semigroup, and let $A$ be a $\widehat{\otimes}$-algebra.

(i) If $A$ is endowed with an absolute $S$-grading, then $A$ is a left $\ell^{1}(S)$ - $\widehat{\otimes}$-comodule algebra via the map

$$
\Delta_{S, A}: A \rightarrow \ell^{1}(S) \widehat{\otimes} A, \quad a \mapsto \sum_{s \in S} s \otimes a_{s} \quad(a \in A) .
$$

(ii) Conversely, if $A$ is endowed with a left $\ell^{1}(S)-\widehat{\otimes}$-comodule algebra structure, then the subspaces $A_{s}=\left\{a \in A: \Delta_{S, A}(a)=s \otimes a\right\}$ form an absolute $S$-grading on $A$.

Proof. Let $\left\{\|\cdot\|_{\lambda}: \lambda \in \Lambda\right\}$ be a directed defining family of seminorms on $A$. For each $\lambda \in \Lambda$, we will use the same notation $\|\cdot\|_{\lambda}$ for the respective projective tensor seminorm on $\ell^{1}(S) \widehat{\otimes} A$.

(i) Suppose that $A$ is absolutely $S$-graded. Without loss of generality, we may assume that each seminorm $\|\cdot\|_{\lambda}$ is graded. For each $a \in A$, we have

$$
\sum_{s \in S}\left\|s \otimes a_{S}\right\|_{\lambda}=\sum_{s \in S}\left\|a_{s}\right\|_{\lambda}=\|a\|_{\lambda}
$$

Hence the family $\left(s \otimes a_{s}\right)_{s \in S}$ is absolutely summable in $\ell^{1}(S) \widehat{\otimes} A$, and (5.23) yields a continuous linear map from $A$ to $\ell^{1}(S) \widehat{\otimes} A$. The axioms of a left $\ell^{1}(S)-\widehat{\otimes}$ comodule algebra are readily verified. 
(ii) Conversely, assume that $A$ is a left $\ell^{1}(S)-\widehat{\otimes}$-comodule algebra. Recall [35, 7.2.3] that there exists a topological isomorphism between $\ell^{1}(S) \widehat{\otimes} A$ and the locally convex space $\ell^{1}(S, A)$ of all absolutely summable families $\left(a_{s}\right)_{s \in S}$ in $A$. Explicitly, the isomorphism takes each $\left(a_{s}\right)_{s \in S} \in \ell^{1}(S, A)$ to $\sum_{s \in S} s \otimes a_{s} \in \ell^{1}(S) \widehat{\otimes} A$, and for all $\lambda \in \Lambda$ we have $\left\|\sum_{s \in S} s \otimes a_{s}\right\|_{\lambda}=\sum_{s \in S}\left\|a_{s}\right\|_{\lambda}$. Hence for each $a \in A$ there exists a unique absolutely summable family $\left(a_{s}\right)_{s \in S}$ in $A$ such that the coaction $\Delta_{S, A}$ of $\ell^{1}(S)$ on $A$ is given by (5.23). By coassociativity, we have

$$
\sum_{s \in S} s \otimes \Delta_{S, A}\left(a_{s}\right)=\sum_{s \in S} s \otimes s \otimes a_{s},
$$

whence $\Delta_{S, A}\left(a_{s}\right)=s \otimes a_{s}$, i.e., $a_{s} \in A_{s}$. We also have

$$
a=\left(\varepsilon \otimes 1_{A}\right)\left(\Delta_{S, A}(a)\right)=\sum_{s \in S} a_{S} \quad(a \in A) .
$$

If $\left(a_{s}^{\prime}\right)_{s \in S} \in \prod_{s} A_{s}$ is another summable family such that $a=\sum_{s \in S} a_{s}^{\prime}$, then applying $\Delta_{S, A}$ yields $\sum_{s \in S} S \otimes a_{s}=\sum_{s \in S} s \otimes a_{s}^{\prime}$, whence $a_{s}^{\prime}=a_{s}$ for all $s \in S$. Thus condition (i) of Definition 5.21 is satisfied. Clearly, for each $\lambda \in \Lambda$ the seminorm $\|a\|_{\lambda}^{\prime}=\left\|\Delta_{S, A}(a)\right\|_{\lambda}$ is continuous on $A$. On the other hand, $\|a\|_{\lambda}^{\prime}=\sum_{s \in S}\left\|a_{s}\right\|_{\lambda}$. Thus condition (ii) of Definition 5.21 is also satisfied, and so $\left\{A_{s}: s \in S\right\}$ is an absolute Schauder decomposition of $A$. Finally, for each $a \in A_{s}$ and $b \in A_{t}$ we have $\Delta_{S, A}(a b)=(s \otimes a)(t \otimes b)=s t \otimes a b$, i.e., $a b \in A_{s t}$. This completes the proof.

Theorem 5.24. Let $A$ be a $\widehat{\otimes}$-algebra. Suppose that a unital semigroup $S$ acts on $A$ by endomorphisms in such a way that the action is equicontinuous. Let also $B$ be an absolutely $S$-graded $\widehat{\otimes}$-algebra. Then there exists a unique $\widehat{\otimes}$-algebra structure on $A \widehat{\otimes} B$ such that

$$
\begin{aligned}
\left(a_{1} \otimes 1\right)\left(a_{2} \otimes 1\right) & =a_{1} a_{2} \otimes 1 & & \left(a_{1}, a_{2} \in A\right) ; \\
\left(1 \otimes b_{1}\right)\left(1 \otimes b_{2}\right) & =1 \otimes b_{1} b_{2} & & \left(b_{1}, b_{2} \in B\right) ; \\
(a \otimes 1)(1 \otimes b) & =a \otimes b & & (a \in A, b \in B) ; \\
\left(1 \otimes b_{s}\right)(a \otimes 1) & =(s \cdot a) \otimes b_{s} & & \left(a \in A, b_{s} \in B_{s}, s \in S\right) .
\end{aligned}
$$

The resulting $\widehat{\otimes}$-algebra $A \widehat{\#}^{S} B$ is equal to $A \widehat{\#}^{1}(S) B$. If, in addition, $A$ and $B$ are Arens-Michael algebras, then so is $A \widehat{\#}^{S} B$. Finally, if both $A$ and $B$ are holomorphically finitely generated, then so is $A \widehat{\#}^{S} B$.

Proof. Applying Propositions 5.20 and 5.23, we obtain a left $\ell^{1}(S)$ - $\widehat{\otimes}$-module algebra structure on $A$ and a left $\ell^{1}(S)-\widehat{\otimes}$-comodule algebra structure on $B$. Let $A \widehat{\#}^{S} B=A \widehat{\#}^{1}(S) B$. Relations (5.24)-(5.27) are immediate from Lemma 5.1 modulo the fact that $\Delta_{S, B}\left(b_{s}\right)=s \otimes b$. By (5.26), the elements $a \otimes 1(a \in A)$ 
and $1 \otimes b_{s}\left(s \in S, b_{s} \in B_{S}\right)$ generate a dense subalgebra of $A \widehat{\#}^{S} B$, whence the multiplication on $A \widehat{\#}^{S} B$ is uniquely determined by (5.24)-(5.27). If both $A$ and $B$ are Arens-Michael algebras, then Corollary 5.18 implies that $A \widehat{\#}^{S} B$ is an ArensMichael algebra too. The last assertion follows from Corollary 5.7.

\section{Skew holomorphic functions}

A construction closely related to smash products is that of an Ore extension, or a skew polynomial ring (see, e.g., [21,30]). Similarly to free products and smash products, Ore extensions also have natural "analytic" counterparts [38].

Let $A$ be an algebra, and let $\sigma$ be an endomorphism of $A$. Recall that a linear map $\delta: A \rightarrow A$ is a $\sigma$-derivation if $\delta(a b)=\delta(a) b+\sigma(a) \delta(b)$ for all $a, b \in A$. Suppose now that $A$ is an Arens-Michael algebra, $\sigma$ is an endomorphism of $A$, and $\delta$ is a $\sigma$-derivation such that $\{\sigma, \delta\}$ is $m$-localizable. By [38, Proposition 4.3], there exists a unique continuous multiplication on $\mathscr{O}(\mathbb{C}, A)$ such that the canonical embeddings

$$
\begin{aligned}
\mathscr{O}(\mathbb{C}) & \hookrightarrow \mathscr{O}(\mathbb{C}, A), \quad f \mapsto f \otimes 1, \\
A & \hookrightarrow \mathscr{O}(\mathbb{C}, A), \quad a \mapsto 1 \otimes a,
\end{aligned}
$$

are algebra homomorphisms, and such that

$$
z a=\sigma(a) z+\delta(a) \quad(a \in A),
$$

where $z \in \mathscr{O}(\mathbb{C}) \subset \mathscr{O}(\mathbb{C}, A)$ is the complex coordinate. The resulting $\widehat{\otimes}$-algebra is denoted by $\mathscr{O}(\mathbb{C}, A ; \sigma, \delta)$ and is called the Arens-Michael Ore extension (or the analytic Ore extension) of $A$ via $\{\sigma, \delta\}$. By [38, Proposition 4.5], $\mathscr{O}(\mathbb{C}, A ; \sigma, \delta)$ is an Arens-Michael algebra.

By [38, Proposition 4.4 and Remark 4.6], $\mathscr{O}(\mathbb{C}, A ; \sigma, \delta)$ has the universal property that for each Arens-Michael algebra $B$, each continuous homomorphism $\varphi: A \rightarrow B$, and each $x \in B$ satisfying $x \varphi(a)=\varphi(\sigma(a)) x+\varphi(\delta(a))(a \in A)$, there exists a unique continuous homomorphism $\psi: \mathscr{O}(\mathbb{C}, A ; \sigma, \delta) \rightarrow B$ such that $\left.\psi\right|_{A}=\varphi$ and $\psi(z)=x$. In other words, there is a natural bijection

$$
\begin{aligned}
\operatorname{Hom}_{\mathrm{AM}}(\mathscr{O}(\mathbb{C}, A ; \sigma, \delta), B) \cong\left\{\begin{array}{l}
(\varphi, x) \in \operatorname{Hom}_{\mathrm{AM}}(A, B) \times B: \\
x \varphi(a)=\varphi(\sigma(a)) x+\varphi(\delta(a)) \forall a \in A\} .
\end{array}\right.
\end{aligned}
$$

Remark 6.1. The algebra $\mathscr{O}(\mathbb{C}, A ; \sigma, \delta)$ can be viewed as an analytic analog of the algebraic Ore extension $A[z ; \sigma, \delta]$ (see, e.g., [21, 1.7]). Note that $A[z ; \sigma, \delta]$ is a dense subalgebra of $\mathscr{O}(\mathbb{C}, A ; \sigma, \delta)$. In fact, it is rather easy to deduce from (6.3) that, if we endow $A[z ; \sigma, \delta]$ with a suitable topology, then $\mathscr{O}(\mathbb{C}, A ; \sigma, \delta)$ becomes the ArensMichael envelope of $A[z ; \sigma, \delta]$. 
Proposition 6.2. Let $A$ be an Arens-Michael algebra, $\sigma$ be an endomorphism of $A$, and $\delta$ be a $\sigma$-derivation on $A$ such that $\{\sigma, \delta\}$ is m-localizable. Identify $\mathscr{O}(\mathbb{C})$ and $A$ with the respective subalgebras of $\mathscr{O}(\mathbb{C}) \widehat{*} A$ via the canonical embeddings $j_{\mathscr{O}(\mathbb{C})}$ and $j_{A}$, and let $J$ be the closed two-sided ideal of $\mathscr{O}(\mathbb{C}) \widehat{*} A$ generated by

$$
\{z a-\sigma(a) z-\delta(a): a \in A\} .
$$

Finally, let $S$ be the completion of $(\mathscr{O}(\mathbb{C}) \widehat{*} A) / J$. Then there exists a unique topological algebra isomorphism $S \cong \mathscr{O}(\mathbb{C}, A ; \sigma, \delta)$ such that $z+J \mapsto z$ and $a+J \mapsto$ a for all $a \in A$.

We omit the proof since it is similar to that of Proposition 5.6 modulo (6.3) and the natural bijection $\operatorname{Hom}_{\mathrm{AM}}(\mathscr{O}(\mathbb{C}), B) \cong B(B \in \mathrm{AM})$.

Corollary 6.3. Let $A$ be an Arens-Michael algebra, $\sigma$ be an endomorphism of $A$, and $\delta$ be a $\sigma$-derivation on $A$ such that $\{\sigma, \delta\}$ is $m$-localizable. If $A$ is holomorphically finitely generated, then so is $\mathscr{O}(\mathbb{C}, A ; \sigma, \delta)$.

If $\sigma=1_{A}$ (respectively, if $\delta=0$ ), then the algebra $\mathscr{O}(\mathbb{C}, A ; \sigma, \delta)$ is denoted by $\mathscr{O}(\mathbb{C}, A ; \delta)$ (respectively, $\mathscr{O}(\mathbb{C}, A ; \sigma)$ ). These algebras can be interpreted as ArensMichael smash products as follows (for details, see [38, Remarks 4.2-4.4]). Observe that the Fréchet-Arens-Michael algebra $\mathscr{O}(\mathbb{C})$ can be made into a $\widehat{\otimes}$-bialgebra in two different ways. The first way is to use the additive structure on $\mathbb{C}$ and to define a comultiplication $\Delta_{\text {add }}$ and a counit $\varepsilon_{\text {add }}$ on $\mathscr{O}(\mathbb{C})$ by

$$
\Delta_{\text {add }}(f)(z, w)=f(z+w), \quad \varepsilon_{\text {add }}(f)=f(0) \quad(f \in \mathscr{O}(\mathbb{C})) .
$$

The resulting Arens-Michael bialgebra (which is in fact an Arens-Michael Hopf algebra) will be denoted by $\mathscr{O}\left(\mathbb{C}_{\text {add }}\right)$. Alternatively, we can use the multiplicative structure on $\mathbb{C}$ to define a comultiplication $\Delta_{\text {mult }}$ and a counit $\varepsilon_{\text {mult }}$ on $\mathscr{O}(\mathbb{C})$ by

$$
\Delta_{\text {mult }}(f)(z, w)=f(z w), \quad \varepsilon_{\text {mult }}(f)=f(1) \quad(f \in \mathscr{O}(\mathbb{C})) .
$$

The resulting Arens-Michael bialgebra will be denoted by $\mathscr{O}\left(\mathbb{C}_{\text {mult }}\right)$. If now $A$ is an Arens-Michael algebra and $\delta$ is an $m$-localizable derivation on $A$, then $A$ can be made into an $\mathscr{O}\left(\mathbb{C}_{\text {add }}\right)$ - $\widehat{\otimes}$-module algebra in such a way that $z \cdot a=\delta(a)(a \in A)$. Moreover, the action of $\mathscr{O}\left(\mathbb{C}_{\text {add }}\right)$ on $A$ is easily seen to be $m$-localizable, and we have topological algebra isomorphisms

$$
\mathscr{O}(\mathbb{C}, A ; \delta) \cong A \widehat{\#} \mathscr{O}\left(\mathbb{C}_{\text {add }}\right) \cong A \#_{\text {AM }} \mathscr{O}\left(\mathbb{C}_{\text {add }}\right) .
$$

Similarly, if $\sigma$ is an $m$-localizable endomorphism of $A$, then $A$ can be made into an $\mathscr{O}\left(\mathbb{C}_{\text {mult }}\right)-\widehat{\otimes}$-module algebra in such a way that $z \cdot a=\sigma(a)(a \in A)$. Moreover, the action of $\mathscr{O}\left(\mathbb{C}_{\text {mult }}\right)$ on $A$ is easily seen to be $m$-localizable, and we have topological algebra isomorphisms

$$
\mathscr{O}(\mathbb{C}, A ; \sigma) \cong A \widehat{\#} \mathscr{O}\left(\mathbb{C}_{\text {mult }}\right) \cong A \#_{\mathrm{AM}} \mathscr{O}\left(\mathbb{C}_{\text {mult }}\right) .
$$


Of course, the isomorphisms (6.4) and (6.5) come from their algebraic versions $A[z ; \delta] \cong A \# \mathscr{O}^{\mathrm{reg}}\left(\mathbb{C}_{\mathrm{add}}\right) \cong A \# U(\mathbb{C})$ (where $U(\mathbb{C})$ is the enveloping algebra of the abelian Lie algebra $\mathbb{C}$ ) and $A[z ; \sigma] \cong A \# \mathscr{O}^{\text {reg }}\left(\mathbb{C}_{\text {mult }}\right) \cong A \# \mathbb{C} \mathbb{Z}_{+}$(where $\mathbb{C Z}_{+}$ is the semigroup algebra of the additive semigroup $\left.\mathbb{Z}_{+}\right)$.

Our next goal is to extend the definition of $\mathscr{O}(\mathbb{C}, A ; \sigma)$ to the case of $A$-valued functions on a domain $D \subseteq \mathbb{C}^{n}$ satisfying some additional conditions. In what follows, we let $z_{1}, \ldots, z_{n}$ denote the coordinates on $\mathbb{C}^{n}$. Recall that a subset $D \subseteq \mathbb{C}^{n}$ is balanced if for each $\lambda \in \mathbb{C}$ with $|\lambda| \leq 1$ we have $\lambda D \subseteq D$. Given a balanced domain $D \subseteq \mathbb{C}^{n}$ and $f \in \mathscr{O}(D)$, let $f_{m}$ denote the homogeneous polynomial of total degree $m \in \mathbb{Z}_{+}$that appears in the Taylor expansion of $f$ at 0 . Explicitly,

$$
f_{m}(z)=\sum_{\substack{k \in \mathbb{Z}_{+}^{n} \\|k|=m}} \frac{D^{k} f(0)}{k !} z^{k} .
$$

By [6, Proposition 3.36], the series $\sum_{m \in \mathbb{Z}_{+}} f_{m}$ absolutely converges in $\mathscr{O}(D)$, and we have $f=\sum_{m \in \mathbb{Z}_{+}} f_{m}$. Moreover (loc. cit.), for each compact set $K \subset D$ the seminorm

$$
\|f\|_{K}^{\prime}=\sum_{m \in \mathbb{Z}_{+}}\left\|f_{m}\right\|_{K} \quad\left(\text { where }\left\|f_{m}\right\|_{K}=\sup _{z \in K}\left|f_{m}(z)\right|\right)
$$

is continuous on $\mathscr{O}(D)$. Thus we see that $\mathscr{O}(D)$ becomes an absolutely $\mathbb{Z}_{+}$-graded Fréchet algebra (see Definition 5.22).

Proposition 6.4. Let $A$ be a $\widehat{\otimes}$-algebra, and let $\sigma$ be an endomorphism of $A$ such that the family $\left\{\sigma^{k}: k \in \mathbb{Z}_{+}\right\}$is equicontinuous. Then for each balanced domain $D \subseteq \mathbb{C}^{n}$ there exists a unique $\widehat{\otimes}$-algebra structure on $\mathscr{O}(D, A)$ such that the canonical embeddings

$$
\begin{aligned}
\mathscr{O}(D) & \hookrightarrow \mathscr{O}(D, A), \quad f \mapsto f \otimes 1, \\
A & \hookrightarrow \mathscr{O}(D, A), \quad a \mapsto 1 \otimes a,
\end{aligned}
$$

are algebra homomorphisms, and such that

$$
z_{i} a=\sigma(a) z_{i} \quad(a \in A, i=1, \ldots, n) .
$$

The resulting $\widehat{\otimes}$-algebra $\mathscr{O}(D, A ; \sigma)$ is equal to $A \widehat{\#}^{\mathbb{Z}}+\mathscr{O}(D)$. If, in addition, $A$ is an Arens-Michael algebra, then so is $\mathscr{O}(D, A ; \sigma)$. Finally, if $A$ is holomorphically finitely generated, then so is $\mathscr{O}(D, A ; \sigma)$.

Proof. We have an equicontinuous action of $\mathbb{Z}_{+}$on $A$ given by $k \cdot a=\sigma^{k}(a)(k \in$ $\mathbb{Z}_{+}, a \in A$ ). As we have already observed, $\mathscr{O}(D)$ is an absolutely $\mathbb{Z}_{+}$-graded Fréchet algebra. Now the result follows from Theorem 5.24 modulo the obvious fact that $A$ and $z_{1}, \ldots, z_{n}$ generate a dense subalgebra of $\mathscr{O}(D, A ; \sigma)$. 
Proposition 6.4 can be extended to the case of several commuting endomorphisms of $A$. To this end, we have to impose more restrictions on the domain $D$. Recall that a domain $D \subseteq \mathbb{C}^{n}$ is a Reinhardt domain if for each $z=\left(z_{1}, \ldots, z_{n}\right) \in D$ and each $\lambda=\left(\lambda_{1}, \ldots, \lambda_{n}\right) \in \mathbb{C}^{n}$ such that $\left|\lambda_{i}\right|=1(i=$ $1, \ldots, n)$ we have $\left(\lambda_{1} z_{1}, \ldots, \lambda_{n} z_{n}\right) \in D$. If the above condition holds for all $\lambda=\left(\lambda_{1}, \ldots, \lambda_{n}\right) \in \mathbb{C}^{n}$ such that $\left|\lambda_{i}\right| \leq 1(i=1, \ldots, n)$, then $D$ is a complete Reinhardt domain. Equivalently, $D$ is a Reinhardt domain (respectively, a complete Reinhardt domain) if and only if $D$ is a union of closed polyannuli (respectively, of closed polydisks) centered at 0 .

Given a Reinhardt domain $D \subseteq \mathbb{C}^{n}$, let

$$
N_{D}=\left\{j \in\{1, \ldots, n\}: D \cap\left\{z \in \mathbb{C}^{n}: z_{j}=0\right\} \neq \varnothing\right\} .
$$

Note that if $0 \in D$ (e.g., if $D$ is complete), then $N_{D}=\{1, \ldots, n\}$. Clearly, the monomial $z^{k}$ (where $k \in \mathbb{Z}^{n}$ ) is defined everywhere on $D$ if and only if $k_{j} \geq 0$ for all $j \in N_{D}$.

The following is essentially a restatement of a well-known fact from complex analysis (see, e.g., [41, Chap. II, §1.2]).

Lemma 6.5. For each Reinhardt domain $D \subseteq \mathbb{C}^{n}$, the subspaces

$$
\mathscr{O}(D)_{k}= \begin{cases}\operatorname{span}\left\{z^{k}\right\} & \text { if } k_{j} \geq 0 \text { for all } j \in N_{D} ; \quad\left(k \in \mathbb{Z}^{n}\right) \\ 0 & \text { otherwise }\end{cases}
$$

form an absolute $\mathbb{Z}^{n}$-grading on $\mathscr{O}(D)$. In particular, if $0 \in D$, then $\mathscr{O}(D)$ is absolutely $\mathbb{Z}_{+}^{n}$-graded.

Proof. By [41, Chap. II, §1.2, Theorem 1.5], each $f \in \mathscr{O}(D)$ has a unique Laurent series representation

$$
f(z)=\sum_{k \in \mathbb{Z}^{n}} c_{k} z^{k} \quad(z \in D),
$$

and $c_{k}=0$ whenever $k_{j}<0$ for some $j \in N_{D}$. Thus for each $k \in \mathbb{Z}^{n}$ we have $f_{k}=c_{k} z^{k} \in \mathscr{O}(D)_{k}$. Fix a compact polyannulus $\overline{\mathbb{D}}_{r, R}^{n} \subset D$, and let $\lambda \in(0,1)$ be such that $\overline{\mathbb{D}}_{\lambda r, \lambda^{-1} R} \subset D$. By [41, Chap. II, §1.2, (1.11)], we have

$$
\left\|f_{k}\right\|_{\overline{\mathbb{D}}_{r, R}} \leq \lambda^{|k|}\|f\|_{\overline{\mathbb{D}}_{\lambda r, \lambda-1}} \quad\left(k \in \mathbb{Z}^{n}\right) .
$$

Therefore

$$
\sum_{k \in \mathbb{Z}^{n}}\left\|f_{k}\right\|_{\overline{\mathbb{D}}_{r, R}} \leq C\|f\|_{\overline{\mathbb{D}}_{\lambda r, \lambda-1},}
$$

where

$$
C=\sum_{k \in \mathbb{Z}^{n}} \lambda^{|k|}=\left(\frac{1+\lambda}{1-\lambda}\right)^{n}
$$


Since each compact set $K \subset D$ is covered by finitely many compact polyannuli contained in $D$, it follows from (6.7) that the seminorm $\|f\|_{K}^{\prime \prime}=\sum_{k}\left\|f_{k}\right\|_{K}$ is continuous on $\mathscr{O}(D)$. This completes the proof.

Proposition 6.6. Let $A$ be a $\widehat{\otimes}$-algebra, and let $\sigma=\left(\sigma_{1}, \ldots, \sigma_{n}\right)$ be a commuting family of endomorphisms of $A$. Suppose that $D \subseteq \mathbb{C}^{n}$ is a Reinhardt domain, and that one of the following conditions holds:

(i) $0 \in D$, and the subsemigroup of $\operatorname{End}(A)$ generated by $\sigma_{1}, \ldots, \sigma_{n}$ is equicontinuous;

(ii) $\sigma_{1}, \ldots, \sigma_{n}$ are automorphisms, and the subgroup of $\operatorname{Aut}(A)$ generated by $\sigma_{1}, \ldots, \sigma_{n}$ is equicontinuous.

Then there exists a unique $\widehat{\otimes}$-algebra structure on $\mathscr{O}(D, A)$ such that the canonical embeddings

$$
\begin{aligned}
\mathscr{O}(D) & \hookrightarrow \mathscr{O}(D, A), \quad f \mapsto f \otimes 1, \\
A & \hookrightarrow \mathscr{O}(D, A), \quad a \mapsto 1 \otimes a,
\end{aligned}
$$

are algebra homomorphisms, and such that

$$
z_{i} a=\sigma_{i}(a) z_{i} \quad(a \in A, i=1, \ldots, n) .
$$

Let $S=\mathbb{Z}_{+}^{n}$ for case (i), and $S=\mathbb{Z}^{n}$ for case (ii). Then the resulting $\widehat{\otimes}$-algebra $\mathscr{O}(D, A ; \sigma)$ is equal to $A \widehat{\#}^{S} \mathscr{O}(D)$. If, in addition, $A$ is an Arens-Michael algebra, then so is $\mathscr{O}(D, A ; \sigma)$. Finally, if $A$ is holomorphically finitely generated, then so is $\mathscr{O}(D, A ; \sigma)$.

We omit the proof as it is similar to that of Proposition 6.4.

Remark 6.7. In practice, the equicontinuity of the subsemigroup of $\operatorname{End}(A)$ generated by commuting endomorphisms $\sigma_{1}, \ldots, \sigma_{n}$ is often reduced to the following property. Suppose that there exists a defining family $\left\{\|\cdot\|_{\lambda}: \lambda \in \Lambda\right\}$ of seminorms on $A$ such that

$$
\left\|\sigma_{i}(a)\right\|_{\lambda} \leq\|a\|_{\lambda} \quad(a \in A, \lambda \in \Lambda, i=1, \ldots, n) .
$$

Then for each $k \in \mathbb{Z}_{+}^{n}$ and each $a \in A$ we have $\left\|\sigma^{k}(a)\right\|_{\lambda} \leq\|a\|_{\lambda}$, which obviously implies that the subsemigroup of $\operatorname{End}(A)$ generated by $\sigma_{1}, \ldots, \sigma_{n}$ is equicontinuous. Similarly, if $\sigma_{1}, \ldots, \sigma_{n}$ are automorphisms, then the subgroup of $\operatorname{Aut}(A)$ generated by $\sigma_{1}, \ldots, \sigma_{n}$ is equicontinuous provided that there exists a defining family $\left\{\|\cdot\|_{\lambda}\right.$ : $\lambda \in \Lambda$ \} of seminorms on $A$ such that

$$
\left\|\sigma_{i}(a)\right\|_{\lambda}=\|a\|_{\lambda} \quad(a \in A, \lambda \in \Lambda, i=1, \ldots, n) .
$$

Remark 6.8. Algebras $\mathscr{O}(D, A ; \sigma)$ from Proposition 6.6 (i) can be viewed as analytic analogs of iterated Ore extensions of the following special form. Given 
an algebra $A$ and a commuting $n$-tuple $\sigma=\left(\sigma_{1}, \ldots, \sigma_{n}\right)$ of endomorphisms of $A$, we define a chain $A_{0} \subset \cdots \subset A_{n}$ of algebras by letting $A_{0}=A$ and $A_{k}=A_{k-1}\left[z_{k} ; \tilde{\sigma}_{k}\right]$, where $\tilde{\sigma}_{k}$ is the endomorphism of $A_{k-1}$ uniquely determined by $\left.\tilde{\sigma}_{k}\right|_{A}=\sigma_{k}$ and $\tilde{\sigma}_{k}\left(z_{i}\right)=z_{i}(i=1, \ldots, k-1)$. The existence of such an endomorphism easily follows from the commutativity of the $\sigma_{i}$ 's. We have $A_{n}=A\left[z_{1}, \ldots, z_{n}\right]$ with multiplication uniquely determined by (6.8) and by the requirement that the embeddings $A \hookrightarrow A_{n}$ and $\mathbb{C}\left[z_{1}, \ldots, z_{n}\right] \hookrightarrow A_{n}$ are algebra homomorphisms. If now $A, \sigma$, and $D$ are such as in Proposition 6.6 (i), then $A_{n}$ is obviously a dense subalgebra of $\mathscr{O}(D, A ; \sigma)$. If condition (ii) of Proposition 6.6 holds, and if $N_{D}=\varnothing$ (e.g., if $D$ is a polyannulus), then the situation is similar, but one should use iterated Laurent extensions instead of iterated Ore extensions.

In the special case where $D=\mathbb{D}_{R}^{n}$ is a polydisk in $\mathbb{C}^{n}$, the algebra $\mathscr{O}(D, A ; \sigma)$ can also be defined by a similar iterative procedure. Specifically, we have a chain $B_{0} \subset \cdots \subset B_{n}$ of Fréchet algebras with $B_{0}=A$ and $B_{k}=\mathscr{O}\left(\mathbb{D}_{R_{k}}, B_{k-1} ; \tilde{\sigma}_{k}\right)$, where

$$
\tilde{\sigma}_{k}=\sigma_{k} \otimes 1: A \widehat{\otimes} \mathscr{O}\left(\mathbb{D}_{R_{1}} \times \cdots \times \mathbb{D}_{R_{k-1}}\right) \rightarrow A \widehat{\otimes} \mathscr{O}\left(\mathbb{D}_{R_{1}} \times \cdots \times \mathbb{D}_{R_{k-1}}\right) .
$$

It is easy to show that $B_{n} \cong \mathscr{O}(D, A ; \sigma)$. A similar picture holds in the case where $D$ is a polyannulus.

\section{Examples}

7.1. Arens-Michael envelopes. Let $A$ be a finitely generated algebra, and suppose that $A$ is good enough to be interpreted as the algebra of "regular functions" on a "noncommutative affine variety". According to the point of view adopted in [38], the Arens-Michael envelope of $A$ is then a natural candidate for the algebra of "holomorphic functions" on the same "variety". This agrees with the philosophy of the present paper, as we will now see.

Proposition 7.1. If $A$ is a finitely generated algebra, then $\widehat{A}$ is holomorphically finitely generated.

Proof. Let $A=F_{n} / I$, where $I \subset F_{n}$ is a two-sided ideal. Applying [38, Corollary 3.2], we conclude that $\widehat{A}=\mathscr{F}_{n} / J$, where $J$ is the closure of $I$ in $\mathscr{F}_{n}$. By Proposition 3.20, $\widehat{A}$ is an HFG algebra.

We have already noticed in Section 2 that, according to [46], the Arens-Michael envelope of the polynomial algebra $\mathbb{C}\left[z_{1}, \ldots, z_{n}\right]$ is the algebra $\mathscr{O}\left(\mathbb{C}^{n}\right)$ of entire functions, while the Arens-Michael envelope of the free algebra $F_{n}$ is the algebra $\mathscr{F}_{n}$ of free entire functions. The following example is a slight generalization of [38, Example 3.6] 
Example 7.2. Let $\left(X, \mathscr{O}_{X}^{\mathrm{reg}}\right)$ be an affine scheme of finite type over $\mathbb{C}$, and let $\left(X_{h}, \mathscr{O}_{X_{h}}\right)$ be the complex space associated to $\left(X, \mathscr{O}_{X}^{\text {reg }}\right)$ (cf. [18, Appendix B]). We claim that the Arens-Michael envelope of $A=\mathscr{O}^{\mathrm{reg}}(X)$ is $\mathscr{O}\left(X_{h}\right)$. Indeed, we have $A=\mathscr{O}^{\text {reg }}\left(\mathbb{C}^{n}\right) / I$ for an ideal $I \subset \mathscr{O}^{\text {reg }}\left(\mathbb{C}^{n}\right)$. Let $f_{1}, \ldots, f_{q} \in \mathscr{O}^{\text {reg }}\left(\mathbb{C}^{n}\right)$ generate $I$; then, by definition [18, Appendix B], we have $\mathscr{O}_{X_{h}}=\mathscr{O}_{\mathbb{C}^{n}} / \mathscr{I}$, where $\mathscr{I} \subset \mathscr{O}_{\mathbb{C}^{n}}$ is the ideal sheaf generated by $f_{1}, \ldots, f_{q}$. Thus we have an exact sequence

$$
\mathscr{O}_{\mathbb{C}^{n}}^{q} \stackrel{\left(f_{1}, \ldots, f_{q}\right)}{\longrightarrow} \mathscr{O}_{\mathbb{C}^{n}} \rightarrow \mathscr{O}_{X_{h}} \rightarrow 0
$$

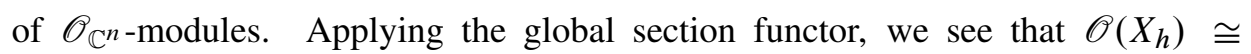
$\mathscr{O}\left(\mathbb{C}^{n}\right) / J$, where $J \subset \mathscr{O}\left(\mathbb{C}^{n}\right)$ is the ideal generated by $f_{1}, \ldots, f_{q}$ (note that $J$ is automatically closed in $\mathscr{O}\left(\mathbb{C}^{n}\right)$; see $[17$, V.6]). We claim that $J=\bar{I}$, the closure of $I$ in $\mathscr{O}\left(\mathbb{C}^{n}\right)$. Indeed, since $J$ is closed, we have $\bar{I} \subseteq J$. On the other hand, $\bar{I}$ is an ideal of $\mathscr{O}\left(\mathbb{C}^{n}\right)$, because $\mathscr{O}^{\text {reg }}\left(\mathbb{C}^{n}\right)$ is dense in $\mathscr{O}\left(\mathbb{C}^{n}\right)$. Since $J$ is algebraically generated by $f_{1}, \ldots, f_{q}$, we see that $J \subseteq \bar{I}$, and finally $J=\bar{I}$. By using the fact that the Arens-Michael functor commutes with quotients [38, Corollary 3.2], we conclude that

$$
\widehat{\mathscr{O}^{\text {reg }}(X)} \cong \widehat{\mathscr{O}^{\text {reg }}\left(\mathbb{C}^{n}\right)} / \bar{I} \cong \mathscr{O}\left(\mathbb{C}^{n}\right) / J \cong \mathscr{O}\left(X_{h}\right)
$$

Thus we may interpret the Arens-Michael functor as a noncommutative extension of the analytification functor that takes an affine scheme $\left(X, \mathscr{O}_{X}^{\text {reg }}\right)$ of finite type over $\mathbb{C}$ to the associated complex space $\left(X_{h}, \mathscr{O}_{X_{h}}\right)$.

Remark 7.3. The following example shows that a closed subalgebra of an HFG algebra need not be an HFG algebra. Let $\mathfrak{g}$ be the two-dimensional solvable Lie algebra with basis $\{x, y\}$ and commutation relation $[x, y]=y$. As was shown in [38, Proposition 5.2], the Arens-Michael envelope of the enveloping algebra $U(\mathfrak{g})$ is given by

$$
\widehat{U}(\mathfrak{g})=\left\{a=\sum_{i, j=0}^{\infty} c_{i j} x^{i} y^{j}:\|a\|_{n, t}=\sum_{i=0}^{\infty} \sum_{j=0}^{n}\left|c_{i j}\right| t^{i} \quad \forall t>0, \forall n \in \mathbb{N}\right\} .
$$

The topology on $\widehat{U}(\mathfrak{g})$ is given by the seminorms $\|\cdot\|_{n, t}(t>0, n \in \mathbb{N})$. By Proposition 7.1, $\widehat{U}(\mathfrak{g})$ is an HFG algebra. On the other hand, it immediate from (7.1) that the closed subalgebra of $\widehat{U}(\mathfrak{g})$ generated by $y$ is the algebra $\mathbb{C}[[y]]$ of formal power series. Clearly, $\mathbb{C}[[y]]$ is not isomorphic to $\mathscr{O}(X)$ for any Stein space $\left(X, \mathscr{O}_{X}\right)$ and hence is not an HFG algebra.

The following two examples [38] will motivate our further constructions, so we reproduce them here.

Example 7.4. Let $\mathbf{q}=\left(q_{i j}\right)$ be a complex $n \times n$-matrix such that $q_{i i}=1$ and $q_{j i}=q_{i j}^{-1}$ for all $i, j=1, \ldots, n$ (such matrices are called multiplicatively 
antisymmetric). Recall that the algebra $\mathscr{O}_{\mathbf{q}}^{\text {reg }}\left(\mathbb{C}^{n}\right)$ of regular functions on the quantum affine $n$-space is generated by $n$ elements $x_{1}, \ldots, x_{n}$ subject to the relations $x_{i} x_{j}=q_{i j} x_{j} x_{i}$ for all $i, j$ (see, e.g., [2]). If $q_{i j}=1$ for all $i, j$, then $\mathscr{O}_{\mathbf{q}}^{\text {reg }}\left(\mathbb{C}^{n}\right)$ is nothing but the polynomial algebra $\mathbb{C}\left[x_{1}, \ldots, x_{n}\right]=\mathscr{O}^{\text {reg }}\left(\mathbb{C}^{n}\right)$. Of course, $\mathscr{O}_{\mathbf{q}}^{\text {reg }}\left(\mathbb{C}^{n}\right)$ is noncommutative unless $q_{i j}=1$ for all $i, j$, but the monomials $x^{k}=x_{1}^{k_{1}} \cdots x_{n}^{k_{n}}\left(k \in \mathbb{Z}_{+}^{n}\right)$ still form a basis of $\mathscr{O}_{\mathbf{q}}^{\text {reg }}\left(\mathbb{C}^{n}\right)$. Thus $\mathscr{O}_{\mathbf{q}}^{\text {reg }}\left(\mathbb{C}^{n}\right)$ may be viewed as a "deformed" polynomial algebra.

The Arens-Michael envelope of $\mathscr{O}_{\mathbf{q}}^{\text {reg }}\left(\mathbb{C}^{n}\right)$ is denoted by $\mathscr{O}_{\mathbf{q}}\left(\mathbb{C}^{n}\right)$ and is called the algebra of holomorphic functions on the quantum affine $n$-space. If $q_{i j}=1$ for all $i, j$, then $\mathscr{O}_{\mathbf{q}}\left(\mathbb{C}^{n}\right) \cong \mathscr{O}\left(\mathbb{C}^{n}\right)$ (see Section 2 or Example 7.2). The algebra $\mathscr{O}_{\mathbf{q}}\left(\mathbb{C}^{n}\right)$ has the following explicit description [38]. Given $d \in \mathbb{Z}_{+}$, let us identify each element $\alpha=\left(\alpha_{1}, \ldots, \alpha_{d}\right) \in W_{n, d}$ with the function $\alpha:\{1, \ldots, d\} \rightarrow\{1, \ldots, n\}, \alpha(i)=\alpha_{i}$. The symmetric group $S_{d}$ acts on $W_{n, d}$ via $\sigma(\alpha)=\alpha \sigma^{-1}\left(\alpha \in W_{n, d}, \sigma \in S_{d}\right)$. Clearly, for each $\alpha \in W_{n, d}$ and $\sigma \in S_{d}$ there exists a unique $\lambda(\sigma, \alpha) \in \mathbb{C}^{\times}$(where $\mathbb{C}^{\times}=\mathbb{C} \backslash\{0\}$ ) such that

$$
x_{\alpha}=\lambda(\sigma, \alpha) x_{\sigma(\alpha)} .
$$

Given $k=\left(k_{1}, \ldots, k_{n}\right) \in \mathbb{Z}_{+}^{n}$, let

$$
\delta(k)=(\underbrace{1, \ldots, 1}_{k_{1}}, \ldots, \underbrace{n, \ldots, n}_{k_{n}}) \in W_{n,|k|} .
$$

Define a weight function $w_{\mathbf{q}}: \mathbb{Z}_{+}^{n} \rightarrow \mathbb{R}_{+}$by

$$
w_{\mathbf{q}}(k)=\min \left\{|\lambda(\sigma, \delta(k))|: \sigma \in S_{|k|}\right\} .
$$

For example [38, Proposition 5.12], if $\left|q_{i j}\right| \geq 1$ whenever $i<j$, then $w_{\mathbf{q}}(k) \equiv 1$, and if $\left|q_{i j}\right| \leq 1$ whenever $i<j$, then $w_{\mathbf{q}}(k)=\prod_{i<j} q_{i j}^{k_{i} k_{j}}$. In particular, in the single-parameter case (i.e., in the case where $q_{i j}=q$ for all $i<j$ ) we have

$$
w_{q}(k)= \begin{cases}1 & \text { if }|q| \geq 1, \\ |q|^{\sum_{i<j} k_{i} k_{j}} & \text { if }|q|<1 .\end{cases}
$$

As was shown in [38, Theorem 5.11], for each multiplicatively antisymmetric complex $n \times n$-matrix $\mathbf{q}$ we have

$$
\mathscr{O}_{\mathbf{q}}\left(\mathbb{C}^{n}\right)=\left\{a=\sum_{k \in \mathbb{Z}_{+}^{n}} c_{k} x^{k}:\|a\|_{t}=\sum_{k \in \mathbb{Z}_{+}^{n}}\left|c_{k}\right| w_{\mathbf{q}}(k) t^{|k|}<\infty \forall t>0\right\} .
$$

The topology on $\mathscr{O}_{\mathbf{q}}\left(\mathbb{C}^{n}\right)$ is given by the norms $\|\cdot\|_{t}(t>0)$. Moreover, each norm $\|\cdot\|_{t}$ is submultiplicative.

Example 7.5. As in the previous example, let $\mathbf{q}=\left(q_{i j}\right)$ be a multiplicatively antisymmetric complex $n \times n$-matrix. Recall that the algebra $\mathscr{O}_{\mathbf{q}}^{\text {reg }}\left(\left(\mathbb{C}^{\times}\right)^{n}\right)$ of regular 
functions on the quantum affine $n$-torus is generated by $2 n$ elements $x_{1}^{ \pm 1}, \ldots, x_{n}^{ \pm 1}$ subject to the relations $x_{i} x_{j}=q_{i j} x_{j} x_{i}$ for all $i, j$, and $x_{i} x_{i}^{-1}=x_{i} x_{i}^{-1}=1$ for all $i$ (see, e.g., [2]). If $q_{i j}=1$ for all $i, j$, then $\mathscr{O}_{\mathbf{q}}^{\text {reg }}\left(\left(\mathbb{C}^{\times}\right)^{n}\right)$ is nothing but the Laurent polynomial algebra $\mathbb{C}\left[x_{1}^{ \pm 1}, \ldots, x_{n}^{ \pm 1}\right]=\mathscr{O}^{\text {reg }}\left(\left(\mathbb{C}^{\times}\right)^{n}\right)$. In the general case, the Laurent monomials $x^{k}=x_{1}^{k_{1}} \cdots x_{n}^{k_{n}}\left(k \in \mathbb{Z}^{n}\right)$ form a basis of $\mathscr{O}_{\mathbf{q}}^{\mathrm{reg}}\left(\left(\mathbb{C}^{\times}\right)^{n}\right)$, and so $\mathscr{O}_{\mathbf{q}}^{\text {reg }}\left(\left(\mathbb{C}^{\times}\right)^{n}\right)$ may be viewed as a "deformed" Laurent polynomial algebra.

Assume now that $\left|q_{i j}\right|=1$ for all $i, j$. The Arens-Michael envelope of $\mathscr{O}_{\mathbf{q}}^{\text {reg }}\left(\left(\mathbb{C}^{\times}\right)^{n}\right)$ is denoted by $\mathscr{O}_{\mathbf{q}}\left(\left(\mathbb{C}^{\times}\right)^{n}\right)$ and is called the algebra of holomorphic functions on the quantum affine $n$-torus. If $q_{i j}=1$ for all $i, j$, then $\mathscr{O}_{\mathbf{q}}\left(\left(\mathbb{C}^{\times}\right)^{n}\right) \cong$ $\mathscr{O}\left(\left(\mathbb{C}^{\times}\right)^{n}\right)$ (see Example 7.2). As was shown in [38, Corollary 5.22], we have

$$
\mathscr{O}_{\mathbf{q}}\left(\left(\mathbb{C}^{\times}\right)^{n}\right)=\left\{a=\sum_{k \in \mathbb{Z}^{n}} c_{k} x^{k}:\|a\|_{t}=\sum_{k \in \mathbb{Z}^{n}}\left|c_{k}\right| t^{|k|}<\infty \forall t>1\right\} .
$$

The topology on $\mathscr{O}_{\mathbf{q}}\left(\left(\mathbb{C}^{\times}\right)^{n}\right)$ is given by the norms $\|\cdot\|_{t}(t>1)$. Moreover, each norm $\|\cdot\|_{t}$ is submultiplicative. Note that, if $\left|q_{i j}\right| \neq 1$ for at least one pair of indices $i, j$, then the Arens-Michael envelope of $\mathscr{O}_{\mathbf{q}}^{\text {reg }}\left(\left(\mathbb{C}^{\times}\right)^{n}\right)$ is trivial [38, Proposition 5.23].

We refer to $[9,11,38]$ for explicit descriptions of Arens-Michael envelopes of some other finitely generated algebras, including quantum Weyl algebras, the algebra of quantum $2 \times 2$-matrices, and universal enveloping algebras. Note that, in many concrete cases, the Arens-Michael envelope of a "deformed polynomial algebra" can be interpreted as a "deformed power series algebra", similarly to (7.5) and (7.6).

7.2. Free polydisk. The following definition is motivated by (4.10).

Definition 7.6. We define the algebra of holomorphic functions on the free $n$ dimensional polydisk of polyradius $R=\left(R_{1}, \ldots, R_{n}\right) \in(0,+\infty]^{n}$ to be

$$
\mathscr{F}\left(\mathbb{D}_{R}^{n}\right)=\mathscr{O}\left(\mathbb{D}_{R_{1}}\right) \widehat{*} \cdots \widehat{*} \mathscr{O}\left(\mathbb{D}_{R_{n}}\right) .
$$

By Corollary 4.7, $\mathscr{F}\left(\mathbb{D}_{R}^{n}\right)$ is an HFG algebra. Letting $R_{i}=\infty$ for all $i$ and using (4.10), we see that $\mathscr{F}\left(\mathbb{C}^{n}\right)=\mathscr{F}_{n}$. Note that replacing in (4.10) and (7.7) the Arens-Michael free product $\widehat{*}$ by the projective tensor product $\widehat{\otimes}$ yields the algebras of holomorphic functions on $\mathbb{C}^{n}$ and $\mathbb{D}_{R}^{n}$, respectively.

We have the canonical "restriction" map $\mathscr{F}_{n} \rightarrow \mathscr{F}\left(\mathbb{D}_{R}^{n}\right)$ defined to be the free product of the restriction maps $\mathscr{O}(\mathbb{C}) \rightarrow \mathscr{O}\left(\mathbb{D}_{R_{i}}\right)(i=1, \ldots, n)$. For each $i=$ $1, \ldots, n$, the canonical image of the free generator $\zeta_{i} \in \mathscr{F}_{n}$ in $\mathscr{F}\left(\mathbb{D}_{R}^{n}\right)$ will also be denoted by $\zeta_{i}$. The next result shows that $\mathscr{F}\left(\mathbb{D}_{R}^{n}\right)$ has a universal property similar to (2.5). In what follows, given an algebra $A$ and an element $a \in A$, the spectrum of $a$ in $A$ will be denoted by $\sigma_{A}(a)$.

Proposition 7.7. Let $A$ be an Arens-Michael algebra, and let $a=\left(a_{1}, \ldots, a_{n}\right)$ be an $n$-tuple in $A^{n}$ such that $\sigma_{A}\left(a_{i}\right) \subseteq \mathbb{D}_{R_{i}}$ for all $i=1, \ldots, n$. Then there exists 
a unique continuous homomorphism $\gamma_{a}^{\text {free }}: \mathscr{F}\left(\mathbb{D}_{R}^{n}\right) \rightarrow$ A such that $\gamma_{a}^{\text {free }}\left(\zeta_{i}\right)=a_{i}$ for all $i=1, \ldots, n$. Moreover, the assignment $a \mapsto \gamma_{a}^{\text {free }}$ determines a natural isomorphism

$$
\operatorname{Hom}_{\mathrm{AM}}\left(\mathscr{F}\left(\mathbb{D}_{R}^{n}\right), A\right) \cong\left\{a \in A^{n}: \sigma_{A}\left(a_{i}\right) \subseteq \mathbb{D}_{R_{i}} \forall i=1, \ldots, n\right\} \quad(A \in \mathrm{AM}) .
$$

Proof. For $n=1$, the above result is standard (see, e.g., [28, VI.3, Theorem 3.2]). Hence for each $n \in \mathbb{N}$ we have natural isomorphisms

$$
\begin{aligned}
\left.\operatorname{Hom}_{\mathrm{AM}}\left(\mathscr{F}\left(\mathbb{D}_{R}^{n}\right), A\right)\right) & \cong \prod_{i=1}^{n} \operatorname{Hom}_{\mathrm{AM}}\left(\mathscr{O}\left(\mathbb{D}_{R_{i}}\right), A\right) \\
& \cong\left\{a \in A^{n}: \sigma_{A}\left(a_{i}\right) \subseteq \mathbb{D}_{R_{i}} \forall i=1, \ldots, n\right\} .
\end{aligned}
$$

The algebra $\mathscr{F}\left(\mathbb{D}_{R}^{n}\right)$ can also be described more explicitly as follows. Given $d \geq 2$ and $\alpha=\left(\alpha_{1}, \ldots, \alpha_{d}\right) \in W_{n}$, let $s(\alpha)$ denote the cardinality of the set

$$
\left\{i \in\{1, \ldots, d-1\}: \alpha_{i} \neq \alpha_{i+1}\right\} .
$$

If $|\alpha| \in\{0,1\}$, we set $s(\alpha)=|\alpha|-1$. Let also $(0, R)=\prod_{i=1}^{n}\left(0, R_{i}\right)$.

Proposition 7.8. We have

$$
\begin{aligned}
& \mathscr{F}\left(\mathbb{D}_{R}^{n}\right)= \\
& \left\{a=\sum_{\alpha \in W_{n}} c_{\alpha} \zeta_{\alpha}:\|a\|_{\rho, \tau}=\sum_{\alpha \in W_{n}}\left|c_{\alpha}\right| \rho_{\alpha} \tau^{s(\alpha)+1}<\infty \forall \rho \in(0, R), \forall \tau \geq 1\right\} .
\end{aligned}
$$

The topology on $\mathscr{F}\left(\mathbb{D}_{R}^{n}\right)$ is given by the norms $\|\cdot\|_{\rho, \tau}(\rho \in(0, R), \tau \geq 1)$, and the multiplication is given by concatenation. Moreover, each norm $\|\cdot\|_{\rho, \tau}$ is submultiplicative.

Proof. Let $I=\{1, \ldots, n\}$, and let $A_{i}=\mathscr{O}\left(\mathbb{D}_{R_{i}}\right)$ for all $i \in I$. Note that $A_{i}=$ $\mathbb{C} 1_{A_{i}} \oplus A_{i}^{\circ}$, where $A_{i}^{\circ}=\left\{f \in A_{i}: f(0)=0\right\}$. Hence Corollary 4.4 applies, and (4.8) holds. We have

$$
A_{i}^{\circ} \cong\left\{f=\sum_{k=1}^{\infty} c_{k} \zeta_{i}^{k}:\|f\|_{\rho_{i}}=\sum_{k=1}^{\infty}\left|c_{k}\right| \rho_{i}^{k}<\infty \forall \rho_{i} \in\left(0, R_{i}\right)\right\} .
$$

Recall the standard fact that the projective tensor product of two Köthe sequence spaces is again a Köthe sequence space (cf. [24, 41.7]). Hence for each $\alpha=$ $\left(\alpha_{1}, \ldots, \alpha_{d}\right) \in I_{\infty}$ with $d>0$ we have

$$
A_{\alpha} \cong\left\{u=\sum_{k \in \mathbb{N}^{d}} c_{k} \zeta_{\alpha_{1}}^{k_{1}} \cdots \zeta_{\alpha_{d}}^{k_{d}}:\|u\|_{\rho}^{(\alpha)}=\sum_{k \in \mathbb{N}^{d}}\left|c_{k}\right| \rho_{\alpha_{1}}^{k_{1}} \cdots \rho_{\alpha_{d}}^{k_{d}}<\infty \forall \rho \in(0, R)\right\} .
$$


Now (4.8) yields

$$
\begin{aligned}
& \mathscr{F}\left(\mathbb{D}_{R}^{n}\right) \cong\left\{a=\lambda 1+\sum_{d=1}^{\infty} \sum_{\alpha \in I_{d}} \sum_{k \in \mathbb{N}^{d}} c_{\alpha k} \zeta_{\alpha_{1}}^{k_{1}} \cdots \zeta_{\alpha_{d}}^{k_{d}}: \lambda \in \mathbb{C},\right. \\
& \left.\|a\|_{\rho, \tau}=|\lambda|+\sum_{d=1}^{\infty} \sum_{\alpha \in I_{d}} \sum_{k \in \mathbb{N}^{d}}\left|c_{\alpha k}\right| \rho_{\alpha_{1}}^{k_{1}} \cdots \rho_{\alpha_{d}}^{k_{d}} \tau^{d}<\infty \forall \rho \in(0, R), \quad \forall \tau \geq 1\right\} .
\end{aligned}
$$

Let $W_{n}^{+}=\bigsqcup_{d \geq 1} W_{n, d}$, and let $S=\bigsqcup_{d \geq 1}\left(I_{d} \times \mathbb{N}^{d}\right)$. We have a bijection between $S$ and $W_{n}^{+}$given by

$$
\begin{aligned}
(\alpha, k)=\left(\left(\alpha_{1}, \ldots, \alpha_{d}\right),\right. & \left.\left(k_{1}, \ldots, k_{d}\right)\right) \in I_{d} \times \mathbb{N}^{d} \\
& \mapsto \beta(\alpha)=(\underbrace{\alpha_{1}, \ldots, \alpha_{1}}_{k_{1}}, \ldots, \underbrace{\alpha_{d}, \ldots, \alpha_{d}}_{k_{d}}) \in W_{n}^{+} .
\end{aligned}
$$

Note that $|\beta(\alpha)|=|k|$ and that $|\alpha|=s(\beta(\alpha))+1$. Using (7.9) and (7.8), we obtain

$$
\begin{aligned}
\mathscr{F}\left(\mathbb{D}_{R}^{n}\right) \cong\left\{a=\lambda 1+\sum_{\beta \in W_{n}^{+}} c_{\beta} \zeta_{\beta}: \lambda \in \mathbb{C},\right. \\
\left.\|a\|_{\rho, \tau}=|\lambda|+\sum_{\beta \in W_{n}^{+}}\left|c_{\beta}\right| \rho_{\beta} \tau^{s(\beta)+1}<\infty \forall \rho \in(0, R), \forall \tau \geq 1\right\} \\
\cong\left\{a=\sum_{\beta \in W_{n}} c_{\beta} \zeta_{\beta}:\|a\|_{\rho, \tau}=\sum_{\beta \in W_{n}}\left|c_{\beta}\right| \rho_{\beta} \tau^{s(\beta)+1}<\infty \forall \rho \in(0, R), \forall \tau \geq 1\right\} .
\end{aligned}
$$

The submultiplicativity of $\|\cdot\|_{\rho, \tau}$ follows from Corollary 4.4.

Remark 7.9. Another natural candidate for the algebra of holomorphic functions on the free polydisk was introduced by J. L. Taylor $[46,47]$. By definition,

$$
\mathscr{F}^{T}\left(\mathbb{D}_{R}^{n}\right)=\left\{a=\sum_{\alpha \in W_{n}} c_{\alpha} \zeta_{\alpha}:\|a\|_{\rho}=\sum_{\alpha \in W_{n}}\left|c_{\alpha}\right| \rho_{\alpha}<\infty \forall \rho \in(0, R)\right\} .
$$

Obviously, $\mathscr{F}\left(\mathbb{D}_{R}^{n}\right) \subseteq \mathscr{F}^{T}\left(\mathbb{D}_{R}^{n}\right)$, and $\|a\|_{\rho} \leq\|a\|_{\rho, \tau}$ for each $a \in \mathscr{F}\left(\mathbb{D}_{R}^{n}\right)$, each $\rho \in(0, R)$, and each $\tau \geq 1$. Hence the embedding of $\mathscr{F}\left(\mathbb{D}_{R}^{n}\right)$ into $\mathscr{F}^{T}\left(\mathbb{D}_{R}^{n}\right)$ is continuous. For $n=1$, we clearly have $\mathscr{F}^{T}\left(\mathbb{D}_{R}^{1}\right)=\mathscr{F}\left(\mathbb{D}_{R}^{1}\right)=\mathscr{O}\left(\mathbb{D}_{R}^{1}\right)$. To compare $\mathscr{F}^{T}\left(\mathbb{D}_{R}^{n}\right)$ with $\mathscr{F}\left(\mathbb{D}_{R}^{n}\right)$ for $n \geq 2$, it is convenient to consider the following three cases.

(i) Suppose that $R_{i}=\infty$ for all $i$, i.e., $\mathbb{D}_{R}^{n}=\mathbb{C}^{n}$. Comparing (2.3) and (7.10), we see that $\mathscr{F}^{T}\left(\mathbb{C}^{n}\right)=\mathscr{F}_{n}=\mathscr{F}\left(\mathbb{C}^{n}\right)$, both algebraically and topologically.

(ii) Suppose that $R_{i}<\infty$ for at most one $i \in\{1, \ldots, n\}$. We claim that $\mathscr{F}^{T}\left(\mathbb{D}_{R}^{n}\right)=\mathscr{F}\left(\mathbb{D}_{R}^{n}\right)$ in this case. Without loss of generality, assume that 
$R_{1}<\infty$ and $R_{2}=\ldots=R_{n}=\infty$. Given $\rho \in(0, R)$ and $\tau \geq 1$, let $\rho^{\prime}=\left(\rho_{1}, \tau^{2} \rho_{2}, \ldots, \tau^{2} \rho_{n}\right)$. We clearly have $\rho^{\prime} \in(0, R)$. To prove the claim, it suffices to show that for each $\alpha \in W_{n}$ we have

$$
\left\|\zeta_{\alpha}\right\|_{\rho, \tau} \leq \tau\left\|\zeta_{\alpha}\right\|_{\rho^{\prime}}
$$

Fix $\alpha \in W_{n}$ and write $\zeta_{\alpha}$ in the form

$$
\zeta_{\alpha}=\zeta_{1}^{m_{1}} \zeta_{\alpha(1)} \zeta_{1}^{m_{2}} \zeta_{\alpha(2)} \cdots \zeta_{1}^{m_{k}} \zeta_{\alpha(k)} \zeta_{1}^{m_{k+1}},
$$

where $m_{1}, \ldots, m_{k+1} \in \mathbb{Z}_{+}, \alpha(1), \ldots, \alpha(k) \in W_{n}$ do not contain 1 , and $|\alpha(i)| \geq 1$ for all $i=1, \ldots, k$. We clearly have

$$
s(\alpha) \leq 2 k+\sum_{i=1}^{k} s(\alpha(i)) .
$$

Since for each $\beta \in W_{n}$ we have $s(\beta)+1 \leq|\beta|$, it follows that

$$
s(\alpha) \leq 2 k+\sum_{i=1}^{k} s(\alpha(i)) \leq k+\sum_{i=1}^{k}|\alpha(i)| \leq 2 \sum_{i=1}^{k}|\alpha(i)| .
$$

Therefore

$$
\left\|\zeta_{\alpha}\right\|_{\rho, \tau}=\rho_{\alpha} \tau^{s(\alpha)+1} \leq \tau \rho_{\alpha} \tau^{2 \sum|\alpha(i)|},
$$

while

$$
\left\|\zeta_{\alpha}\right\|_{\rho^{\prime}}=\rho_{1}^{\sum m_{j}} \tau^{2|\alpha(1)|} \rho_{\alpha(1)} \cdots \tau^{2|\alpha(k)|} \rho_{\alpha(k)}=\rho_{\alpha} \tau^{2 \sum|\alpha(i)|} .
$$

Comparing (7.12) with (7.13), we obtain (7.11), as required. Therefore $\mathscr{F}^{T}\left(\mathbb{D}_{R}^{n}\right)=$ $\mathscr{F}\left(\mathbb{D}_{R}^{n}\right)$, both algebraically and topologically.

(iii) Finally, suppose that $R_{i}<\infty$ for at least two $i \in\{1, \ldots, n\}$. As was shown in $[25,46], \mathscr{F}^{T}\left(\mathbb{D}_{R}^{n}\right)$ is not nuclear in this case. Hence $\mathscr{F}^{T}\left(\mathbb{D}_{R}^{n}\right)$ is not an HFG algebra, and so $\mathscr{F}^{T}\left(\mathbb{D}_{R}^{n}\right) \neq \mathscr{F}\left(\mathbb{D}_{R}^{n}\right)$. Moreover, the topology on $\mathscr{F}\left(\mathbb{D}_{R}^{n}\right)$ is strictly stronger than that inherited from $\mathscr{F}^{T}\left(\mathbb{D}_{R}^{n}\right)$.

7.3. $q$-products of balanced and Reinhardt domains. In the remaining subsections, we will introduce deformed products on some algebras of holomorphic functions, and we will show that the resulting deformed algebras are HFG algebras. Let us start with the simplest case of the algebra $\mathscr{O}\left(D_{1} \times D_{2}\right)$, where $D_{1} \subseteq \mathbb{C}^{m}$ and $D_{2} \subseteq \mathbb{C}^{n}$ are balanced domains.

Proposition 7.10. Let $D_{1} \subseteq \mathbb{C}^{m}$ and $D_{2} \subseteq \mathbb{C}^{n}$ be balanced domains. Denote by $z_{1}, \ldots, z_{m}$ (respectively, $\left.w_{1}, \ldots, w_{n}\right)$ the coordinates on $\mathbb{C}^{m}$ (respectively, $\left.\mathbb{C}^{n}\right)$. 
Then for each $q \in \mathbb{C}^{\times}$there exists a unique continuous multiplication on $\mathscr{O}\left(D_{1} \times D_{2}\right)$ such that

$$
\begin{aligned}
z_{i} z_{j} & =z_{j} z_{i} & & (i, j=1, \ldots, m), \\
w_{i} w_{j} & =w_{j} w_{i} & & (i, j=1, \ldots, n), \\
z_{i} w_{j} & =q w_{j} z_{i} & & (i=1, \ldots, m, j=1, \ldots, n) .
\end{aligned}
$$

The resulting Fréchet algebra $\mathscr{O}\left(D_{1} \times_{q} D_{2}\right)$ is an HFG algebra.

Proof. By interchanging $D_{1}$ and $D_{2}$ if necessary, we can assume that $|q| \geq 1$. Consider the endomorphism $\sigma$ of $\mathscr{O}\left(D_{1}\right)$ given by $(\sigma f)(z)=f\left(q^{-1} z\right)$. For each compact set $K \subset D_{1}$ and each $f \in \mathscr{O}\left(D_{1}\right)$ with homogeneous expansion $f=$ $\sum_{m \in \mathbb{Z}_{+}} f_{m}$, we have

$$
\|\sigma(f)\|_{K}^{\prime}=\sum_{m \in \mathbb{Z}_{+}}\left\|\sigma\left(f_{m}\right)\right\|_{K}=\sum_{m \in \mathbb{Z}_{+}}|q|^{-m}\left\|f_{m}\right\|_{K} \leq \sum_{m \in \mathbb{Z}_{+}}\left\|f_{m}\right\|_{K}=\|f\|_{K}^{\prime} .
$$

Hence (6.9) holds, and the subsemigroup of $\operatorname{End}\left(\mathscr{O}\left(D_{1}\right)\right)$ generated by $\sigma$ is equicontinuous. Now Proposition 6.4 yields the HFG algebra $\mathscr{O}\left(D_{2}, \mathscr{O}\left(D_{1}\right) ; \sigma\right)$. Identifying $\mathscr{O}\left(D_{2}, \mathscr{O}\left(D_{1}\right) ; \sigma\right)$ with $\mathscr{O}\left(D_{1} \times D_{2}\right)$ as a Fréchet space (see Section 2), we see that the relations (7.14) hold in $\mathscr{O}\left(D_{2}, \mathscr{O}\left(D_{1}\right) ; \sigma\right)$. Letting $\mathscr{O}\left(D_{1} \times_{q} D_{2}\right)=$ $\mathscr{O}\left(D_{2}, \mathscr{O}\left(D_{1}\right) ; \sigma\right)$, we obtain the required algebra.

If we assume that $D_{1}$ and $D_{2}$ are Reinhardt domains, then we can generalize the commutation relations (7.14) as follows.

Proposition 7.11. Let $D_{1} \subseteq \mathbb{C}^{m}$ and $D_{2} \subseteq \mathbb{C}^{n}$ be Reinhardt domains. Denote by $z_{1}, \ldots, z_{m}$ (respectively, $\left.w_{1}, \ldots, w_{n}\right)$ the coordinates on $\mathbb{C}^{m}$ (respectively, $\mathbb{C}^{n}$ ). Let $\mathbf{q}=\left(q_{i j}\right)$ be a complex $m \times n$-matrix, and assume that one of the following conditions holds:

(i) $D_{1}$ and $D_{2}$ are complete, and either $\left|q_{i j}\right| \geq 1$ for all $i, j$, or $0<\left|q_{i j}\right| \leq 1$ for all $i, j$;

(ii) $\left|q_{i j}\right|=1$ for all $i, j$.

Then there exists a unique continuous multiplication on $\mathscr{O}\left(D_{1} \times D_{2}\right)$ such that

$$
\begin{aligned}
z_{i} z_{j} & =z_{j} z_{i} & & (i, j=1, \ldots, m), \\
w_{i} w_{j} & =w_{j} w_{i} & & (i, j=1, \ldots, n), \\
z_{i} w_{j} & =q_{i j} w_{j} z_{i} & & (i=1, \ldots, m, j=1, \ldots, n) .
\end{aligned}
$$

The resulting Fréchet algebra $\mathscr{O}\left(D_{1} \times_{\mathbf{q}} D_{2}\right)$ is an $H F G$ algebra.

Proof.

(i) By interchanging $D_{1}$ and $D_{2}$ if necessary, we can assume that $\left|q_{i j}\right| \geq 1$ for all $i, j$. Consider the commuting endomorphisms $\sigma_{1}, \ldots, \sigma_{n}$ of $\mathscr{O}\left(D_{1}\right)$ given 
by $\left(\sigma_{j} f\right)(z)=f\left(q_{1 j}^{-1} z_{1}, \ldots, q_{m j}^{-1} z_{m}\right)$. For each compact set $K \subset D_{1}$ and each $f \in \mathscr{O}\left(D_{1}\right)$ with Taylor expansion $f=\sum_{k \in \mathbb{Z}_{+}^{m}} c_{k} z^{k}$, we have

$$
\left\|\sigma_{j}(f)\right\|_{K}^{\prime \prime}=\sum_{k \in \mathbb{Z}_{+}^{m}}\left|c_{k}\right|\left\|\sigma_{j}\left(z^{k}\right)\right\|_{K} \leq \sum_{k \in \mathbb{Z}_{+}^{m}}\left|c_{k}\right|\left\|z^{k}\right\|_{K}=\|f\|_{K}^{\prime \prime} .
$$

Hence (6.9) holds, and the subsemigroup of $\operatorname{End}\left(\mathscr{O}\left(D_{1}\right)\right)$ generated by $\sigma_{1}, \ldots, \sigma_{n}$ is equicontinuous. Now Proposition 6.6 (i) yields the HFG algebra $\mathscr{O}\left(D_{2}, \mathscr{O}\left(D_{1}\right) ; \sigma\right)$. Identifying $\mathscr{O}\left(D_{2}, \mathscr{O}\left(D_{1}\right) ; \sigma\right)$ with $\mathscr{O}\left(D_{1} \times D_{2}\right)$ as a Fréchet space (see Section 2), we see that the relations (7.15) hold in $\mathscr{O}\left(D_{2}, \mathscr{O}\left(D_{1}\right) ; \sigma\right)$. Letting $\mathscr{O}\left(D_{1} \times \mathbf{q} D_{2}\right)=$ $\mathscr{O}\left(D_{2}, \mathscr{O}\left(D_{1}\right) ; \sigma\right)$, we obtain the required algebra.

(ii) The proof is similar to (i), the only difference is that now $\sigma_{1}, \ldots, \sigma_{n}$ are automorphisms satisfying (6.10), and so the subgroup of $\operatorname{Aut}\left(\mathscr{O}\left(D_{1}\right)\right)$ generated by $\sigma_{1}, \ldots, \sigma_{n}$ is equicontinuous.

7.4. Quantum polydisk. Our next example is motivated by (7.5). Let $\mathbf{q}=\left(q_{i j}\right)$ be a multiplicatively antisymmetric complex $n \times n$-matrix. Observe that, for each $t>0$, the norm $\|\cdot\|_{t}$ on $\mathscr{O}_{\mathbf{q}}^{\text {reg }}\left(\mathbb{C}^{n}\right)$ given by (7.5) is a special case of the following one. Given $\rho \in(0,+\infty)^{n}$ and $a=\sum_{k} c_{k} x^{k} \in \mathscr{O}_{\mathbf{q}}^{\text {reg }}\left(\mathbb{C}^{n}\right)$, let

$$
\|a\|_{\rho}=\sum_{k \in \mathbb{Z}_{+}^{n}}\left|c_{k}\right| w_{\mathbf{q}}(k) \rho^{k},
$$

where the function $w_{\mathbf{q}}: \mathbb{Z}_{+}^{n} \rightarrow \mathbb{R}_{+}$is given by (7.3). The same argument as in [38, Lemma 5.10] shows that $\|\cdot\|_{\rho}$ is submultiplicative.

Definition 7.12. Let $R \in(0,+\infty]^{n}$. We define the algebra of holomorphic functions on the quantum n-polydisk of polyradius $R$ by

$$
\mathscr{O}_{\mathbf{q}}\left(\mathbb{D}_{R}^{n}\right)=\left\{a=\sum_{k \in \mathbb{Z}_{+}^{n}} c_{k} x^{k}:\|a\|_{\rho}=\sum_{k \in \mathbb{Z}_{+}^{n}}\left|c_{k}\right| w_{\mathbf{q}}(k) \rho^{k}<\infty \forall \rho \in(0, R)\right\} .
$$

The topology on $\mathscr{O}_{\mathbf{q}}\left(\mathbb{D}_{R}^{n}\right)$ is given by the norms $\|\cdot\|_{\rho}(\rho \in(0, R))$, and the multiplication on $\mathscr{O}_{\mathbf{q}}\left(\mathbb{D}_{R}^{n}\right)$ is uniquely determined by $x_{i} x_{j}=q_{i j} x_{j} x_{i}$ for all $i, j$.

In other words, $\mathscr{O}_{\mathbf{q}}\left(\mathbb{D}_{R}^{n}\right)$ is the completion of $\mathscr{O}_{\mathbf{q}}^{\text {reg }}\left(\mathbb{C}^{n}\right)$ with respect to the family $\left\{\|\cdot\|_{\rho}: \rho \in(0, R)\right\}$ of submultiplicative norms. Clearly, if $q_{i j}=1$ for all $i, j$, then $\mathscr{O}_{\mathbf{q}}\left(\mathbb{D}_{R}^{n}\right)$ is topologically isomorphic to the algebra $\mathscr{O}\left(\mathbb{D}_{R}^{n}\right)$ of holomorphic functions on the polydisk $\mathbb{D}_{R}^{n}$.

Theorem 7.13. There exists a unique continuous homomorphism

$$
\pi: \mathscr{F}\left(\mathbb{D}_{R}^{n}\right) \rightarrow \mathscr{O}_{\mathbf{q}}\left(\mathbb{D}_{R}^{n}\right) \quad \text { such that } \quad \pi\left(\zeta_{i}\right)=x_{i} \quad(i=1, \ldots, n) .
$$

Moreover, $\pi$ is onto, and $\operatorname{Ker} \pi$ coincides with the closed two-sided ideal of $\mathscr{F}\left(\mathbb{D}_{R}^{n}\right)$ generated by the elements $\zeta_{i} \zeta_{j}-q_{i j} \zeta_{j} \zeta_{i}$ for all $i, j=1, \ldots, n$. Finally, Ker $\pi$ is a complemented subspace of $\mathscr{F}\left(\mathbb{D}_{R}^{n}\right)$. 
To prove Theorem 7.13, we need some preparation. Given $k \in \mathbb{Z}_{+}^{n}$, let

$$
W(k)=\left\{\sigma(\delta(k)): \sigma \in S_{|k|},|\lambda(\sigma, \delta(k))|=w_{\mathbf{q}}(k)\right\}
$$

(for notation, see Example 7.4). Observe that, if $\alpha \in W_{n}$ and $\sigma_{1}, \sigma_{2} \in S_{|\alpha|}$ are such that $\sigma_{1}(\alpha)=\sigma_{2}(\alpha)$, then $\lambda\left(\sigma_{1}, \alpha\right)=\lambda\left(\sigma_{2}, \alpha\right)$ (see (7.2)). In other words, $\lambda(\sigma, \alpha)$ depends only on $\alpha$ and $\sigma(\alpha)$. As a consequence we obtain the following.

Lemma 7.14. If $\sigma \in S_{|k|}$, then $\sigma(\delta(k)) \in W(k)$ if and only if $|\lambda(\sigma, \delta(k))|=w_{\mathbf{q}}(k)$.

Recall [38, Lemma 5.8] that, for each $\alpha \in W_{n}$ and each $\sigma, \tau \in S_{|\alpha|}$ we have

$$
\lambda(\sigma \tau, \alpha)=\lambda(\sigma, \tau(\alpha)) \lambda(\tau, \alpha) .
$$

Lemma 7.15. If $\alpha \in W(k)$, then

(i) $|\lambda(\sigma, \alpha)| \geq 1$ for each $\sigma \in S_{|k|}$;

(ii) $|\lambda(\sigma, \alpha)|=1$ if and only if $\sigma(\alpha) \in W(k)$.

Proof. Choose $\tau \in S_{|k|}$ such that $\alpha=\tau(\delta(k))$. By Lemma 7.14, we have $|\lambda(\tau, \delta(k))|=w_{\mathbf{q}}(k)$. Moreover, $\sigma(\alpha) \in W(k)$ if and only if $|\lambda(\sigma \tau, \delta(k))|=w_{\mathbf{q}}(k)$. Using (7.16), we see that

$$
w_{\mathbf{q}}(k) \leq|\lambda(\sigma \tau, \delta(k))|=|\lambda(\sigma, \alpha)| w_{\mathbf{q}}(k) .
$$

This readily implies both (i) and (ii).

Let $d \in \mathbb{Z}_{+}$, and let $d_{1}, \ldots, d_{p} \in \mathbb{Z}_{+}$be such that $d_{1}+\cdots+d_{p}=d$. We will identify each $p$-tuple $\left(\beta_{1}, \ldots, \beta_{p}\right)$ such that $\beta_{i} \in W_{n, d_{i}}(i=1, \ldots, p)$ with the element of $W_{n, d}$ obtained from $\beta_{1}, \ldots, \beta_{p}$ by concatenation.

Definition 7.16. Let $\ell, d \in \mathbb{Z}_{+}, \ell \leq d$. We say that $\beta \in W_{n, \ell}$ is a subword of $\alpha \in W_{n, d}$ if there exist $\ell^{\prime}, \ell^{\prime \prime} \in \mathbb{Z}_{+}$and $\beta^{\prime} \in W_{n, \ell^{\prime}}, \beta^{\prime \prime} \in W_{n, \ell^{\prime \prime}}$ such that $d=\ell^{\prime}+\ell+\ell^{\prime \prime}$ and $\alpha=\left(\beta^{\prime}, \beta, \beta^{\prime \prime}\right)$.

Definition 7.17. Given $j \in\{1, \ldots, n\}$, a $j$-word is a word of the form $(j, \ldots, j) \in$ $W_{n, d}$ for some $d \in \mathbb{Z}_{+}$.

Definition 7.18. Let $\alpha \in W_{n, d}$, and let $\beta$ be a $j$-subword of $\alpha$. We say that $\beta$ is a maximal $j$-subword of $\alpha$ if $\beta$ is not a proper subword any other $j$-subword of $\alpha$.

Given $\alpha \in W_{n, d}$ and $j \in\{1, \ldots, n\}$, let $N(\alpha, j)$ denote the number of maximal nonempty $j$-subwords of $\alpha$. Let also

$$
\begin{aligned}
\mathbf{n c}(\alpha) & =\{j \in\{1, \ldots, n\}: N(\alpha, j) \geq 2\}, \\
\mathbf{c}(\alpha) & =\{1, \ldots, n\} \backslash \mathbf{n c}(\alpha) .
\end{aligned}
$$


Definition 7.19. We say that $\alpha \in W_{n, d}$ is compact if $\mathbf{n c}(\alpha)=\varnothing$. Equivalently, $\alpha$ is compact if there exist $\sigma \in S_{n}$ and $k=\left(k_{1}, \ldots, k_{n}\right) \in \mathbb{Z}_{+}^{n}$ such that

$$
\alpha=(\underbrace{\sigma(1), \ldots, \sigma(1)}_{k_{1}}, \ldots, \underbrace{\sigma(n), \ldots, \sigma(n)}_{k_{n}}) .
$$

Given $s, t, d \in \mathbb{Z}_{+}$such that $1 \leq s<t \leq d$, consider the cycle

$$
\sigma_{s, t}=(s s+1 \ldots t-1 t) \in S_{d} .
$$

Let also $\sigma_{t, s}=\sigma_{s, t}^{-1}$. We clearly have

$$
\begin{aligned}
& \sigma_{s, t}=(s s+1)(s+1 s+2) \cdots(t-1 t), \\
& \sigma_{t, s}=(t-1 t)(t-2 t-1) \cdots(s s+1) .
\end{aligned}
$$

Lemma 7.20. For each $\alpha \in W_{n, d}$ we have

$$
\lambda\left(\sigma_{s, t}, \alpha\right)=\prod_{i=s}^{t-1} q_{\alpha_{i} \alpha_{t}}, \quad \lambda\left(\sigma_{t, s}, \alpha\right)=\prod_{i=s+1}^{t} q_{\alpha_{s} \alpha_{i}} .
$$

Proof. We prove only the first equality, the proof of the second being similar. We use induction on $p=t-s$. For $p=1$, there is nothing to prove. Let now $p \geq 2$, and let $\alpha^{\prime}=(t-1 t) \alpha$. Since $\sigma_{s, t}=\sigma_{s, t-1}(t-1 t)$, the induction hypothesis and (7.16) yield

$$
\begin{aligned}
\lambda\left(\sigma_{s, t}, \alpha\right) & =\lambda\left(\sigma_{s, t-1}, \alpha^{\prime}\right) \lambda((t-1 t), \alpha) \\
& =\left(\prod_{i=s}^{t-2} q_{\alpha_{i}^{\prime} \alpha_{t-1}^{\prime}}\right) q_{\alpha_{t-1} \alpha_{t}}=\left(\prod_{i=s}^{t-2} q_{\alpha_{i} \alpha_{t}}\right) q_{\alpha_{t-1} \alpha_{t}}=\prod_{i=s}^{t-1} q_{\alpha_{i} \alpha_{t} .} .
\end{aligned}
$$

Lemma 7.21. Let $k \in \mathbb{Z}_{+}^{n}$. For each noncompact $\alpha \in W(k)$ and each $j \in \mathbf{n c}(\alpha)$ there exists $\sigma \in S_{|k|}$ such that $\sigma(\alpha) \in W(k), N(\sigma(\alpha), j)=N(\alpha, j)-1$, and $\mathbf{c}(\alpha) \subseteq \mathbf{c}(\sigma(\alpha))$.

Proof. Since $j \in \mathbf{n c}(\alpha)$, we can write $\alpha$ in the form

$$
\alpha=\left(\beta_{1}, \gamma_{1}, \beta_{2}, \gamma_{2}, \beta_{3}\right)
$$

where $\gamma_{1}$ and $\gamma_{2}$ are maximal nonempty $j$-subwords of $\alpha$, and $\beta_{1}, \beta_{2}, \beta_{3}$ are subwords of $\alpha$ such that $\beta_{2} \neq \varnothing$. Let also

$$
r=\left|\beta_{1}\right|, \quad s=\left|\beta_{1}\right|+\left|\gamma_{1}\right|, \quad t=\left|\beta_{1}\right|+\left|\gamma_{1}\right|+\left|\beta_{2}\right| .
$$

In particular, we have $\alpha_{s}=\alpha_{t+1}=j$. Using (7.17), we obtain

$$
\lambda\left(\sigma_{t, s}, \alpha\right)=\prod_{i=s+1}^{t} q_{j \alpha_{i}}, \quad \lambda\left(\sigma_{s+1, t+1}, \alpha\right)=\prod_{i=s+1}^{t} q_{\alpha_{i} j} .
$$


Hence $\lambda\left(\sigma_{s+1, t+1}, \alpha\right)=\lambda\left(\sigma_{t, s}, \alpha\right)^{-1}$. Applying Lemma 7.15, we conclude that $\left|\lambda\left(\sigma_{t, s}, \alpha\right)\right|=\left|\lambda\left(\sigma_{s+1, t+1}, \alpha\right)\right|=1$, whence $\sigma_{t, s}(\alpha) \in W(k)$.

Let $\alpha^{\prime}=\sigma_{t, s}(\alpha)$. We have

$$
\alpha^{\prime}=\left(\beta_{1}, \gamma_{1}^{\prime}, \beta_{2}, \gamma_{2}^{\prime}, \beta_{3}\right),
$$

where $\gamma_{2}^{\prime}=\left(j, \gamma_{2}\right)$ and $\gamma_{1}=\left(\gamma_{1}^{\prime}, j\right)$. If $\gamma_{1}^{\prime} \neq \varnothing$, then the same procedure applied to $\alpha^{\prime}$ and to the maximal nonempty $j$-subwords $\gamma_{1}^{\prime}, \gamma_{2}^{\prime}$ yields

$$
\alpha^{\prime \prime}=\sigma_{t-1, s-1}\left(\alpha^{\prime}\right)=\left(\beta_{1}, \gamma_{1}^{\prime \prime}, \beta_{2}, \gamma_{2}^{\prime \prime}, \beta_{3}\right) \in W(k),
$$

where $\gamma_{2}^{\prime \prime}=\left(j, \gamma_{2}^{\prime}\right)$ and $\gamma_{1}^{\prime}=\left(\gamma_{1}^{\prime \prime}, j\right)$. After finitely many steps we obtain

$$
\alpha^{(s-r)}=\sigma_{t-s+r+1, r+1}\left(\alpha^{(s-r-1)}\right)=\left(\beta_{1}, \beta_{2}, \gamma_{1}, \gamma_{2}, \beta_{3}\right) \in W(k) .
$$

We clearly have $\alpha^{(s-r)}=\sigma(\alpha)$, where $\sigma=\prod_{i=1}^{s-r} \sigma_{t-s+r+i, r+i}$. Comparing (7.19) with (7.18), we conclude that $N\left(\alpha^{(s-r)}, j\right)=N(\alpha, j)-1$ and $\mathbf{c}(\alpha) \subseteq \mathbf{c}\left(\alpha^{(s-r)}\right)$.

Lemma 7.22. For each $k \in \mathbb{Z}_{+}^{n}, W(k)$ contains a compact word.

Proof. Choose $\alpha \in W(k)$ such that $|\mathbf{c}(\alpha)| \geq|\mathbf{c}(\beta)|$ for each $\beta \in W(k)$. Assume, towards a contradiction, that $\alpha$ is noncompact, and fix any $j \in \mathbf{n c}(\alpha)$. Applying Lemma 7.21 finitely many times, we obtain $\beta \in W(k)$ such that $N(\beta, j)=1$ and $\mathbf{c}(\alpha) \subsetneq \mathbf{c}(\beta)$. Thus $|\mathbf{c}(\beta)|>|\mathbf{c}(\alpha)|$. The resulting contradiction completes the proof.

Proof of Theorem 7.13. Fix $i \in\{1, \ldots, n\}$, and let $z_{i} \in \mathscr{O}\left(\mathbb{D}_{R_{i}}\right)$ denote the complex coordinate. Let $e_{i} \in \mathbb{Z}_{+}^{n}$ denote the $n$-tuple with 1 at the $i$ th position, 0 elsewhere. Clearly, for each $d \in \mathbb{Z}_{+}$the element $\delta\left(d e_{i}\right)=(i, \ldots, i) \in W_{n, d}$ is invariant under the action of $S_{d}$. Hence $w_{\mathbf{q}}\left(d e_{i}\right)=1$, and

$$
\left\|x_{i}^{d}\right\|_{\rho}=\left\|x^{d e_{i}}\right\|_{\rho}=\rho_{i}^{d}=\left\|z_{i}^{d}\right\|_{\rho_{i}} \quad(\rho \in(0, R)) .
$$

This implies that there exists a Fréchet algebra embedding $\mathscr{O}\left(\mathbb{D}_{R_{i}}\right) \hookrightarrow \mathscr{O}_{\mathbf{q}}\left(\mathbb{D}_{R}^{n}\right)$ that sends the complex coordinate $z_{i}$ to $x_{i}$. Hence, by Definition 7.6, there exists a unique continuous homomorphism

$$
\pi: \mathscr{F}\left(\mathbb{D}_{R}^{n}\right) \rightarrow \mathscr{O}_{\mathbf{q}}\left(\mathbb{D}_{R}^{n}\right), \quad \zeta_{i} \mapsto x_{i} \quad(i=1, \ldots, n) .
$$

Let us construct a continuous linear map

$$
\varkappa: \mathscr{O}_{\mathbf{q}}\left(\mathbb{D}_{R}^{n}\right) \rightarrow \mathscr{F}\left(\mathbb{D}_{R}^{n}\right) \quad \text { such that } \quad \pi \varkappa=1 .
$$

To this end, fix any $k \in \mathbb{Z}_{+}^{n}$ and choose a compact word $\alpha_{k} \in W(k)$ (see Lemma 7.22). Let $\sigma_{k} \in S_{|k|}$ be such that $\alpha_{k}=\sigma_{k}(\delta(k))$. Then

$$
x^{k}=x_{\delta(k)}=\lambda\left(\sigma_{k}, \delta(k)\right) x_{\alpha_{k}}=\pi\left(\lambda\left(\sigma_{k}, \delta(k)\right) \zeta_{\alpha_{k}}\right) .
$$


We claim that for each $a=\sum_{k \in \mathbb{Z}_{+}^{n}} c_{k} x^{k} \in \mathscr{O}_{\mathbf{q}}\left(\mathbb{D}_{R}^{n}\right)$ the series

$$
\sum_{k \in \mathbb{Z}_{+}^{n}} c_{k} \lambda\left(\sigma_{k}, \delta(k)\right) \zeta_{\alpha_{k}}
$$

absolutely converges in $\mathscr{F}\left(\mathbb{D}_{R}^{n}\right)$. Indeed, since $\alpha_{k}$ is compact, it follows that $s\left(\alpha_{k}\right) \leq$ $n-1$ for each $k \in \mathbb{Z}_{+}^{n}$. By Lemma 7.14, we also have $\left|\lambda\left(\sigma_{k}, \delta(k)\right)\right|=w_{\mathbf{q}}(k)$. Hence for each $\rho \in(0, R)$ and each $\tau \geq 1$ we obtain

$$
\sum_{k \in \mathbb{Z}_{+}^{n}}\left\|c_{k} \lambda\left(\sigma_{k}, \delta(k)\right) \zeta_{\alpha_{k}}\right\|_{\rho, \tau} \leq \sum_{k \in \mathbb{Z}_{+}^{n}}\left|c_{k}\right| w_{\mathbf{q}}(k) \rho^{k} \tau^{n}=\tau^{n}\|a\|_{\rho} .
$$

Therefore (7.22) converges to an element $\varkappa(a) \in \mathscr{F}\left(\mathbb{D}_{R}^{n}\right)$. It is also immediate from (7.23) that for each $a \in \mathscr{O}_{\mathbf{q}}\left(\mathbb{D}_{R}^{n}\right)$ we have $\|\mathcal{x}(a)\|_{\rho, \tau} \leq \tau^{n}\|a\|_{\rho}$, whence $\varkappa: \mathscr{O}_{\mathbf{q}}\left(\mathbb{D}_{R}^{n}\right) \rightarrow \mathscr{F}\left(\mathbb{D}_{R}^{n}\right)$ is a continuous linear map. Using $(7.21)$, we see that $\pi \varkappa=1$. Hence $\pi$ is onto, and $\operatorname{Ker} \pi$ is a complemented subspace of $\mathscr{F}\left(\mathbb{D}_{R}^{n}\right)$.

Let now $I \subset \mathscr{F}\left(\mathbb{D}_{R}^{n}\right)$ denote the closed two-sided ideal generated by the elements $\zeta_{i} \zeta_{j}-q_{i j} \zeta_{j} \zeta_{i}$ for all $i, j$. Clearly, $I \subseteq \operatorname{Ker} \pi$, and hence $\pi$ induces a continuous homomorphism

$$
\bar{\pi}: \mathscr{F}\left(\mathbb{D}_{R}^{n}\right) / I \rightarrow \mathscr{O}_{\mathbf{q}}\left(\mathbb{D}_{R}^{n}\right), \quad \bar{\zeta}_{i} \mapsto x_{i} \quad(i=1, \ldots, n),
$$

where $\bar{\zeta}_{i}=\zeta_{i}+I \in \mathscr{F}\left(\mathbb{D}_{R}^{n}\right) / I$. Let $\bar{\varkappa}: \mathscr{O}_{\mathbf{q}}\left(\mathbb{D}_{R}^{n}\right) \rightarrow \mathscr{F}\left(\mathbb{D}_{R}^{n}\right) / I$ denote the composition of $\varkappa$ with the quotient map $\mathscr{F}\left(\mathbb{D}_{R}^{n}\right) \rightarrow \mathscr{F}\left(\mathbb{D}_{R}^{n}\right) / I$. It is immediate from (7.20) that $\bar{\pi} \bar{x}=1$. On the other hand, it follows from the definition of $I$ that the linear span of the set $\left\{\bar{\zeta}_{\alpha_{k}}: k \in \mathbb{Z}_{+}^{n}\right\}$ is dense in $\mathscr{F}\left(\mathbb{D}_{R}^{n}\right) / I$. Hence $\operatorname{Im} \bar{x}$ is also dense in $\mathscr{F}\left(\mathbb{D}_{R}^{n}\right) / I$. Together with $\bar{\pi} \overline{\mathcal{X}}=1$, this implies that $\bar{\pi}$ and $\overline{\mathcal{X}}$ are topological isomorphisms. Therefore $I=\operatorname{Ker} \pi$. This completes the proof.

Corollary 7.23. $\mathscr{O}_{\mathbf{q}}\left(\mathbb{D}_{R}^{n}\right)$ is an HFG algebra.

Proof. Immediate from Theorem 7.13, Corollary 4.7, and Proposition 3.18.

7.5. Quantum polyannulus. Our last example is motivated by (7.6). Let $\mathbf{q}=\left(q_{i j}\right)$ be a multiplicatively antisymmetric complex $n \times n$-matrix such that $\left|q_{i j}\right|=1$ for all $i, j$. For each $k \in \mathbb{Z}^{n}$, define $k^{+}, k^{-} \in \mathbb{Z}^{n}$ by $k_{i}^{+}=\max \left\{k_{i}, 0\right\}$ and $k_{i}^{-}=$ $\min \left\{k_{i}, 0\right\}$. Given $r, R \in[0,+\infty]^{n}$, we write $r<R$ if $r_{i}<R_{i}$ for all $i$. In this case, let $(r, R)=\prod_{i=1}^{n}\left(r_{i}, R_{i}\right)$. Given $\rho, \tau \in(0,+\infty)^{n}$ with $\rho<\tau$ and $a=\sum_{k} c_{k} x^{k} \in \mathscr{O}_{\mathbf{q}}^{\text {reg }}\left(\left(\mathbb{C}^{\times}\right)^{n}\right)$, let

$$
\|a\|_{\rho, \tau}=\sum_{k \in \mathbb{Z}^{n}}\left|c_{k}\right| \rho^{k^{-}} \tau^{k^{+}}
$$

Clearly, $\|\cdot\|_{\rho, \tau}$ is a norm on $\mathscr{O}_{\mathbf{q}}^{\mathrm{reg}}\left(\left(\mathbb{C}^{\times}\right)^{n}\right)$. Observe that for each $k \in \mathbb{Z}^{n}$ we have

$$
\left\|x^{k}\right\|_{\rho, \tau}=\rho^{k^{-}} \tau^{k^{+}}=\max \left\{\left|z^{k}\right|: z \in \overline{\mathbb{D}}_{\rho, \tau}^{n}\right\} .
$$


Since $\left|q_{i j}\right|=1$ for all $i, j$, it follows that

$$
\left\|x^{k} x^{\ell}\right\|_{\rho, \tau}=\left\|x^{k+\ell}\right\|_{\rho, \tau} \leq\left\|x^{k}\right\|_{\rho, \tau}\left\|x^{\ell}\right\|_{\rho, \tau},
$$

whence the norm $\|\cdot\|_{\rho, \tau}$ is submultiplicative on $\mathscr{O}_{\mathbf{q}}^{\text {reg }}\left(\left(\mathbb{C}^{\times}\right)^{n}\right)$. Observe that, for each $t>1$, the norm $\|\cdot\|_{t}$ given by (7.6) is a special case of the above construction, since

$$
\|\cdot\|_{t}=\|\cdot\|_{\left(t^{-1}, \ldots, t^{-1}\right),(t, \ldots, t)} .
$$

Definition 7.24. Let $r, R \in[0,+\infty]^{n}, \quad r<R$. We define the algebra of holomorphic functions on the quantum n-polyannulus of polyradii $r$ and $R$ by

$$
\begin{aligned}
\mathscr{O}_{\mathbf{q}}\left(\mathbb{D}_{r, R}^{n}\right)=\left\{a=\sum_{k \in \mathbb{Z}^{n}} c_{k} x^{k}\right. & :\|a\|_{\rho, \tau} \\
& \left.=\sum_{k \in \mathbb{Z}^{n}}\left|c_{k}\right| \rho^{k^{-}} \tau^{k^{+}}<\infty \forall \rho, \tau \in(r, R), \rho<\tau\right\} .
\end{aligned}
$$

The topology on $\mathscr{O}_{\mathbf{q}}\left(\mathbb{D}_{r, R}^{n}\right)$ is given by the norms $\|\cdot\|_{\rho, \tau}(\rho, \tau \in(r, R), \rho<\tau)$, and the multiplication on $\mathscr{O}_{\mathbf{q}}\left(\mathbb{D}_{r, R}^{n}\right)$ is uniquely determined by $x_{i} x_{j}=q_{i j} x_{j} x_{i}$ for all $i, j$.

In other words, $\mathscr{O}_{\mathbf{q}}\left(\mathbb{D}_{r, R}^{n}\right)$ is the completion of $\mathscr{O}_{\mathbf{q}}^{\text {reg }}\left(\left(\mathbb{C}^{\times}\right)^{n}\right)$ with respect to the family $\left\{\|\cdot\|_{\rho, \tau}: \rho, \tau \in(r, R), \rho<\tau\right\}$ of submultiplicative norms. Observe that, as a Fréchet space, $\mathscr{O}_{\mathbf{q}}\left(\mathbb{D}_{r, R}^{n}\right)$ is topologically isomorphic to the space $\mathscr{O}\left(\mathbb{D}_{r, R}^{n}\right)$ of holomorphic functions on the polyannulus $\mathbb{D}_{r, R}^{n}$. Explicitly, the isomorphism takes each holomorphic function on $\mathbb{D}_{r, R}^{n}$ to its Laurent expansion about 0 . Clearly, if $q_{i j}=1$ for all $i, j$, then the above isomorphism is a Fréchet algebra isomorphism.

Theorem 7.25. $\mathscr{O}_{\mathbf{q}}\left(\mathbb{D}_{r, R}^{n}\right)$ is an HFG algebra.

Proof. For $n=1$, there is nothing to prove. Let $n \geq 2$, and assume that the result holds for $n-1$. Let $r^{\prime}=\left(r_{1}, \ldots, r_{n-1}\right), R^{\prime}=\left(R_{1}, \ldots, R_{n-1}\right)$, and $\mathbf{q}^{\prime}=\left(q_{i j}\right)_{i, j \leq n-1}$. Let $\sigma$ be the automorphism of $\mathscr{O}_{\mathbf{q}^{\prime}}^{\text {reg }}\left(\left(\mathbb{C}^{\times}\right)^{n-1}\right)$ given by $\sigma\left(x_{i}\right)=q_{i n}^{-1} x_{i}(i=1, \ldots, n-1)$. Clearly, for each $a \in \mathscr{O}_{\mathbf{q}^{\prime}}^{\mathrm{reg}}\left(\left(\mathbb{C}^{\times}\right)^{n-1}\right)$ we have

$$
\|\sigma(a)\|_{\rho, \tau}=\|a\|_{\rho, \tau} \quad\left(\rho, \tau \in\left(r^{\prime}, R^{\prime}\right), \rho<\tau\right) .
$$

Hence $\sigma$ uniquely extends to a topological automorphism of $\mathscr{O}_{\mathbf{q}^{\prime}}\left(\mathbb{D}_{r^{\prime}, R^{\prime}}^{n-1}\right)$. Moreover, (7.24) holds for each $a \in \mathscr{O}_{\mathbf{q}^{\prime}}\left(\mathbb{D}_{r^{\prime}, R^{\prime}}^{n-1}\right)$. Hence $\sigma$ satisfies (6.10), and the subgroup of $\operatorname{Aut}\left(\mathscr{O}_{\mathbf{q}^{\prime}}\left(\mathbb{D}_{r^{\prime}, R^{\prime}}^{n-1}\right)\right)$ generated by $\sigma$ is equicontinuous. Applying Proposition 6.6 (ii), we obtain the HFG algebra $\mathscr{O}\left(\mathbb{D}_{r_{n}, R_{n}}, \mathscr{O}_{\mathbf{q}^{\prime}}\left(\mathbb{D}_{r^{\prime}, R^{\prime}}^{n-1}\right) ; \sigma\right)$, which is easily seen to be topologically isomorphic to $\mathscr{O}_{\mathbf{q}}\left(\mathbb{D}_{r, R}^{n}\right)$.

Remark 7.26. If $\left|q_{i j}\right| \neq 1$ for at least one pair of indices $i, j$, then the only submultiplicative seminorm on $\mathscr{O}_{\mathbf{q}}^{\text {reg }}\left(\left(\mathbb{C}^{\times}\right)^{n}\right)$ is identically zero (see Example 7.5). That is why we make no attempt to define $\mathscr{O}_{\mathbf{q}}\left(\mathbb{D}_{r, R}^{n}\right)$ in this case. 
Remark 7.27. If $\mathbf{q}=\left(q_{i j}\right)$ is a multiplicatively antisymmetric complex $n \times n$-matrix satisfying $\left|q_{i j}\right| \geq 1$ for all $i<j$, then Corollary 7.23 can be proved similarly to Theorem 7.25. In the general case, however, the endomorphism $\sigma$ of $\mathscr{O}_{\mathbf{q}^{\prime}}^{\mathrm{reg}}\left(\mathbb{C}^{n-1}\right)$ given by $\sigma\left(x_{i}\right)=q_{i n}^{-1} x_{i}(i=1, \ldots, n-1)$ need not extend to a continuous endomorphism of $\mathscr{O}_{\mathbf{q}^{\prime}}\left(\mathbb{D}_{R^{\prime}}^{n-1}\right)$. Consider, for example, the simplest case where $n=2$, $R^{\prime}=1$, and $|q|=\left|q_{12}\right|<1$. We have $\mathscr{O}_{\mathbf{q}^{\prime}}^{\text {reg }}\left(\mathbb{C}^{n-1}\right)=\mathbb{C}\left[x_{1}\right], \mathscr{O}_{\mathbf{q}^{\prime}}\left(\mathbb{D}_{R^{\prime}}^{n-1}\right)=\mathscr{O}\left(\mathbb{D}_{1}\right)$, and $\sigma: \mathbb{C}\left[x_{1}\right] \rightarrow \mathbb{C}\left[x_{1}\right]$ acts by $\sigma\left(x_{1}\right)=q^{-1} x_{1}$. If $\sigma$ were continuous with respect to the topology inherited from $\mathscr{O}\left(\mathbb{D}_{1}\right)$, then for each $\rho \in(0,1)$ there would exist $r \in(\rho, 1)$ and $C>0$ such that $\|\sigma(a)\|_{\rho} \leq C\|a\|_{r}$ for each $a \in \mathbb{C}\left[x_{1}\right]$. For $a=x_{1}^{k}$, this yields $|q|^{-k} \rho^{k} \leq C r^{k}$, or, equivalently, $|q|^{-1} \leq C^{1 / k}(r / \rho)$. Letting first $k \rightarrow \infty$ and then $\rho \rightarrow 1$, we obtain $|q|^{-1} \leq 1$. The resulting contradiction shows that $\sigma$ is not continuous. Thus the above method cannot be applied to Corollary 7.23 in the general case.

\section{References}

[1] E. Beggs and S. P. Smith, Non-commutative complex differential geometry, J. Geom. Phys. 72 (2013), 7-33. Zbl 1286.32008 MR 3073899

[2] K. A. Brown and K. R. Goodearl, Lectures on algebraic quantum groups. Advanced Courses in Mathematics. CRM Barcelona. Birkhäuser Verlag, Basel, 2002. Zbl 1027.17010 MR 1898492

[3] A. Connes, Noncommutative Geometry. Academic Press, Inc., San Diego, CA, 1994. Zbl 0818.46076 MR 1303779

[4] J. Cuntz, Bivariante K-Theorie für lokalkonvexe Algebren und der Chern-ConnesCharakter. Doc. Math. 2 (1997), 139-182. Zbl 0920.19004 MR 1456322

[5] S. Dăscălescu, C. Năstăsescu, and Ş. Raianu, Hopf algebras. An introduction. Monographs and Textbooks in Pure and Applied Mathematics, 235. Marcel Dekker, Inc., New York, 2001. Zbl 0962.16026 MR 1786197

[6] S. Dineen, Complex analysis on infinite-dimensional spaces. Springer Monographs in Mathematics. Springer-Verlag London, Ltd., London, 1999. Zbl 1034.46504 MR 1705327

[7] A. A. Dosiev, Holomorphic functions of a basis of a nilpotent Lie algebra (Russian). Funktsional. Anal. i Prilozhen. 34 (2000), no. 4, 82-84; translation in Funct. Anal. Appl. 34 (2000), no. 4, 302-304. Zbl 0980.47017 MR 1819709

[8] A. A. Dosiev, Algebras of power series of elements of a Lie algebra, and Stodkowski spectra (Russian). Zap. Nauchn. Sem. S.-Peterburg. Otdel. Mat. Inst. Steklov. (POMI) 290 (2002), Issled. po Linein. Oper. i Teor. Funkts. 30, 72-121, 179; translation in J. Math. Sci. (N. Y.) 124 (2004), no. 2, 4886-4908. Zbl 1084.46040 MR 1942538

[9] A. A. Dosiev, Homological dimensions of the algebra of entire functions of elements of a nilpotent Lie algebra (Russian). Funktsional. Anal. i Prilozhen. 37 (2003), no. 1, 73-77; translation in Funct. Anal. Appl. 37 (2003), no. 1, 61-64. Zbl 1029.46065 MR 1988010 
[10] A. Dosiev, Local left invertibility for operator tuples and noncommutative localizations. J. K-Theory 4 (2009), no. 1, 163-191. Zbl 1185.46048 MR 2538721

[11] A. A. Dosi (Dosiev), Noncommutative holomorphic functions in elements of a Lie algebra and the absolute basis problem (Russian). Izv. Ross. Akad. Nauk Ser. Mat. 73 (2009), no. 6, 77-100; translation in Izv. Math. 73 (2009), no. 6, 1149-1171. Zbl 1209.46025 MR 2640979

[12] O. Forster, Primärzerlegung in Steinschen Algebren. Math. Ann. 154 (1964), 307-329. Zbl 0135.12602 MR 165387

[13] O. Forster, Zur Theorie der Steinschen Algebren und Moduln, Math. Z. 97 (1967), 376405. Zbl 0148.32203 MR 213611

[14] J. Gamst and K. Hoechsmann, Quaternions généralisés. C. R. Acad. Sci. Paris Sér. A-B 269 1969, A560-A562. Zbl 0197.03202 MR 252425

[15] J. M. Gracia-Bondía, J. C. Várilly, and H. Figueroa, Elements of Noncommutative Geometry. Birkhäuser Boston, Inc., Boston, MA, 2001. Zbl 0958.46039 MR 1789831

[16] A. Grothendieck, Produits tensoriels topologiques et espaces nucléaires, Mem. Amer. Math. Soc. 1955, no. 16, 140 pp. Zbl 0064.35501 MR 75539

[17] H. Grauert and R. Remmert, Theory of Stein Spaces. Springer-Verlag, Berlin-New York, 1979. Zbl 0433.32007 MR 580152

[18] R. Hartshorne, Algebraic Geometry. Graduate Texts in Mathematics, No. 52. SpringerVerlag, New York-Heidelberg, 1977. Zbl 0367.14001 MR 463157

[19] A. Ya. Helemskii, The homology of Banach and topological algebras. Kluwer Academic Publishers Group, Dordrecht, 1989. Zbl 0695.46033 MR 1093462

[20] A. Ya. Helemskii, Banach and locally convex algebras. The Clarendon Press, Oxford University Press, New York, 1993. Zbl 0785.46045 MR 1231796

[21] C. Kassel, Quantum groups. Graduate Texts in Mathematics, 155. Springer-Verlag, New York, 1995. Zbl 0808.17003 MR 1321145

[22] M. Khalkhali, Basic Noncommutative Geometry. EMS Series of Lectures in Mathematics. European Mathematical Society (EMS), Zurich, 2009. Zbl 1210.58006 MR 2567651

[23] M. Khalkhali, G. Landi, and W. D. van Suijlekom, Holomorphic structures on the quantum projective line. Int. Math. Res. Not. 4 (2011), no. 4, 851-884. Zbl 1219.58002 MR 2773332

[24] G. Köthe, Topological vector spaces II, Grundlehren der Mathematischen Wissenschaften [Fundamental Principles of Mathematical Science], 237. Springer-Verlag, New YorkBerlin, 1979. Zbl 0417.46001 MR 551623

[25] D. Luminet, A functional calculus for Banach PI-algebras. Pacific J. Math. 125 (1986), no. 1, 127-160. Zbl 0577.46050 MR 860755

[26] S. Majid, Cross products by braided groups and bosonization. J. Algebra 163 (1994), no. 1, 165-190. Zbl 0807.16036 MR 1257312

[27] S. Majid, Algebras and Hopf algebras in braided categories. Advances in Hopf algebras (Chicago, IL, 1992), 55-105, Lecture Notes in Pure and Appl. Math., 158, Dekker, New York, 1994. Zbl 0812.18004 MR 1289422 
[28] A. Mallios, Topological algebras. Selected topics. North-Holland Mathematics Studies, 124. Notas de Matemática [Mathematical Notes], 109. North-Holland Publishing Co., Amsterdam, 1986. Zbl 0597.46046 MR 857807

[29] Yu. I. Manin, Topics in noncommutative geometry. Princeton University Press, Princeton, NJ, 1991. Zbl 0724.17007 MR 1095783

[30] J. C. McConnell and J. C. Robson, Noncommutative Noetherian rings. Graduate Studies in Mathematics, 30. American Mathematical Society, Providence, RI, 2001. Zbl 0980.16019 MR 1811901

[31] E. A. Michael, Locally multiplicatively-convex topological algebras. Mem. Amer. Math. Soc., no. 11 (1952). Zbl 0047.35502 MR 51444

[32] B. Mitiagin, S. Rolewicz, and W. Żelazko, Entire functions in $B_{0}$-algebras. Studia Math. 21 (1961/1962), 291-306. Zbl 0111.31001 MR 144222

[33] C. Năstăsescu and F. Van Oystaeyen, Methods of graded rings. Lecture Notes in Mathematics, 1836. Springer-Verlag, Berlin, 2004. Zbl 1043.16017 MR 2046303

[34] A. Nica and R. Speicher, Lectures on the combinatorics of free probability. London Mathematical Society Lecture Note Series, 335. Cambridge University Press, Cambridge, 2006. Zbl 1133.60003 MR 2266879

[35] A. Pietsch, Nuclear locally convex spaces. Ergebnisse der Mathematik und ihrer Grenzgebiete, Band 66. Springer-Verlag, New York-Heidelberg, 1972. Zbl 0236.46002 MR 350360

[36] A. Yu. Pirkovskii, Stably flat completions of universal enveloping algebras, Dissertationes Math. (Rozprawy Math.) 441 (2006), 1-60. Zbl 1140.46031 MR 2283621

[37] A. Yu. Pirkovskii, Arens-Michael enveloping algebras and analytic smash products, Proc. Amer. Math. Soc. 134 (2006), no. 9, 2621-2631. Zbl 1100.46046 MR 2213741

[38] A. Yu. Pirkovskii, Arens-Michael envelopes, homological epimorphisms, and relatively quasi-free algebras (Russian), Tr. Mosk. Mat. Obs. 69 (2008), 34-125; English transl.: Trans. Moscow Math. Soc. 2008, 27-104. Zbl 1207.46064 MR 2549445

[39] A. Yu. Pirkovskii, Homological dimensions and Van den Bergh isomorphisms for nuclear Fréchet algebras. Izvestiya RAN: Ser. Mat. 76:4 (2012), 65-124 (in Russian). English transl.: Izvestiya: Mathematics, 76:4 (2012), 702-759. Zbl 1295.46056 MR 3013272

[40] A. Yu. Pirkovskii, Noncommutative analogues of Stein spaces of finite embedding dimension. Algebraic Methods in Functional Analysis, 135-153, Oper. Theory Adv. Appl., Vol. 233, Birkhäuser/Springer Basel AG, Basel, 2014. MR 3203988

[41] R. M. Range, Holomorphic functions and integral representations in several complex variables. Graduate Texts in Mathematics, 108. Springer-Verlag, New York, 1986. Zbl 0591.32002 MR 847923

[42] H. H. Schaefer, Topological vector spaces. The Macmillan Co., New York; CollierMacmillan Ltd., London, 1966. Zbl 0141.30503 MR 193469

[43] R. Street, Quantum groups. A path to current algebra. Australian Mathematical Society Lecture Series, 19. Cambridge University Press, Cambridge, 2007. Zbl 1117.16031 MR 2294803 
[44] M. E. Sweedler, Hopf algebras. Benjamin, New York, 1969. Zbl 0194.32901 MR 252485

[45] J. L. Taylor, Homology and cohomology for topological algebras, Adv. Math. 9 (1972), 137-182. Zbl 0271.46040 MR 328624

[46] J. L. Taylor, A general framework for a multi-operator functional calculus, Adv. Math. 9 (1972), 183-252. Zbl 0271.46041 MR 328625

[47] J. L. Taylor, Functions of several noncommuting variables. Bull. Amer. Math. Soc. 79 (1973), 1-34. Zbl 0257.46055 MR 315446

[48] J. C. Várilly, An introduction to noncommutative geometry. EMS Series of Lectures in Mathematics. European Mathematical Society (EMS), Zürich, 2006. Zbl 1097.58004 MR 2239597

[49] D. Vogt, The tensor algebra of the space s. Bull. Soc. Roy. Sci. Liège 70 (2001), no. 4-6, 435-440. Zbl 1030.46107 MR 1904066

[50] D. Vogt, The tensor algebra of power series spaces. Studia Math. 193 (2009), no. 2, 189-202. Zbl 1200.46040 MR 2515518

[51] K.-W. Wiegmann, Einbettungen komplexer Räume in Zahlenräume. Invent. Math. 1 (1966), 229-242. Zbl 0148.32003 MR 223604

Received 14 April, 2013

A. Yu. Pirkovskii, Faculty of Mathematics, National Research University

Higher School of Economics, 7 Vavilova, 117312 Moscow, Russia

E-mail: aupirkovskii@hse.ru; pirkosha@gmail.com 